\title{
New N-Heterocyclic Carbene Ligand and Its Application in Asymmetric Nickel-Catalyzed Aldehyde/Alkyne/Silane Reductive Couplings
}

\author{
Mani Raj Chaulagain, Grant Sormunen and John Montgomery* \\ 930 N. University Ave. Department of Chemistry, University of Michigan, Ann Arbor, MI 48109-1055 \\ jmontg@umich.edu
}

All reagents were used as received unless otherwise noted. Solvents were purified under nitrogen using a solvent purification system (Innovative Technology, inc., Model \# SPS400-3 and PS-400-3). Aldehydes were distilled prior to use. Ni(COD) 2 (Strem Chemicals, Inc., used as received), carbene precursors and potassium tert-butoxide were stored and weighed in an inert atmosphere glovebox. All reactions were conducted in flame-dried glassware under nitrogen. ${ }^{1} \mathrm{H},{ }^{13} \mathrm{C}$, and ${ }^{19} \mathrm{~F}$ spectra were obtained in $\mathrm{CDCl}_{3}$, unless otherwise noted on a Varian Mercury 300, Varian Inova 400 or $500 \mathrm{MHz}$ instrument. High resolution mass spectra (HRMS) were obtained on a VG (Micromass) 70-250-S magnetic sector mass spectrometer in the University of Michigan Mass Spectrometry facility. Chiral HPLC analyses were carried out using an Agilent Technologies 1100 series instrument and using chiralcel OJ column from Chiral Technologies Inc.

General procedure of the coupling of the chiral diamine and aryl bromide followed by cyclization.

$(1 R, 2 R)-(+)-1,2$-diphenylmethylenediamine (1.00 equiv), $\mathrm{Pd}_{2}\left(\mathrm{dba}_{3} \cdot \mathrm{CHCl}_{3}(0.05\right.$ equiv), BINAP (0.12 equiv) and sodium tert-butoxide (3.00 equiv) were weighed in a flask with a stir bar in an oxygen free glovebox and sealed with a rubber septum. A solution of the aryl bromide (2.4 equiv) in toluene $(3 \mathrm{~mL} / \mathrm{mmol})$ was added to the mixture and heated at $90{ }^{\circ} \mathrm{C}$ under nitrogen (unless otherwise noted). The crude reaction mixture was filtered 
through celite washed with methylene chloride, concentrated and purified by column chromatography ( $\mathrm{SiO}_{2}, 1: 10$ ethyl acetate/hexanes) to afford bis $N$-arylated products as soft pale yellow solids after the removal of solvents under high vacuum.

The bis-(arylamine) was dissolved in triethyl orthoformate (10.0 equiv based on the starting diamine) followed by the addition of ammonium tetrafluoroborate (1.0 equiv) and catalytic ( 1 drop) formic acid. The mixture was heated under nitrogen atmosphere at $120{ }^{\circ} \mathrm{C}$ for $12 \mathrm{~h}$. The crude reaction mixture was purified by column chromatography $\left(\mathrm{SiO}_{2}, 1: 10\right.$ methanol/ $\left.\mathrm{CH}_{2} \mathrm{Cl}_{2}\right)$ to afford 3,4-dihydroimidazolium tetrafluoroborate as hard foam pale yellow solids after removal of the solvents under high vacuum.

The compounds $\mathbf{1 a}$ and $\mathbf{1} \mathbf{b}$ were prepared by a literature procedure. ${ }^{1}$

\section{$1 R, 2 R-N^{1}, N^{2}$-Bis(2-cyclohexylphenyl)-1,2-diphenylethane-1,2-diamine}

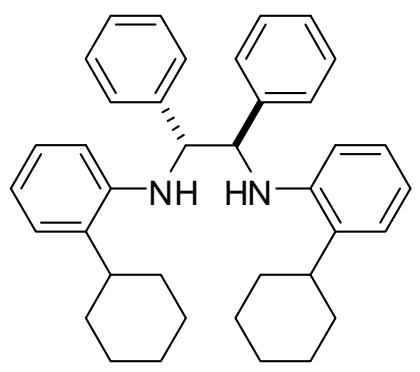

Using the general procedure, $(1 R, 2 R)-(+)-1,2$-diphenylmethylenediamine $(212 \mathrm{mg}, 1.00$ mmol), 1-bromo-2-cyclohexylbenzene (526 mg, $2.20 \mathrm{mmol}), \mathrm{Pd}_{2}(\mathrm{dba})_{3} \cdot \mathrm{CHCl}_{3}(52 \mathrm{mg}$, $0.05 \mathrm{mmol}$ ), racemic-BINAP (74 $\mathrm{mg}, 0.12 \mathrm{mmol})$ and sodium tert-butoxide $(288 \mathrm{mg}$, $3.00 \mathrm{mmol})$ were employed to afford $1 R, 2 R-N^{1}, N^{2}$-bis(2-cyclohexylphenyl)-1,2diphenylethane-1,2-diamine ( $482 \mathrm{mg}, 0.91 \mathrm{mmol}, 91 \%$ ) after column chromatography $\left(\mathrm{SiO}_{2}, 1: 20\right.$ ethyl acetate/hexanes) as a light orange solid. ${ }^{1} \mathrm{H} \mathrm{NMR}\left(400 \mathrm{MHz}, \mathrm{CDCl}_{3}\right) \delta$ $7.52(\mathrm{~d}, J=8.0 \mathrm{~Hz}, 4 \mathrm{H}) 7.40(\mathrm{t}, J=7.6 \mathrm{~Hz}, 4 \mathrm{H}) 7.28-7.34(\mathrm{~m}, 2 \mathrm{H}) 7.11(\mathrm{dd}, J=8.0,1.6$ 
$\mathrm{Hz}, 2 \mathrm{H}) 6.90(\mathrm{td}, J=8.0,1.6 \mathrm{~Hz}, 2 \mathrm{H}) 6.70(\mathrm{td}, J=7.6,1.2 \mathrm{~Hz}, 2 \mathrm{H}) 6.23(\mathrm{~d}, J=8.0 \mathrm{~Hz}$, 2H) $4.80(\mathrm{~s}, 2 \mathrm{H}) 4.66(\mathrm{~s}, 2 \mathrm{H}) 2.44-2.51(\mathrm{~m}, 2 \mathrm{H}) 2.15(\mathrm{~d}, J=11.2 \mathrm{H}, 2 \mathrm{H}) 1.67(\mathrm{~d}, J=11.2$ $\mathrm{Hz}, 2 \mathrm{H}) 1.82-2.05(\mathrm{~m}, 8 \mathrm{H}) 1.30-1.56(\mathrm{~m}, 12 \mathrm{H}) ;{ }^{13} \mathrm{C}\left(125 \mathrm{MHz}, \mathrm{CDCl}_{3}\right) \delta$ 143.6, 140.1, $132.2,129.0,127.8,126.8,126.4,125.3,117.8,112.3,63.9,38.7,32.9,32.6,27.5,27.4$, 26.5.

4R,5R-1,3-bis(2-cyclohexylphenyl)-4,5-dihydro-1H-imidazol-3-ium tetrafluoroborate (1d)

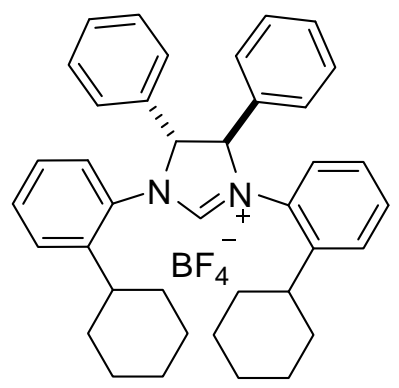

Following the general procedure, ammonium tetrafluoroborate $(105 \mathrm{mg}, 1.0 \mathrm{mmol})$, triethyl orthoformate $(1.60 \mathrm{~mL}, 10.0 \mathrm{mmol})$ and 1 drop formic acid along with the crude $N$-arylated product were employed to afford pure $4 R, 5 R$-1,3-bis(2-cyclohexylphenyl)-4,5diphenyl-4,5-dihydro-1 $\mathrm{H}$-imidazol-3-ium tetrafluoroborate $(520 \mathrm{mg}, 0.83 \mathrm{mmol}, 83 \%$ over 2 steps) after column chromatography $\left(\mathrm{SiO}_{2}, 1: 20\right.$ methanol/methylene chloride) as a light yellow solid foam. ${ }^{1} \mathrm{H}$ NMR $\left(400 \mathrm{MHz}, \mathrm{CDCl}_{3}\right) \delta 8.32(\mathrm{~s}, 1 \mathrm{H}) 7.54(\mathrm{~d}, J=7.6 \mathrm{~Hz}$, 2H) 7.11-7.46 (m, 16H) $5.67(\mathrm{~s}, 2 \mathrm{H}) 2.61-2.72(\mathrm{~m}, 2 \mathrm{H}) 1.65-1.98(\mathrm{~m}, 8 \mathrm{H})$ 1.28-1.55 (m, 8H) $1.20-1.28(\mathrm{~m}, 4 \mathrm{H}) ;{ }^{13} \mathrm{C}\left(125 \mathrm{MHz}, \mathrm{CDCl}_{3}\right) \delta 157.4,143.5,133.8,130.8,130.6$, 130.4, 129.7, 128.5, 128.1, 127.7, 127.6, 77.2, 39.5, 34.8, 34.0, 27.1, 27.0, 25.7; HRMS (EI) $\mathrm{m} / \mathrm{z}$ calculated for $[\mathrm{M}]^{+} 539.3426$, found 539.3430 . 


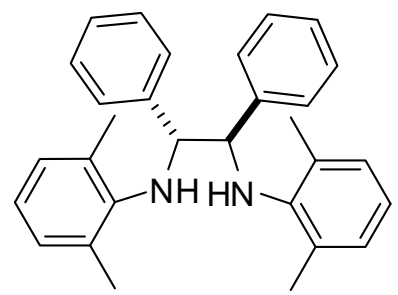

Using the general procedure, $(1 R, 2 R)-(+)-1,2-$ diphenylmethylenediamine $(106 \mathrm{mg}, 0.50$ mmol), 2-bromo-1,3-dimethylbenzene (222 mg, $1.20 \mathrm{mmol}), \mathrm{Pd}_{2}(\mathrm{dba})_{3} \cdot \mathrm{CHCl}_{3}(26 \mathrm{mg}$, $0.025 \mathrm{mmol}$ ), BINAP (37 mg, $0.06 \mathrm{mmol})$ and sodium tert-butoxide (144 mg, 3.00 mmol) were employed to afford $1 R, 2 R-N^{1}, N^{2}$-bis(2,6-dimethylphenyl)-1,2diphenylethane-1,2-diamine (180 $\mathrm{mg}, 0.43 \mathrm{mmol}, 86 \%$ ) after column chromatography $\left(\mathrm{SiO}_{2}, 1: 20\right.$ ethyl acetate/hexanes) as a light yellow solid. ${ }^{1} \mathrm{H} \mathrm{NMR}\left(400 \mathrm{MHz}, \mathrm{CDCl}_{3}\right) \delta$ 7.06-7.12 (m, 6H), 6.91-6.95 (m, 4H), $6.82(\mathrm{~d}, J=7.2 \mathrm{~Hz}, 4 \mathrm{H}), 6.65$ (t, $J=7.2 \mathrm{~Hz}, 2 \mathrm{H})$, $4.83(\mathrm{~s}, 2 \mathrm{H}), 4.02(\mathrm{bs}, 2 \mathrm{H}), 2.09(\mathrm{~s}, 12 \mathrm{H}) ;{ }^{13} \mathrm{C}\left(125 \mathrm{MHz}, \mathrm{CDCl}_{3}\right) \delta 144.2,140.3,129.1$, $128.3,128.0,127.7,127.2,120.9,66.1,19.5$

\section{R,5R-1,3-Bis(2,6-dimethylphenyl)-4,5-diphenyl-4,5-dihydro-1H-imidazol-3-ium tetrafluoroborate (1e)}

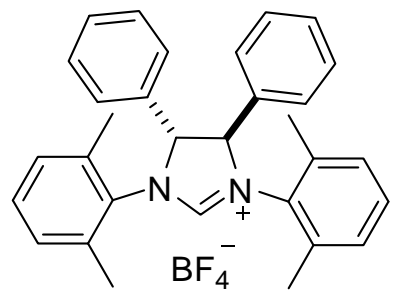

Following the general procedure, ammonium tetrafluoroborate (53 $\mathrm{mg}, 0.50 \mathrm{mmol})$, triethyl orthoformate $(0.83 \mathrm{~mL}, 10.00 \mathrm{mmol})$ and 1 drop formic acid along with the crude $N$-arylated product were employed to afford pure $4 R, 5 R$-1,3-bis(2,6-dimethylphenyl)-4,5- 
diphenyl-4,5-dihydro-1H-imidazol-3-ium tetrafluoroborate (1e) $(199 \mathrm{mg}, 0.39 \mathrm{mmol}, 77$ $\%$ over 2 steps) after column chromatography $\left(\mathrm{SiO}_{2}, 1: 10\right.$ methanol/methylene chloride) as light yellow solid foam after. ${ }^{1} \mathrm{H}$ NMR $\left(500 \mathrm{MHz}, \mathrm{CDCl}_{3}\right) \delta 8.63(\mathrm{~s}, 1 \mathrm{H}), 7.25-7.35(\mathrm{~m}$, 10H), 7.05-7.15 (m, 4H), $6.86(\mathrm{t}, J=4.0 \mathrm{~Hz}, 2 \mathrm{H}), 6.01(\mathrm{~s}, 2 \mathrm{H}), 2.66(\mathrm{~s}, 6 \mathrm{H}), 1.92(\mathrm{~s}, 6 \mathrm{H})$; ${ }^{13} \mathrm{C}\left(125 \mathrm{MHz}, \mathrm{CDCl}_{3}\right) \delta 157.9,136.5,134.7,131.1,131.0,130.8,130.3,129.4,128.8$, 72.7, 19.0, 18.2; HRMS (EI) m/z calculated for $[\mathrm{M}]^{+}$431.2487, found 431.2385.

\section{3,5-Dicyclohexyl toluene}

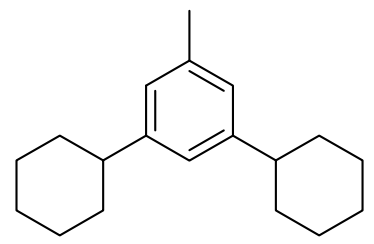

A solution of aluminum chloride (3.266 g, $24.5 \mathrm{mmol})$ in toluene $(1.078 \mathrm{~g}, 11.7 \mathrm{mmol})$ was cooled to $0{ }^{\circ} \mathrm{C}$. Cyclohexyl bromide $(4.006 \mathrm{~g}, 24.5 \mathrm{mmol})$ was added dropwise. The reaction was stirred for $45 \mathrm{~min}$, warming to rt. The reaction was then quenched with 15 $\mathrm{mL}$ ice, and diluted with $25 \mathrm{~mL}$ ether. The layers were separated, and the ether layer was washed twice with $10 \mathrm{~mL}$ water and $10 \mathrm{~mL}$ brine. The organic layer was then dried with $\mathrm{MgSO}_{4}$, filtered and concentrated to afford 3,5-dicyclohexyl toluene after column chromatography $\left(\mathrm{SiO}_{2}\right.$, hexanes) as a colorless oil $(2.344 \mathrm{~g}, 9.14 \mathrm{mmol}, 78 \%)$ which was previously reported. ${ }^{2}{ }^{1} \mathrm{H}$ NMR $\left(\mathrm{CDCl}_{3}, 400 \mathrm{MHz}\right) \delta 6.85(\mathrm{~s}, 3 \mathrm{H}), 2.43(\mathrm{~m}, 2 \mathrm{H}), 2.31$ (s, $3 \mathrm{H}), 1.80(\mathrm{~m}, 8 \mathrm{H}), 1.69(\mathrm{~m}, 2 \mathrm{H}), 1.35(\mathrm{~m}, 8 \mathrm{H}), 1.21(\mathrm{~m}, 2 \mathrm{H}) ;{ }^{13} \mathrm{C}$ NMR $\left(\mathrm{CDCl}_{3}, 100\right.$ MHz) $\delta 148.0,137.5,125.1,122.6,44.6,34.5,27.0,26.2,21.5$; IR (thin film, $\mathrm{KBr}, \mathrm{cm}^{-1}$ ) 3017, 2924, 2850, 1601, 1447, 847, 707. HRMS $\left(\mathrm{ES}^{+}\right)$calculated for $\mathrm{C}_{19} \mathrm{H}_{28}[\mathrm{M}]+$ 256.2191, found 256.2196. 


\section{2-Bromo-3,5-dicyclohexyl toluene}

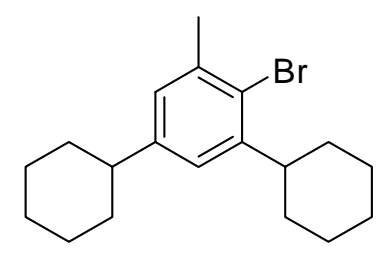

3,5-dicyclohexyltoluene $(0.6441 \mathrm{~g}, 2.512 \mathrm{mmol})$ was dissolved in $0.75 \mathrm{~mL}$ of chloroform and cooled to $0{ }^{\circ} \mathrm{C}$. Bromine $(0.4014 \mathrm{~g}, 2.512 \mathrm{mmol})$ was added slowly over $15 \mathrm{~min}$. The solution was allowed to warm to rt, then stirred for $16 \mathrm{~h}$. The reaction was diluted with $5 \mathrm{~mL}$ dichloromethane, which was washed with water. The water layer was extracted with dichloromethane twice and organics were combined and washed with 5 $\mathrm{mL} 10 \% \mathrm{NaOH}$, then brine, then dried with $\mathrm{MgSO}_{4}$, filtered and concentrated to afford a mixture of isomers (85:15), 2-bromo-3,5-dicyclohexyl toluene and 4-bromo-3,5dicyclohexyltoluene, as a colorless oil $(0.6002 \mathrm{~g}, 1.78 \mathrm{mmol}, 71 \%)$ after column chromatography $\left(\mathrm{SiO}_{2} /\right.$ hexanes). The minor isomer does not undergo $\mathrm{N}$-arylation in the following step based on recovery of starting material. ${ }^{1} \mathrm{H}$ NMR $\left(\mathrm{CDCl}_{3}, 400 \mathrm{MHz}\right) \delta$ 6.88-6.90 (m, $\left.2 \mathrm{H}_{\text {major }}\right), 6.87\left(\mathrm{~s}, 2 \mathrm{H}_{\text {minor }}\right), 3.00-3.09\left(\mathrm{~m}, 1 \mathrm{H}_{\text {major }}+2 \mathrm{H}_{\text {minor }}\right), 2.39-2.43(\mathrm{~m}$,

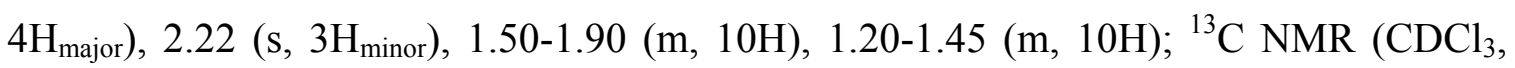
$100 \mathrm{MHz}) \delta 146.7,146.3,137.9,136.6$ (minor isomer), 126.8, 125.6 (minor isomer), 124.2, 123.2, 44.2, 44.0 (minor isomer), 43.7, 34.4, 33.4, 26.9, 26.8, 26.3, 26.1, 24.4; IR (thin film, $\mathrm{KBr}, \mathrm{cm}^{-1}$ ) 2924, 2850, 1461, 1447, 1017, 857; HRMS $\left(\mathrm{ES}^{+}\right)$calculated for $\mathrm{C}_{19} \mathrm{H}_{27} \mathrm{Br}[\mathrm{M}]^{+}$334.1296, found: 334.1287. 


\section{$1 R, 2 R-N^{1}, N^{2}$-bis(2,4-dicyclohexyl-6-methylphenyl)-1,2-diphenylethane-1,2-diamine}

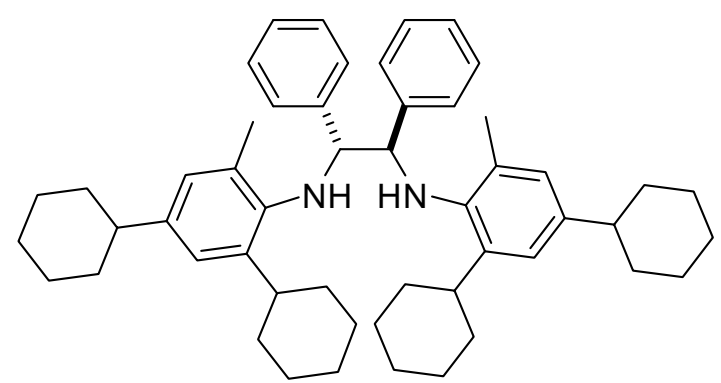

Following the general procedure, $(1 R, 2 R)-(+)-1,2$-diphenylmethylenediamine $(212 \mathrm{mg}$, $1.00 \mathrm{mmol}$ ), 2-bromo-3,5-dicyclohexyltoluene (801 $\mathrm{mg}, 2.40 \mathrm{mmol}), \mathrm{Pd}_{2}(\mathrm{dba})_{3} \cdot \mathrm{CHCl}_{3}$ (104 mg, $0.10 \mathrm{mmol})$, BINAP (148 mg, $0.24 \mathrm{mmol}$ ) and sodium tert-butoxide (288 mg, $3.00 \mathrm{mmol})$ were heated at $110{ }^{\circ} \mathrm{C}$ afford $1 R, 2 R-N^{1}, N^{2}$-bis(2,4-dicyclohexyl-6methylphenyl)-1,2-diphenylethane-1,2-diamine ( $598 \mathrm{mg}, 0.83 \mathrm{mmol}, 83 \%$ ) after column chromatography ( $\mathrm{SiO}_{2}, 1: 20$ ethyl acetate/hexanes) as a light orange solid. ${ }^{1} \mathrm{H}$ NMR (400 $\left.\mathrm{MHz}, \mathrm{CDCl}_{3}\right) \delta{ }^{1} \mathrm{H}_{\mathrm{NMR}}\left(400 \mathrm{MHz}, \mathrm{CDCl}_{3}\right) \delta$ 6.90-7.10 (m, 10H), $6.79(\mathrm{~s}, 2 \mathrm{H}), 6.68(\mathrm{~s}$, 2H), 4.50 (s, 2H), 4.04 (bs, 2H), 2.81-2.92 (m, 2H), 2.28-2.36 (m, 2H), $2.03(\mathrm{~s}, 6 \mathrm{H}), 1.58-$ $1.83(\mathrm{~m}, 20 \mathrm{H}), 1.10-1.39(\mathrm{~m}, 20 \mathrm{H}) ;{ }^{13} \mathrm{C}\left(125 \mathrm{MHz}, \mathrm{CDCl}_{3}\right) \delta 142.4,141.4,140.8,140.7$, $131.4,128.5,127.7,126.8,126.5,123.2,68.0,44.0,38.0,35.0,34.5,33.9,27.2,27.0$, 26.7, 26.3, 26.2, 19.5; HRMS (EI) $\mathrm{m} / \mathrm{z}$ calculated for $[\mathrm{M}+\mathrm{H}]^{+}$721.5461, found 721.5468 . 


\section{R,5R-1,3-bis(2,4-dicyclohexyl-6-methylphenyl)-4,5-diphenyl-4,5-dihydro-1H-}

imidazol-3-iumtetrafluoroborate (1f)

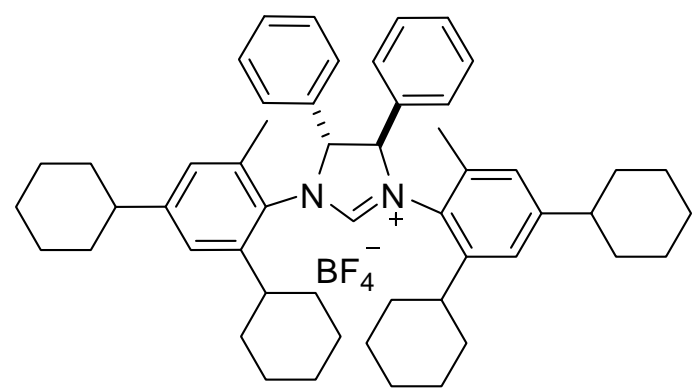

Following the general procedure, ammonium tetrafluoroborate $(105 \mathrm{mg}, 1.00 \mathrm{mmol})$, triethyl orthoformate $(1.66 \mathrm{~mL}, 10 \mathrm{mmol})$ and 1 drop formic acid along with the crude $\mathrm{N}$ arylated product were employed to afford pure 4R,5R-1,3-bis(2,4-dicyclohexyl-6methylphenyl)-4,5-diphenyl-4,5-dihydro-1 $\mathrm{H}$-imidazol-3-iumtetrafluoroborate (1f) (581 $\mathrm{mg}, 0.71 \mathrm{mmol}, 71 \%$ over 2 steps $)$ after column chromatography $\left(\mathrm{SiO}_{2}, 1: 20\right.$ methanol: methylene chloride) as light yellow foam. ${ }^{1} \mathrm{H}$ NMR $\left(400 \mathrm{MHz}, \mathrm{CDCl}_{3}\right) \delta 8.70(\mathrm{~s}, 0.17 \mathrm{H})$, $8.37(\mathrm{~s}, 0.33 \mathrm{H}), 8.00(\mathrm{~s}, 0.50 \mathrm{H})($ correspond to the three rotamers of a single proton), 6.70-7.26 (m, 14H), $6.11(\mathrm{~s}, 1.00 \mathrm{H}), 5.97(\mathrm{~d}, J=10.4 \mathrm{~Hz}, 0.33 \mathrm{H}), 5.85(\mathrm{~d}, J=10.4 \mathrm{~Hz}$, $0.33 \mathrm{H}), 5.78(\mathrm{~s}, 0.33 \mathrm{H})$ (correspond to three rotamers of two protons of the nitrogen heterocycle), 2.79-2.95 (m, 1H), 1.65-1.78 (m, 3H), 1.05-2.45 (m, 46H); ${ }^{13} \mathrm{C}(125 \mathrm{MHz}$, $\mathrm{CDCl}_{3}$ ) (due to existence of three rotamers, ${ }^{13} \mathrm{C}$ NMR spectrum appeared complex) $\delta$ 157.3, 156.7, 151.1, 150.7, 150.5, 145.9, 145.2, 144.1, 143.8, 134.2, 130.7, 129.8, 129.6, $129.4,129.3,129.2,128.9,128.8,127.9,126.9,124.1,75.0,72.7,72.2,44.3,44.2,44.2$, $40.4,40.3,39.6,35.9,34.1,34.0,33.9,32.6,31.9,27.0,26.8,26.7,26.6,26.5,25.9,25.7$, 19.6, 18.8, 18.6, 18.3; HRMS (EI) m/z calculated for $[\mathrm{M}]^{+}$731.5304, found 731.5306. 


\section{General procedure of the enantioselective aldehyde-alkyne coupling}

$\mathrm{Ni}(\mathrm{COD})_{2}(0.1$ equiv), ligand ( 0.1 equiv), and potassium tert-butoxide ( 0.1 equiv) were weighed in a flask inside the glove box and sealed with a rubber septum. The mixture was stirred in THF $(8 \mathrm{~mL} / \mathrm{mmol}$,) under inert atmosphere for $10 \mathrm{~min}$ at $\mathrm{rt}$. Triethylsilane (2.0 equiv) was added and stirred for 5 min at $0{ }^{\circ} \mathrm{C}$. Neat aldehyde (1.0 equiv) and the solution of alkyne (1.2 equiv) in THF $(2 \mathrm{~mL} / \mathrm{mmol})$ were added sequentially to the mixture at $\mathrm{rt}$. The reactions usually completes in $30 \mathrm{~min}$. The reaction was quenched by stirring in air for $15 \mathrm{~min}$ followed by concentration and purification by column chromatography.

\section{General procedure of TBAF deprotection}

To the solution of the TES-ether (1 equiv) in THF $(10 \mathrm{~mL} / \mathrm{mmol})$ was added tetrabutyl ammonium fluoride (TBAF) (2 equiv, $1 \mathrm{M}$ solution in $\mathrm{THF}$ ) and the mixture was stirred at $\mathrm{rt}$ for $2 \mathrm{~h}$. The reaction mixture was then diluted with ether $(10 \mathrm{~mL} / \mathrm{mmol})$ and washed with $\mathrm{NaHCO}_{3}(10 \mathrm{~mL} / \mathrm{mmol})$. The organic layer was then dried over $\mathrm{MgSO}_{4}$, filtered and concentrated. The crude product was purified by column chromatography.

\section{General procedure to make Mosher's ester from Mosher's acid chloride}

A solution of $(R)-(-)-\alpha$-methoxy- $\alpha$-trifluoromethylphenylacetyl chloride (R-MTPA-Cl) ( 2 equiv) (weighed in glove box in a vial) in $1 \mathrm{~mL} / \mathrm{mmol} \mathrm{CH}_{2} \mathrm{Cl}_{2}$, was added to a solution of alcohol (1 equiv) in $\mathrm{CH}_{2} \mathrm{Cl}_{2}(1 \mathrm{~mL} / \mathrm{mmol})$ at $0{ }^{\circ} \mathrm{C}$ under nitrogen followed by the addition of pyridine $(1 \mathrm{~mL} / \mathrm{mmol})$. The mixture was stirred until all the starting alcohol is consumed as judged by TLC analysis. The reaction mixture was filtered through a small silica plug and washed with $\mathrm{CH}_{2} \mathrm{Cl}_{2}$. 


\section{(R,E)-Triethyl(2-methyl-1,3-diphenylallyloxy)silane (entry 1)}

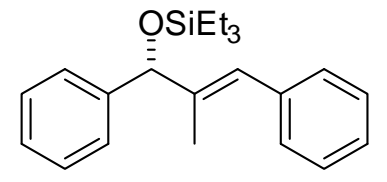

Following the general procedure, $\mathrm{Ni}(\mathrm{COD})_{2}(6 \mathrm{mg}, 0.02 \mathrm{mmol})$, ligand $(R, R), \mathbf{1 f}(16 \mathrm{mg}$, $0.02 \mathrm{mmol}), \mathrm{BuOK}(2.5 \mathrm{mg}, 0.02 \mathrm{mmol})$, triethylsilane $(64 \mu \mathrm{L}, 2.00 \mathrm{mmol}), 1$-phenyl-1propyne $(140 \mathrm{mg}, 1.25 \mathrm{mmol})$ and benzaldehyde $(100 \mu \mathrm{L}, 1.00 \mathrm{mmol})$ were employed to give (R,E)-triethyl(2-methyl-1,3-diphenylallyloxy)silane (331 mg, $0.98 \mathrm{mmol}, 98 \%$ yield, 10:1 regioselectivity), after column chromatography $\left(\mathrm{SiO}_{2}\right.$, hexanes) as a colorless oil. Spectral data of the compound were identical with the previously reported compound. ${ }^{3}$ Following the general procedure, silyl deprotection followed by HPLC analysis (using chiral column chiralcel OJ $1.0 \mathrm{~mL} / \mathrm{min}$ 99:1 hexanes/isopropanol) illustrated $78 \%$ ee (some variation in ee was observed ranging from 65-78\%) (see S30 for characteristic diagnostic peaks between 70-90 min. in chiral HPLC). Absolute stereochemistry was assigned by analogy.

\section{(R,E)-(2-Ethyl-1-phenylpent-2-enyloxy)triethylsilane (entry 2)}

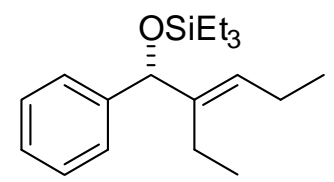

Following the general procedure, $\mathrm{Ni}(\mathrm{COD})_{2}(6 \mathrm{mg}, 0.02 \mathrm{mmol})$, ligand $(R, R), \mathbf{1 f}(16 \mathrm{mg}$, $0.02 \mathrm{mmol}$ ), $t \mathrm{BuOK}(3 \mathrm{mg}, 0.02 \mathrm{mmol})$, triethylsilane (64 $\mu \mathrm{L}, 0.4 \mathrm{mmol}), 3$-hexyne (20 $\mathrm{mg}, 0.24 \mathrm{mmol})$ and benzaldehyde $(20 \mu \mathrm{L}, 0.2 \mathrm{mmol})$ were employed to give $(R, E)-(2-$ ethyl-1-phenylpent-2-enyloxy)triethylsilane (50 mg, $0.16 \mathrm{mmol}, 82 \%$ yield), after column chromatography $\left(\mathrm{SiO}_{2}\right.$, hexanes) as a colorless oil. ${ }^{1} \mathrm{H} \mathrm{NMR}\left(400 \mathrm{MHz}, \mathrm{CDCl}_{3}\right) \delta$ 
7.28-7.36 (m, 2H), 7.22-7.28 (m, 2H), 7.14-7.20 (m, 1H), $5.48(\mathrm{t}, J=7.2 \mathrm{~Hz}, 1 \mathrm{H}), 5.07$ (s, 1H), 2.03 (dquint, $J=7.6,2.4 \mathrm{~Hz}, 2 \mathrm{H}), 1.86$ (dq, $J=7.6,3.2 \mathrm{~Hz}, 2 \mathrm{H}), 0.97$ (t, $J=7.6$ $\mathrm{Hz}, 3 \mathrm{H}), 0.88(\mathrm{t}, J=8.0 \mathrm{~Hz}, 9 \mathrm{H}), 0.70(\mathrm{t}, J=7.6 \mathrm{~Hz}, 3 \mathrm{H}), 0.56(\mathrm{q}, J=8.0 \mathrm{~Hz}, 6 \mathrm{H}) ;{ }^{13} \mathrm{C}$ $\left(125 \mathrm{MHz}, \mathrm{CDCl}_{3}\right) \delta 144.0,142.7,128.1,127.6,126.5,126.2,78.9,20.7,19.7,14.34$, 14.30, 6.8, 4.8; HRMS (EI) $\mathrm{m} / \mathrm{z}$ calculated for $\mathrm{M}^{+}$304.2222, found 304.2218. Following the general procedure, Mosher ester was synthesized (with R-MTPA-Cl) followed by ${ }^{1} \mathrm{H}$ and ${ }^{19} \mathrm{~F}$ NMR analysis which illustrated $70 \%$ ee (some variation in ee was observed ranging from $60-70 \%$ ) (see S32 for characteristic diagnostic peaks at -71.31 and -71.38 ppm for enantiopure and racemic spectra of $\left.{ }^{19} \mathrm{~F}-\mathrm{NMR}\right)$.

\section{(S,E)-(2,4-Dimethyl-1-phenylpent-1-en-3-yloxy)triethylsilane (entry 3)}

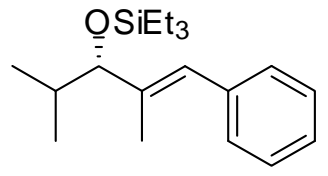

Following the general procedure, $\mathrm{Ni}(\mathrm{COD})_{2}(6 \mathrm{mg}, 0.02 \mathrm{mmol})$, ligand $(R, R), \mathbf{1 f}(16 \mathrm{mg}$, $0.02 \mathrm{mmol}$ ), $t \mathrm{BuOK}(2.5 \mathrm{mg}, 0.02 \mathrm{mmol})$, triethylsilane ( $64 \mu \mathrm{L}, 0.4 \mathrm{mmol}), 1-$ phenyl-1propyne (28 $\mathrm{mg}, 0.24 \mathrm{mmol})$ and isobutyraldehyde $(18 \mu \mathrm{L}, 0.2 \mathrm{mmol})$ were employed to give (S,E)-(2,4-dimethyl-1-phenylpent-1-en-3-yloxy)triethylsilane (52 mg, $0.17 \mathrm{mmol}, 86$ $\%$ yield), after column chromatography $\left(\mathrm{SiO}_{2}\right.$, hexanes) as a colorless oil. ${ }^{1} \mathrm{H}$ NMR (400 $\left.\mathrm{MHz}, \mathrm{CDCl}_{3}\right) \delta$ 7.27-7.32 (m, 2H), 7.21-7.25 (m, 2H), 7.15-7.20 (m, 1H), $6.33(\mathrm{~s}, 1 \mathrm{H})$, $3.66(\mathrm{~d}, J=8.0 \mathrm{~Hz}, 1 \mathrm{H}), 1.77(\mathrm{~d}, J=1.2 \mathrm{~Hz}, 3 \mathrm{H}), 1.72-1.82(\mathrm{~m}, 1 \mathrm{H}), 0.95(\mathrm{~d}, J=6.8 \mathrm{~Hz}$, $3 \mathrm{H}), 0.93(\mathrm{t}, J=8.0 \mathrm{~Hz}, 9 \mathrm{H}), 0.79(\mathrm{~d}, J=6.8 \mathrm{~Hz}, 3 \mathrm{H}), 0.58(\mathrm{q}, J=0.8 \mathrm{~Hz}, 6 \mathrm{H}) ;{ }^{13} \mathrm{C}(125$ $\left.\mathrm{MHz}, \mathrm{CDCl}_{3}\right) \delta 140.3,137.9,128.8,128.0,126.2,126.1,84.9,32.2,19.4,18.9,13.1,6.9$, 4.9; IR (Thin film, KBr, $\mathrm{cm}^{-1}$ ) 3021, 2954, 2875, 1454, 1064, 1009, 746, 698; HRMS 
(EI) $\mathrm{m} / \mathrm{z}$ calculated for $[\mathrm{M}+\mathrm{H}]^{+}$305.2301, found 305.2312. Following the general procedure, Mosher ester was synthesized (with $R$ - MTPA-Cl) followed by ${ }^{19} \mathrm{~F}$ NMR analysis which illustrated $70 \%$ ee (see S34 for characteristic diagnostic peaks at -70.92 and -71.32 ppm for enantiopure and racemic spectra of ${ }^{19} \mathrm{~F}-\mathrm{NMR}$ ).

(S,E)-(4-Ethylidene-2-methyl-7-phenylheptan-3-yloxy)triethylsilane and $S, E-(2,4-$

\section{Dimethyl-8-phenyl-oct-4-en-3-yloxy)triethylsilane (entry 4)}

Following the general procedure, $\mathrm{Ni}(\mathrm{COD})_{2}(6 \mathrm{mg}, 0.02 \mathrm{mmol})$, ligand $(R, R), \mathbf{1 f}(16 \mathrm{mg}$, $0.02 \mathrm{mmol}), t \mathrm{BuOK}(2.5 \mathrm{mg}, 0.02 \mathrm{mmol})$, triethylsilane $(64 \mu \mathrm{L}, 0.4 \mathrm{mmol})$, hex-4ynylbenzene $(38 \mathrm{mg}, 0.24 \mathrm{mmol})$ and isobutyraldehyde $(18 \mu \mathrm{L}, 0.2 \mathrm{mmol})$ were employed to give a mixture of regioisomers (S,E)-triethyl(4-ethylidene-2-methyl-7phenylheptan-3-yloxy)silane and S,E-(2,4-dimethyl-8-phenyl-oct-4-en-3yloxy)triethylsilane (60 $\mathrm{mg}, 0.17 \mathrm{mmol}, 86 \%$ yield, $3: 1$ regioselectivity), after column chromatography ( $\mathrm{SiO}_{2}$, hexanes) as a colorless oil.

(S,E)-(4-Ethylidene-2-methyl-7-phenylheptan-3-yloxy)triethylsilane

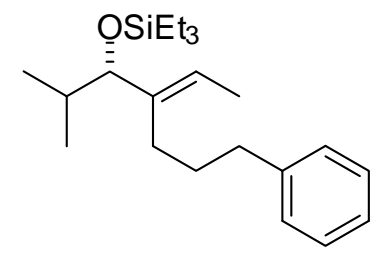

${ }^{1} \mathrm{H}$ NMR $\left(400 \mathrm{MHz}, \mathrm{CDCl}_{3}\right) \delta$ 7.21-7.28 (m, 2H), 7.12- $7.18(\mathrm{~m}, 3 \mathrm{H}), 5.31(\mathrm{q}, J=6.8 \mathrm{~Hz}$, $1 \mathrm{H}), 3.55(\mathrm{~d}, J=7.6 \mathrm{~Hz}, 1 \mathrm{H}), 2.60(\mathrm{t}, J=8.0 \mathrm{~Hz}, 2 \mathrm{H}), 2.00(\mathrm{t}, J=8.0 \mathrm{~Hz}, 2 \mathrm{H}), 1.58-1.74$ (m, 3H), $1.53(\mathrm{~d}, J=6.8 \mathrm{~Hz}, 3 \mathrm{H}), 0.90(\mathrm{t}, J=8.0 \mathrm{~Hz}, 9 \mathrm{H}), 0.84(\mathrm{~d}, J=6.4 \mathrm{~Hz}, 3 \mathrm{H}), 0.73$ $(\mathrm{d}, J=6.4 \mathrm{~Hz}, 3 \mathrm{H}), 0.53(\mathrm{q}, J=8.0 \mathrm{~Hz}, 6 \mathrm{H}) ;{ }^{13} \mathrm{C}\left(125 \mathrm{MHz}, \mathrm{CDCl}_{3}\right) \delta 142.5,141.3$, 128.3, 128.2, 125.6, 121.5, 83.7, 36.7, 32.1, 31.0, 26.7, 19.9, 18.4, 12.9, 6.9, 4.9; IR (Thin 
film, $\mathrm{KBr}, \mathrm{cm}^{-1}$ ) 3022, 2953, 2875, 1054, 1006, 740; HRMS (EI) $\mathrm{m} / \mathrm{z}$ calculated for $\left[\mathrm{M}^{+}+\mathrm{Na}\right]$ 369.2590, found 369.2584. Following the general procedure, silyl deprotection followed by HPLC analysis (using chiral column chiralcel OJ $1 \mathrm{~mL} / \mathrm{min}$ 99:1 hexanes/isopropanol) illustrated $75 \%$ ee (see S36 for characteristic diagnostic peaks between 39-50 min. in HPLC traces). Absolute stereochemistry was assigned by analogy.

\section{S,E-(2,4-Dimethyl-8-phenyl-oct-4-en-3-yloxy)triethylsilane}

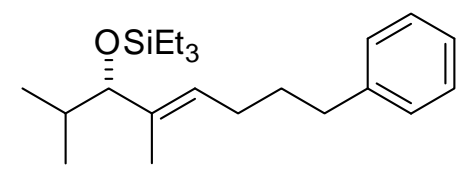

${ }^{1} \mathrm{H}$ NMR (400 MHz, $\left.\mathrm{CDCl}_{3}\right) \delta$ 7.21-7.28 (m, 2H), 7.12-7.18 (m, 3H), $5.26(\mathrm{t}, J=7.2 \mathrm{~Hz}$, $1 \mathrm{H}), 3.47(\mathrm{~d}, J=8.0 \mathrm{~Hz}, 1 \mathrm{H}), 2.59(\mathrm{t}, J=8.0 \mathrm{~Hz}, 2 \mathrm{H}), 2.02(\mathrm{q}, J=7.2 \mathrm{~Hz}, 2 \mathrm{H}), 1.60-1.72$ (m, 3H), 1.50 (s, 3H), 0.91 (t, $J=8.0 \mathrm{~Hz}, 9 \mathrm{H}), 0.91(\mathrm{~d}, J=6.8 \mathrm{~Hz}, 3 \mathrm{H}), 0.69$ (d, $J=6.8$ $\mathrm{Hz}, 3 \mathrm{H}), 0.54(\mathrm{q}, J=8.0 \mathrm{~Hz}, 6 \mathrm{H}) ;{ }^{13} \mathrm{C}\left(125 \mathrm{MHz}, \mathrm{CDCl}_{3}\right) \delta 142.6,137.5,128.3,128.2$, 126.4, 125.6, 85.0, 35.6, 31.9, 31.3, 27.0, 19.3, 19.2, 11.1, 6.9, 4.8.

(R,E)-(1-Cyclohexyl-2-ethylpent-2-enyloxy)triethylsilane (entry 5)

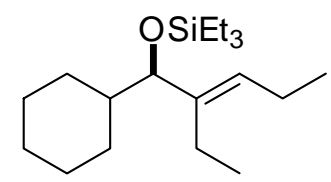

Following the general procedure, $\mathrm{Ni}(\mathrm{COD})_{2}(6 \mathrm{mg}, 0.02 \mathrm{mmol})$, ligand $(S, S), \mathbf{1 f}(16 \mathrm{mg}$, $0.02 \mathrm{mmol}$ ), $t \mathrm{BuOK}(3 \mathrm{mg}, 0.02 \mathrm{mmol}$ ), triethylsilane (64 $\mu \mathrm{L}, 0.40 \mathrm{mmol}), 3$-hexyne (20 $\mathrm{mg}, 0.24 \mathrm{mmol})$ and cyclohexanecarboxaldehyde $(24 \mu \mathrm{L}, 0.2 \mathrm{mmol})$ were employed to give (R,E)-(1-cyclohexyl-2-ethylpent-2-enyloxy)triethylsilane $(52 \mathrm{mg}, 0.17 \mathrm{mmol} 84 \%$ yield), after column chromatography ( $\mathrm{SiO}_{2}$, hexanes) as a colorless oil. ${ }^{1} \mathrm{H}$ NMR (400 $\left.\mathrm{MHz}, \mathrm{CDCl}_{3}\right) \delta 5.05(\mathrm{t}, J=7.2 \mathrm{~Hz}, 1 \mathrm{H}), 3.46(\mathrm{~d}, J=8.0 \mathrm{~Hz}, 1 \mathrm{H}), 1.79-1.96(\mathrm{~m}, 5 \mathrm{H})$, 
1.46-1.64 (m, 3H), 1.32-1.40 (m, 1H), 1.18-1.28 (m, 1H), 0.95-1.10 (m, 3H), 0.78-0.88 (m, 15H), 0.60-0.76 (m, 2H), $0.43(\mathrm{q}, J=8.0 \mathrm{~Hz}, 6 \mathrm{H}) ;{ }^{13} \mathrm{C}\left(125 \mathrm{MHz}, \mathrm{CDCl}_{3}\right) \delta$ 140.7, $129.1,83.2,41.5,30.1,29.0,26.6,26.15,26.13,20.5,19.5,14.3,14.1,6.8,4.8$; HRMS (EI) $\mathrm{m} / \mathrm{z}$ calculated for $\mathrm{M}^{+} 310.2691$, found 310.2677 . Following the general procedure, Mosher ester was synthesized (with $R$-MTPA-Cl) followed by ${ }^{19} \mathrm{~F}$ NMR analysis which illustrated $85 \%$ ee (see S38 for characteristic diagnostic peaks at -70.96 and $-71.38 \mathrm{ppm}$ for enantiopure and racemic spectra of ${ }^{19}$ F-NMR).

\section{(S,E)-(4-Ethyl-1-phenylhept-4-en-3-yloxy)triethylsilane (entry 6)}

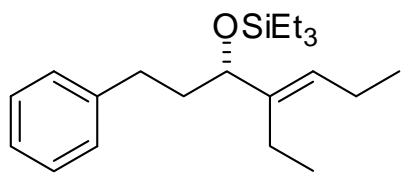

Following the general procedure, $\mathrm{Ni}(\mathrm{COD})_{2}(2.7 \mathrm{mg}, 0.01 \mathrm{mmol})$, ligand $(R, R), \mathbf{1 f}(8.2$ $\mathrm{mg}, 0.01 \mathrm{mmol}), \mathrm{BuOK}(1.1 \mathrm{mg}, 0.01 \mathrm{mmol})$, triethylsilane (32 $\mu \mathrm{L}, 0.2 \mathrm{mmol})$, 3-hexyne (17.3 $\mathrm{mg}, 0.21 \mathrm{mmol}$ ) and 3-phenylpropanal $(13.4 \mathrm{mg}, 0.1 \mathrm{mmol})$ were employed to give (S,E)-triethyl(4-ethyl-1-phenylhent-4-en-3-yloxy)silane (26 mg, $0.08 \mathrm{mmol}, 75 \%$ yield),

after column chromatography $\left(\mathrm{SiO}_{2}\right.$, hexanes) as a colorless oil. ${ }^{1} \mathrm{H} \mathrm{NMR}\left(\mathrm{CDCl}_{3}, 400\right.$ MHz) $\delta$ 7.21-7.23 (m, 2H), 7.13-7.15 (m, 3H), $5.27(\mathrm{t}, J=7.2 \mathrm{~Hz}, 1 \mathrm{H}), 3.99(\mathrm{t}, J=6.4$ Hz, 1H), 2.61 (dt, $J=13.6,8.2 \mathrm{~Hz}, 1 \mathrm{H}), 2.49$ (dt, $J=14.4,8.0 \mathrm{~Hz}, 1 \mathrm{H}), 1.98-2.03$ (m, 4H), $1.78(\mathrm{q}, J=7.8 \mathrm{~Hz}, 2 \mathrm{H}), 0.88-0.99(\mathrm{~m}, 15 \mathrm{H}), 0.54(\mathrm{q}, J=8.0 \mathrm{~Hz}, 6 \mathrm{H}) ;{ }^{13} \mathrm{C}$ NMR $\left(\mathrm{CDCl}_{3}, 100 \mathrm{MHz}\right) \delta 142.7,141.9,128.3,128.2,128.1,125.5,77.2,38.6,29.7,20.6$, 19.7, 14.6, 14.3, 6.9, 4.9.; IR (thin film, $\mathrm{KBr}, \mathrm{cm}^{-1}$ ) 3027, 2959, 2875, 1456, 1238, 1073, 1004, 742, 698; HRMS (ES ${ }^{+}$) calculated for $\mathrm{C}_{19} \mathrm{H}_{27} \mathrm{Br}[\mathrm{M}]^{+}$334.1296, found: 334.1287. Following the general procedure, Mosher ester was synthesized with S-Mosher's acid 
followed by ${ }^{19} \mathrm{~F}$ NMR analysis which illustrated $78 \%$ ee (see S40 for characteristic diagnostic peaks at -70.05 and $-71.36 \mathrm{ppm}$ for enantioenriched and racemic spectra of ${ }^{19}$ F-NMR).

\section{(S,E)-(1-Cyclohexyl-2-methyl-3-phenylallyloxy)triethylsilane (entry 7)}

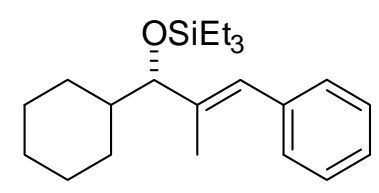

Following the general procedure, $\mathrm{Ni}(\mathrm{COD})_{2}(6 \mathrm{mg}, 0.02 \mathrm{mmol})$, ligand $(R, R), \mathbf{1 f}(16 \mathrm{mg}$, $0.02 \mathrm{mmol}), \mathrm{BuOK}(3 \mathrm{mg}, 0.02 \mathrm{mmol})$, triethylsilane $(64 \mu \mathrm{L}, 0.4 \mathrm{mmol}), 1-$ phenyl-1propyne $(28 \mathrm{mg}, 0.24 \mathrm{mmol})$ and cyclohexanecarboxaldehyde $(24 \mu \mathrm{L}, 0.2 \mathrm{mmol})$ were employed to give (S,E)-(1-cyclohexyl-2-methyl-3-phenylallyloxy)triethylsilane (54 mg, 0.157 mmol, $78 \%$ yield, 91:9 regioselectivity), after column chromatography ( $\mathrm{SiO}_{2}$, hexanes) as a colorless oil. ${ }^{1} \mathrm{H}$ NMR $\left(500 \mathrm{MHz}, \mathrm{CDCl}_{3}\right) \delta$ 7.32-7.37 (m, 2H), 7.25-7.31 (m, 2H), 7.19-7.24 (m, 1H), $6.34(\mathrm{~s}, 1 \mathrm{H}), 3.75(\mathrm{~d}, J=8.0 \mathrm{~Hz}, 1 \mathrm{H}), 2.03-2.10(\mathrm{~m}, 1 \mathrm{H})$, $1.82(\mathrm{~d}, J=1.5 \mathrm{~Hz}, 3 \mathrm{H}), 1.64-1.80(\mathrm{~m}, 3 \mathrm{H}), 1.46-1.50(\mathrm{~m}, 2 \mathrm{H}), 1.10-1.30(\mathrm{~m}, 3 \mathrm{H}), 0.97$ $(\mathrm{t}, J=8.0 \mathrm{~Hz}, 9 \mathrm{H}), 0.88-0.95(\mathrm{~m}, 2 \mathrm{H}), 0.63(\mathrm{q}, J=8.0 \mathrm{~Hz}, 6 \mathrm{H}) ;{ }^{13} \mathrm{C}\left(125 \mathrm{MHz}, \mathrm{CDCl}_{3}\right) \delta$ $140.1,137.9,128.8,128.0,126.4,126.1,84.1,41.7,29.8,29.6,26.4,26.3,26.2,13.1,6.9$, 4.9; HRMS (EI) $m / z$ calculated for $\mathrm{C}_{22} \mathrm{H}_{36} \mathrm{OSiNa}[\mathrm{M}+\mathrm{Na}]^{+}$367.2433, found 367.2417. Following the general procedure, Mosher ester was synthesized (with R-MTPA-Cl) followed by ${ }^{1} \mathrm{H}$ NMR analysis which illustrated $81 \%$ ee (see $\mathrm{S} 42$ for characteristic diagnostic peaks at -70.96 and -71.37 ppm for enantiopure and racemic spectra of ${ }^{19} \mathrm{~F}$ NMR). 


\section{(S,E)-(1-Cyclohexylnon-2-enyloxy)triethylsilane (entry 8)}

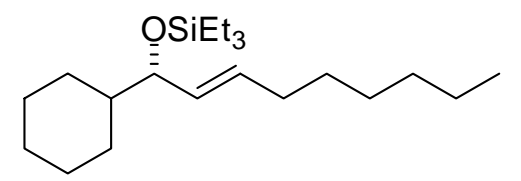

Following the general procedure, $\mathrm{Ni}(\mathrm{COD})_{2}(6 \mathrm{mg}, 0.02 \mathrm{mmol})$, ligand $(R, R), \mathbf{1 f}(16 \mathrm{mg}$, $0.02 \mathrm{mmol}), \mathrm{BuOK}(3 \mathrm{mg}, 0.02 \mathrm{mmol})$, triethylsilane (64 $\mu \mathrm{L}, 0.40 \mathrm{mmol}), 1$-octyne (26 $\mathrm{mg}, 0.24 \mathrm{mmol})$ and cyclohexanecarboxaldehyde $(24 \mu \mathrm{L}, 0.20 \mathrm{mmol})$ were employed to give (S,E)-(1-cyclohexylnon-2-enyloxy)triethylsilane (43 mg, $0.13 \mathrm{mmol}, 64 \%$ yield), after column chromatography ( $\mathrm{SiO}_{2}$, hexanes) as a colorless oil. ${ }^{1} \mathrm{H}$ NMR $(500 \mathrm{MHz}$, $\left.\mathrm{CDCl}_{3}\right) \delta 5.47(\mathrm{dt}, J=15.5,6.5 \mathrm{~Hz}, 1 \mathrm{H}), 5.37(\mathrm{dd}, J=15.0,8.0 \mathrm{~Hz}, 1 \mathrm{H}), 3.70(\mathrm{t}, J=7.5$ $\mathrm{Hz}, 1 \mathrm{H}), 2.02(\mathrm{q}, J=7.0 \mathrm{~Hz}, 2 \mathrm{H}), 1.84-1.90(\mathrm{~m}, 1 \mathrm{H}), 1.68-1.77(\mathrm{~m}, 2 \mathrm{H}), 1.61-1.68(\mathrm{~m}$, 2H), 1.01-1.42 (m, 12H), $0.95(\mathrm{t}, J=7.5 \mathrm{~Hz}, 9 \mathrm{H}), 0.86-0.93(\mathrm{~m}, 5 \mathrm{H}), 0.58(\mathrm{~m}, 6 \mathrm{H}) ;{ }^{13} \mathrm{C}$ $\left(125 \mathrm{MHz}, \mathrm{CDCl}_{3}\right) \delta 132.2,131.4,78.5,44.4,32.1,31.6,29.2,29.0,28.9,28.8,26.6$, 26.2, 26.1, 22.6, 14.0, 6.8, 5.0; HRMS (EI) $\mathrm{m} / \mathrm{z}$ calculated for $\mathrm{M}^{+}$338.3005, found 338.2992. Following general procedure, Mosher's ester was synthesized (with R-MTPACl) followed by ${ }^{19} \mathrm{~F}$ NMR analysis which illustrated $65 \%$ ee (see S44 for characteristic diagnostic peaks at -71.33 and -71.53 ppm for enantiopure and racemic spectra of ${ }^{19} \mathrm{~F}$ NMR).

\section{(S,E)-(2-Methyl-1-phenylnon-1-en-3-yloxy)triethylsilane (entry 9)}

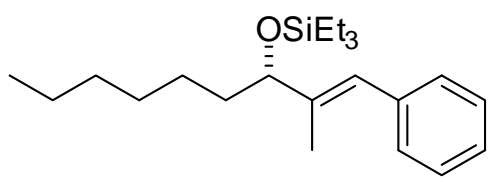

Following the general procedure, $\mathrm{Ni}(\mathrm{COD})_{2}(6 \mathrm{mg}, 0.02 \mathrm{mmol})$, ligand $(R, R) \mathbf{1 f}(16 \mathrm{mg}$, $0.02 \mathrm{mmol}), \mathrm{BuOK}(3 \mathrm{mg}, 0.02 \mathrm{mmol})$, triethylsilane $(64 \mu \mathrm{L}, 0.4 \mathrm{mmol}), 1-$ phenyl-1- 
propyne ( $28 \mathrm{mg}, 0.24 \mathrm{mmol})$ and heptaldehyde ( $28 \mu \mathrm{L}, 0.2 \mathrm{mmol})$ were employed to give (S,E)-triethyl(2-methyl-1-phenylnon-1-en-3-yloxy)silane (49 mg, $0.14 \mathrm{mmol}, 70 \%$ yield, 10:1 regioselectivity), after column chromatography ( $\mathrm{SiO}_{2}$, hexanes) as a colorless oil. Spectral data of the compound were identical with the previously reported compound. ${ }^{3}$ Following the general procedure, Mosher ester was synthesized (with $R$-MTPA-Cl) followed by ${ }^{1} \mathrm{H}$ NMR analysis which illustrated $73 \%$ ee (see S46 for characteristic diagnostic peaks at 6.56 and $6.48 \mathrm{ppm}$ for enantiopure and racemic spectra of ${ }^{1} \mathrm{H}$ NMR).

(S,E)-5-(Cyclohexyl(triethylsiloxy)methylhex-5-en-1-ol (entry 10)

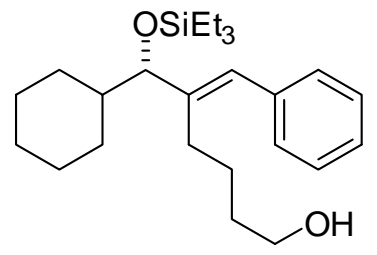

Following the general procedure, $\mathrm{Ni}(\mathrm{COD})_{2}(6 \mathrm{mg}, 0.02 \mathrm{mmol})$, ligand $(R, R) \mathbf{1 f}(16 \mathrm{mg}$, $0.02 \mathrm{mmol}$ ), $t \mathrm{BuOK}(3 \mathrm{mg}, 0.02 \mathrm{mmol}$ ), triethylsilane (64 $\mu \mathrm{L}, 0.4 \mathrm{mmol})$, 6-phenylhex-5yn-1-ol (42 mg, $0.24 \mathrm{mmol})$ and cyclohexanecarboxaldehyde $(24 \mu \mathrm{L}, 0.2 \mathrm{mmol})$ were employed to give (S,E)-5-(cyclohexyl(triethylsiloxy)methylhex-5-en-1-ol (79 mg, 0.196 mmol, $99 \%$ yield, 90:10 regioselectivity), after column chromatography ( $\mathrm{SiO}_{2}$, hexanes) as a colorless oil. ${ }^{1} \mathrm{H}$ NMR $\left(400 \mathrm{MHz}, \mathrm{CDCl}_{3}\right) \delta$ 7.11-7.32 (m, 5H), $6.34(\mathrm{~s}, 0.9 \mathrm{H}$, major isomer), 5.57 (t, $J=7.6 \mathrm{~Hz}, 0.10 \mathrm{H}$, minor isomer), $4.00(\mathrm{~d}, J=5.2 \mathrm{~Hz}, 0.10 \mathrm{H}$, minor isomer), $3.81(\mathrm{~d}, J=6.8 \mathrm{~Hz}, 0.90 \mathrm{H}$, major isomer), $3.56(\mathrm{bs}, 2 \mathrm{H}), 2.20-2.30(\mathrm{~m}, 1 \mathrm{H})$, 2.08-2.18 (m, 1H), 1.30-1.96 (m, 11H), 1.02-1.30 (m, 4H), $0.93(\mathrm{t}, J=8.0 \mathrm{~Hz}, 9 \mathrm{H}), 0.64$ (q, $J=8.0,6 \mathrm{H}) ;{ }^{13} \mathrm{C}\left(125 \mathrm{MHz}, \mathrm{CDCl}_{3}\right)$ (major isomer) $\delta 143.6,138.1,128.4,128.2$, 
$126.8,126.2,82.4,62.4,41.9,33.1,30.4,28.3,27.6,26.5,26.3,26.2,25.0,7.0,4.9$;

HRMS (EI) $\mathrm{m} / \mathrm{z}$ calculated for $[\mathrm{M}+\mathrm{Na}]^{+} 425.2852$, found 425.2850 .

(S,E)-(2-Benzylidene-1-cyclohexyl-6-methoxyhexyloxy)triethylsilane

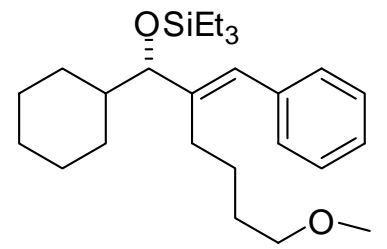

To a suspension of $\mathrm{NaH}(6 \mathrm{mg}, 0.22 \mathrm{mmol})$ in $0.5 \mathrm{~mL}$ DMF under nitrogen, a solution of the alcohol $(80 \mathrm{mg}, 0.20 \mathrm{mmol})$ in $\mathrm{DMF}(1 \mathrm{~mL})$ was added at $0{ }^{\circ} \mathrm{C}$ and stirred for $30 \mathrm{~min}$. MeI $(14 \mu \mathrm{L}, 0.21 \mathrm{mmol})$ was the added to the reaction mixture which gave the product (S,E)-(2-benzylidene-1-cyclohexyl-6-methoxyhexyloxy)triethylsilane $\quad\left(\begin{array}{l}75 \\ \mathrm{mg},\end{array} 0.18\right.$ mmol, $89 \%$ yield), after column chromatography $\left(\mathrm{SiO}_{2}\right.$, hexanes:ethyl acetate/20:1) as a colorless oil ${ }^{1} \mathrm{H}$ NMR $\left(300 \mathrm{MHz}, \mathrm{CDCl}_{3}\right) \delta$ 7.14-7.36 (m, 5H), $6.38(\mathrm{~s}, 0.9 \mathrm{H}$, major isomer), 5.60 (t, $J=7.5 \mathrm{~Hz}, 0.10 \mathrm{H}$, minor isomer), $4.03(\mathrm{~d}, J=4.8 \mathrm{~Hz}, 0.1 \mathrm{H}$, minor isomer), $3.86(\mathrm{~d}, J=6.3 \mathrm{~Hz}, 0.9 \mathrm{H}$, major isomer), $3.27-3.36(\mathrm{~m}, 5 \mathrm{H}), 1.02-2.40(\mathrm{~m}, 17 \mathrm{H})$, $0.97(\mathrm{t}, J=7.5 \mathrm{~Hz}, 9 \mathrm{H}), 0.64(\mathrm{t}, J=7.5 \mathrm{~Hz}, 6 \mathrm{H}) ;{ }^{13} \mathrm{C}\left(125 \mathrm{MHz}, \mathrm{CDCl}_{3}\right)$ (major isomer) $\delta$ $143.7,138.1,128.5,128.1,126.7,126.1,82.2,72.3,58.5,41.9,30.5,30.1,28.1,28.0$, 26.6, 26.4, 26.3, 25.4, 7.0, 5.0. Following the general procedure, Mosher ester was synthesized (with $R$-MTPA-Cl) followed by ${ }^{1} \mathrm{H}$ NMR analysis which illustrated $79 \%$ ee (see S48 for characteristic diagnostic peaks at -70.72 and -71.17 ppm for enantiopure and racemic spectra of $\left.{ }^{19} \mathrm{~F} N \mathrm{NM}\right)$.

\section{(S,E)-(1-Cyclohexyl-2-ethylideneheptyloxy)triethylsilane (entry 11)}




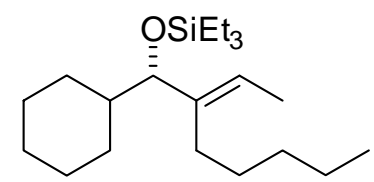

Following the general procedure, $\mathrm{Ni}(\mathrm{COD})_{2}(6 \mathrm{mg}, 0.02 \mathrm{mmol})$, ligand $(R, R) \mathbf{1 f}(16 \mathrm{mg}$, $0.02 \mathrm{mmol}), \mathrm{BuOK}(3 \mathrm{mg}, 0.02 \mathrm{mmol})$, triethylsilane ( $64 \mu \mathrm{L}, 0.40 \mathrm{mmol})$, 2-octyne (26 $\mathrm{mg}, 0.24 \mathrm{mmol})$ and cyclohexanecarboxaldehyde $(24 \mu \mathrm{L}, 0.20 \mathrm{mmol})$ were employed to give a 3 : 1 mixture of the regioisomers (S,E)-(1-cyclohexyl-2ethylideneheptyloxy)triethylsilane and $\quad(S, E)$-(1-cyclohexyl-2-methyloct-2enyloxy)triethylsilane (54 $\mathrm{mg}, \quad 0.16 \mathrm{mmol}, 79 \%$ yield, $75: 25$ regioselectivity), after column chromatography ( $\mathrm{SiO}_{2}$, hexanes) as a colorless oil. ${ }^{1} \mathrm{H} \mathrm{NMR}\left(400 \mathrm{MHz}, \mathrm{CDCl}_{3}\right)$ $\delta 5.25(\mathrm{q}, J=6.8 \mathrm{~Hz}, 1 \mathrm{H}), 3.56(\mathrm{~d}, J=7.6 \mathrm{~Hz}, 1 \mathrm{H}), 1.86-1.98(\mathrm{~m}, 3 \mathrm{H}), 1.60-1.74(\mathrm{~m}$, $3 \mathrm{H}), 1.56(\mathrm{~d}, J=6.8 \mathrm{~Hz}, 3 \mathrm{H}), 1.00-1.46(\mathrm{~m}, 11 \mathrm{H}), 0.74-0.93(\mathrm{~m}, 14 \mathrm{H}), 0.52(\mathrm{q}, J=8.0$ $\mathrm{Hz}, 6 \mathrm{H}) ;{ }^{13} \mathrm{C}\left(125 \mathrm{MHz}, \mathrm{CDCl}_{3}\right) \delta 141.5,121.4,83.1,41.7,32.7,30.2,29.0,26.8,26.6$, 26.2, 22.5, 14.0, 12.9, 6.9, 4.9 ; IR (Thin film, KBr, $\mathrm{cm}^{-1}$ ) 2953, 2929, 2875, 1457, 1067, 1006, 724; HRMS (EI) $\mathrm{m} / \mathrm{z}$ calculated for $\mathrm{M}^{+} 338.3005$, found 338.3015. Following the general procedure, Mosher ester was synthesized (with $R$-MTPA-Cl) followed by ${ }^{19} \mathrm{~F}$ NMR analysis which illustrated $76 \%$ ee (see S50 for characteristic diagnostic peaks at 70.98 and $-71.44 \mathrm{ppm}$ for enantiopure and racemic spectra of ${ }^{1} \mathrm{H}$ NMR). The Mosher ester of the minor isomer was also synthesized (with $R$-MTPA-Cl) followed by ${ }^{19} \mathrm{~F}$ NMR analysis which illustrated $76 \%$ ee.

(S,E)-(1-Cyclohexyl-2-methyloct-2-enyloxy)triethylsilane (entry 12)

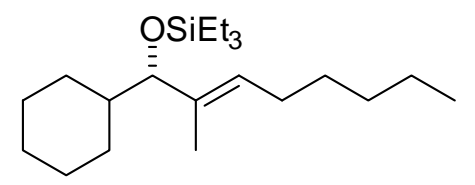


Following the general procedure, $\mathrm{Ni}(\mathrm{COD})_{2}(6 \mathrm{mg}, 0.02 \mathrm{mmol})$, ligand $(R, R) \mathbf{1 d}(12 \mathrm{mg}$, $0.02 \mathrm{mmol}$ ), $t \mathrm{BuOK}(5 \mathrm{mg}, 0.04 \mathrm{mmol})$, triethylsilane ( $64 \mu \mathrm{L}, 0.40 \mathrm{mmol}), 2$-octyne (26 $\mathrm{mg}, 0.24 \mathrm{mmol})$ and cyclohexanecarboxaldehyde $(24 \mu \mathrm{L}, 0.2 \mathrm{mmol})$ were employed to give $6: 1$ mixture of the regioisomers (S,E)-(1-cyclohexyl-2-methyloct-2enyloxy)triethylsilane and (S,E)-(1-cyclohexyl-2-ethylideneheptyloxy)triethylsilane (32 mg, 0.095 mmol, $47 \%$ yield, 6:1 regioselectivity), after column chromatography ( $\mathrm{SiO}_{2}$, hexanes) as a colorless oil. ${ }^{1} \mathrm{H}$ NMR $\left(400 \mathrm{MHz}, \mathrm{CDCl}_{3}\right) \delta 5.17(\mathrm{td}, J=7.2,0.8 \mathrm{~Hz}, 1 \mathrm{H})$, $3.49(\mathrm{~d}, J=8.4 \mathrm{~Hz}, 1 \mathrm{H}), 1.88-2.04(\mathrm{~m}, 3 \mathrm{H}), 1.54-1.74(\mathrm{~m}, 3 \mathrm{H}), 1.48(\mathrm{~s}, 3 \mathrm{H}), 1.00-1.38$ (m, 11H), 0.64-0.92 (m, 14H), $0.52(\mathrm{q}, J=8.0 \mathrm{~Hz}, 6 \mathrm{H}) ;{ }^{13} \mathrm{C}\left(125 \mathrm{MHz}, \mathrm{CDCl}_{3}\right) \delta$ 136.2, $127.2,84.1,41.2,31.5,29.9,29.5,29.1,27.3,26.6,26.2,26.1,22.5,14.0,10.9,6.8,4.8$. Following the general procedure, Mosher ester was synthesized (with $R$-Mosher acid) followed by ${ }^{19} \mathrm{~F}$ NMR analysis which illustrated $79 \%$ ee (see S52 for characteristic diagnostic peaks at -70.8 and $-71.5 \mathrm{ppm}$ for enantiopure and racemic spectra of ${ }^{19} \mathrm{~F}$ ).

(S,E)-(2-Methylcyclotetradec-2-enyloxy)triethylsilane (4a)

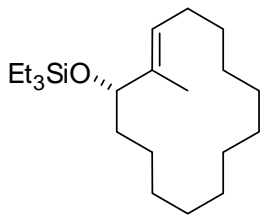

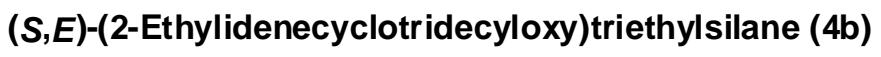

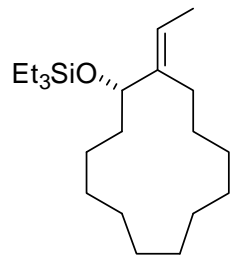

$\mathrm{Ni}(\mathrm{COD})_{2}(5 \mathrm{mg}, 0.017 \mathrm{mmol})$, ligand $(R, R) \mathbf{1 f}(14 \mathrm{mg}, 0.017 \mathrm{mmol}), t \mathrm{BuOK}(2 \mathrm{mg}$, $0.017 \mathrm{mmol}$ ) were weighed in an oxygen free inert atmosphere glovebox in a flame-dried $50 \mathrm{~mL}$ round-bottomed flask with a stir bar and sealed with a septum. $5 \mathrm{~mL}$ toluene is added to the mixture under nitrogen atmosphere at $\mathrm{rt}$ and stirred for $10 \mathrm{~min}$, followed by 
dilution with $20 \mathrm{~mL}$ toluene. Triethylsilane ( $60 \mu \mathrm{L}, 0.340 \mathrm{mmol})$ was added, followed by syringe drive addition of pentadec-13-enal $(50 \mathrm{mg}, 0.167 \mathrm{mmol})$ in $10 \mathrm{~mL}$ toluene over $2 \mathrm{~h}$. The reaction was quenched by stirring the mixture in air for $30 \mathrm{~min}$. and concentrated by rotary evaporation. The crude mixture was purified by column chromatography $\left(\mathrm{SiO}_{2}\right.$, 1:20 ethyl acetate/hexanes) to afford an 86:14 mixture of the regioisomers $(S, E)-(2-$ methylcyclotetradec-2-enyloxy)triethylsilane (4a) and $(S, E)-(2-$ ethylidenecyclotridecyloxy)triethylsilane (4b) (42 mg, $0.124 \mathrm{mmol}, 76 \%$ yield) after column chromatography ( $\mathrm{SiO}_{2}, 1: 20$ ethyl acetate/hexanes) as a colorless oil. ${ }^{1} \mathrm{H}$ NMR $\left(400 \mathrm{MHz}, \mathrm{CDCl}_{3}\right) \delta 5.40$ (q, $J=6.8 \mathrm{~Hz}, 0.15 \mathrm{H}$, minor isomer), 5.16-5.24 (dd, J=9.6, 5.2 $\mathrm{Hz}, 0.85 \mathrm{H}$, major isomer), 3.95 (dd, $J=10.4,4.0 \mathrm{~Hz}, 0.85 \mathrm{H}$, major isomer), 3.90 (t, $J=$ $5.6 \mathrm{~Hz}, 0.15 \mathrm{H}$, minor isomer), 2.05-2.20 (m, 1H), 1.82-2.00 (m, 1H), 1.57 (d, $J=6.8 \mathrm{~Hz}$, $0.45 \mathrm{H}$, minor isomer), $0.51(\mathrm{~s}, 2.55 \mathrm{H}$, major isomer), $0.98-1.48(\mathrm{~m}, 20 \mathrm{H}), 0.88(\mathrm{t}, J=8.0$ $\mathrm{Hz}, 9 \mathrm{H}), 0.52(\mathrm{q}, J=8.0 \mathrm{~Hz}, 6 \mathrm{H}) ;{ }^{13} \mathrm{C}\left(125 \mathrm{MHz}, \mathrm{CDCl}_{3}\right.$ ); (major and minor) $\delta 142.3$, $137.1,127.1,120.0,78.5,77.5,35.5,34.8,27.9,27.6,26.9,26.8,26.7,26.5,26.4,26.0$, $25.9,25.7,25.3,25.0,24.4,24.3,24.2,23.5,23.4,23.1,22.7,13.2,10.5,6.9,6.8,4.8$, 4.7; IR (Thin film, KBr, $\mathrm{cm}^{-1}$ ) 2931, 1457, 1062, 1006, 742; HRMS (EI) m/z calculated for $\mathrm{M}^{+}$338.3005, found 338.2994. Following the general procedure, silyl deprotection gave the alcohols which were separated by column chromatography (6:1/ethyl acetate : hexanes). Following the general procedure, Mosher ester of the major regioisomer was synthesized (with $R$-Mosher acid) followed by ${ }^{19} \mathrm{~F}$ NMR analysis which illustrated $79 \%$ ee (see S54 for characteristic diagnostic peaks between -71.5 to $-71.9 \mathrm{ppm}$ for enantiopure and racemic spectra of ${ }^{19} \mathrm{~F}$ NMR). Mosher ester of the minor isomer was also synthesized (with $R$-Mosher acid) followed by ${ }^{1} \mathrm{H}$ NMR analysis which illustrated $42 \%$ 
ee (see S55 for characteristic diagnostic peaks between 5.80 to $5.20 \mathrm{ppm}$ for enantiopure and racemic spectra of $\left.{ }^{1} \mathrm{H} \mathrm{NMR}\right)$

\section{Determination of absolute configuration of entry 10 via Mosher's ester analysis}
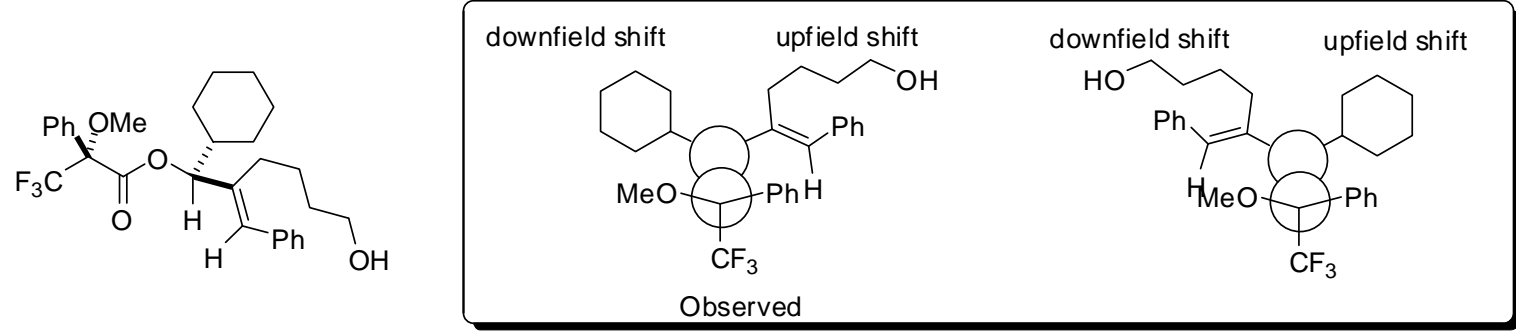

The absolute stereochemistry of entries $2,3,5,6,7,8,9,10,11,12$ and macrocycles $4 \mathbf{a}$ and 4b were analyzed based on Mosher's ester analysis using Mosher's acid or acid chloride ${ }^{4}$ The analysis was done as follows, using entry 10 as an example. Shielding of the alkenyl group in the major diastereomer was observed suggesting an absolute configuration of $(\mathrm{S})$ in the major diastereomer. Also, shielding of the $\mathrm{MeO}$ - signal in the minor diastereomer by the alkene supports this assignment, though this shielding effect is not observed in all cases due to different substitutions. The Mosher's ester of entry 3 had previously been analyzed and the absolute stereochemistry produced by this methodology was confirmed via conversion to a previously reported derivative. ${ }^{5}$

\section{References:}

1. Funk, T. W.; Berlin, J. M.; Grubbs R. H. J. Am. Chem. Soc. 2006, 128, 1840.

2. Black, K. D.; Gunstone F. D. Chem Phys Lipids 1996, 79, 79.

3. Mahandru, G.M.; Liu, G.; Montgomery, J. J. Am. Chem. Soc. 2004, 126, 3698.

4. (a) Mosher, H. S.; Dale, J. A. J. Am. Chem. Soc. 1973, 95, 512-219. (b) Dale, J. A.; Dull, D. L.; Mosher, H. S. J. Org. Chem. 1969, 34, 2543-2549.

5. Miller, K. M.; Haung, W.-S.; Jamison, T. F. J. Am. Chem. Soc. 2003, 125, 3442. 


\section{$1 R, 2 R-N^{1}, N^{2}$-bis(2-cyclohexylphenyl)-1,2-diphenylethane-1,2-diamine}

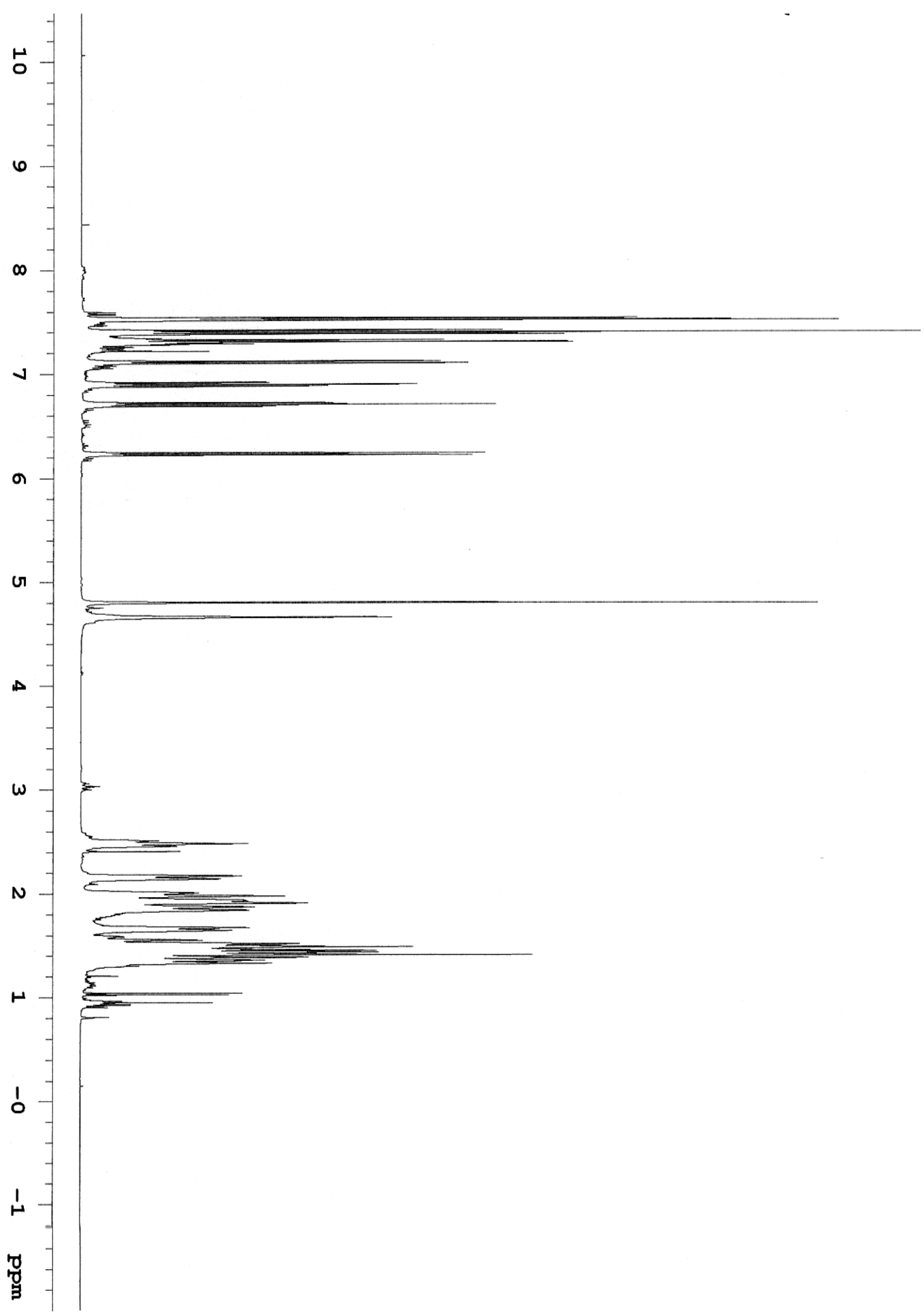




\section{R,5R-1,3-bis(2-cyclohexylphenyl)-4,5-dihydro-1H-imidazol-3-ium}

tetrafluoroborate (1d)

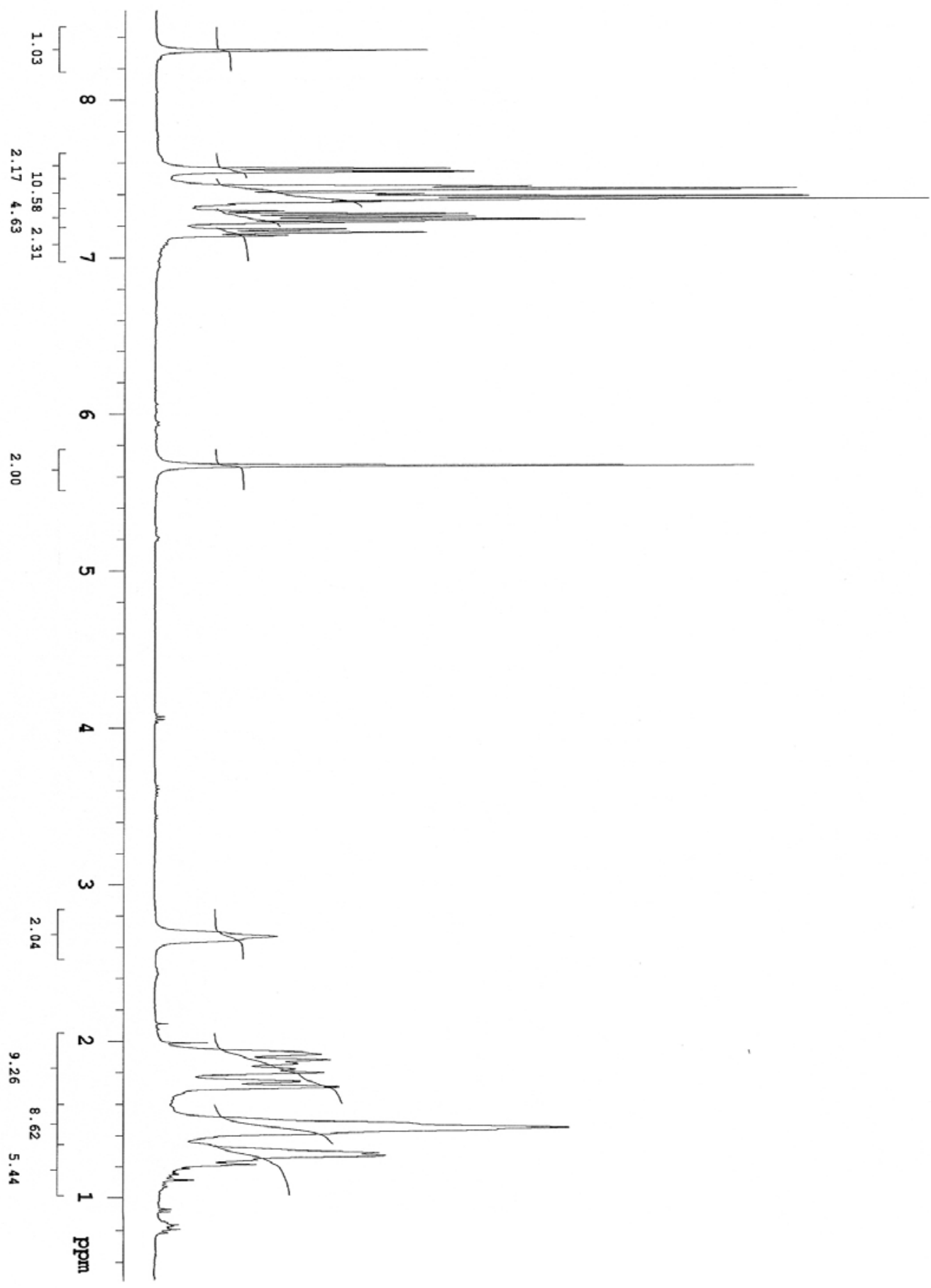


$1 R, 2 R-N^{1}, N^{2}$-bis(2,6-dimethylphenyl)-1,2-diphenylethane-1,2-diamine

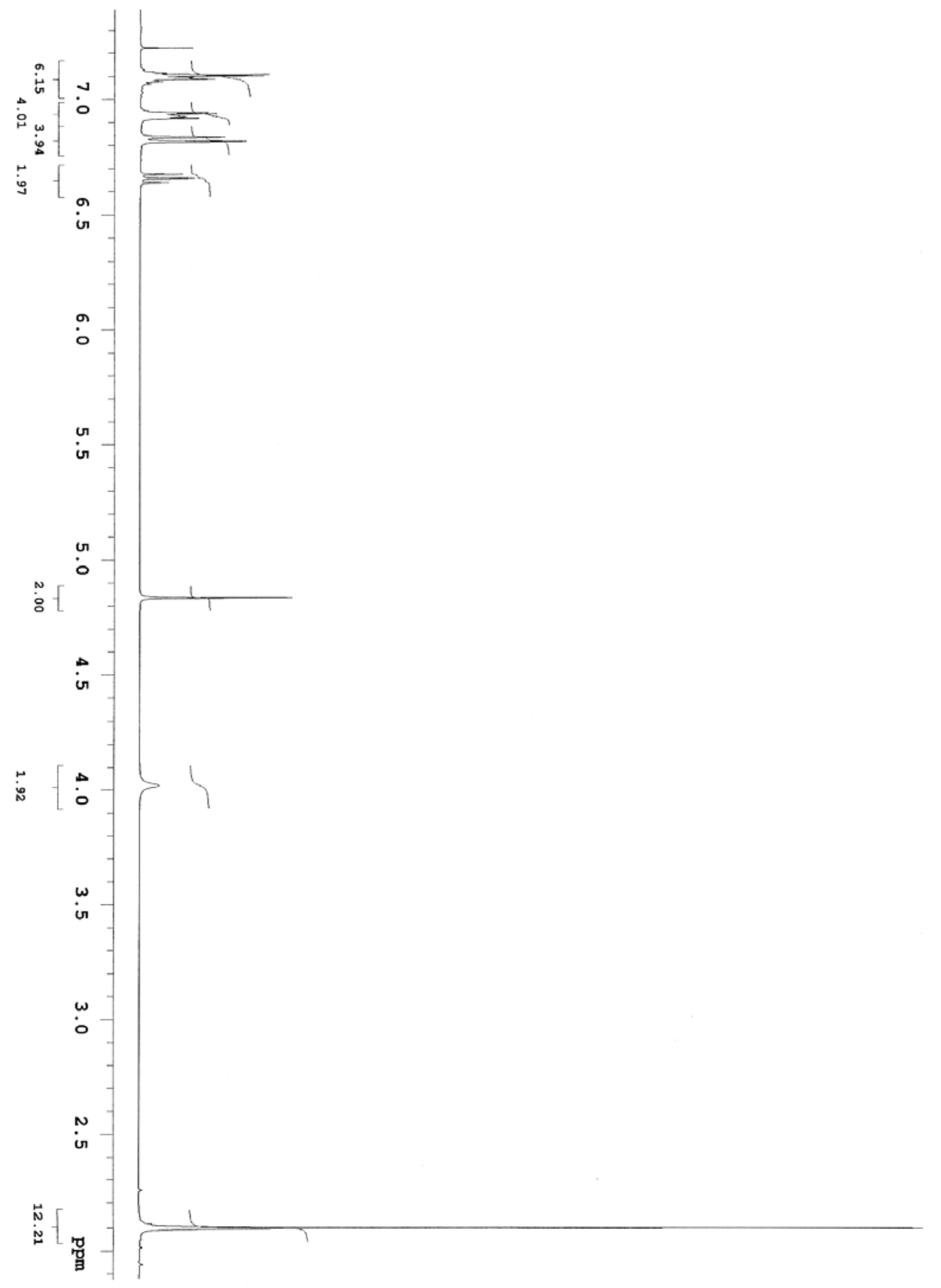


4R,5R-1,3-bis(2,6-dimethylphenyl)-4,5-diphenyl-4,5-dihydro-1H-imidazol-3-ium tetrafluoroborate (1e)

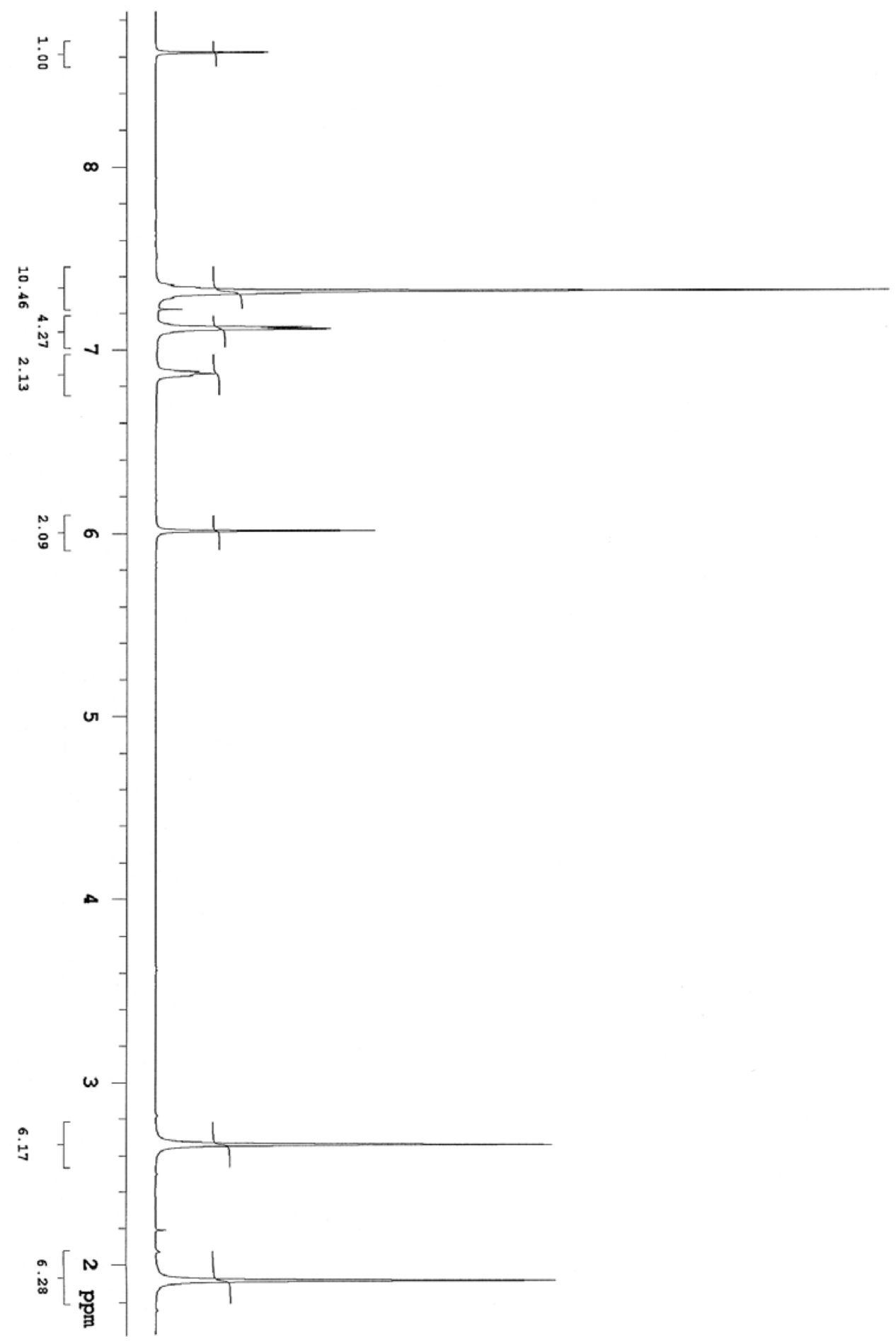




\section{2-Bromo-3,5-dicyclohexyl toluene}

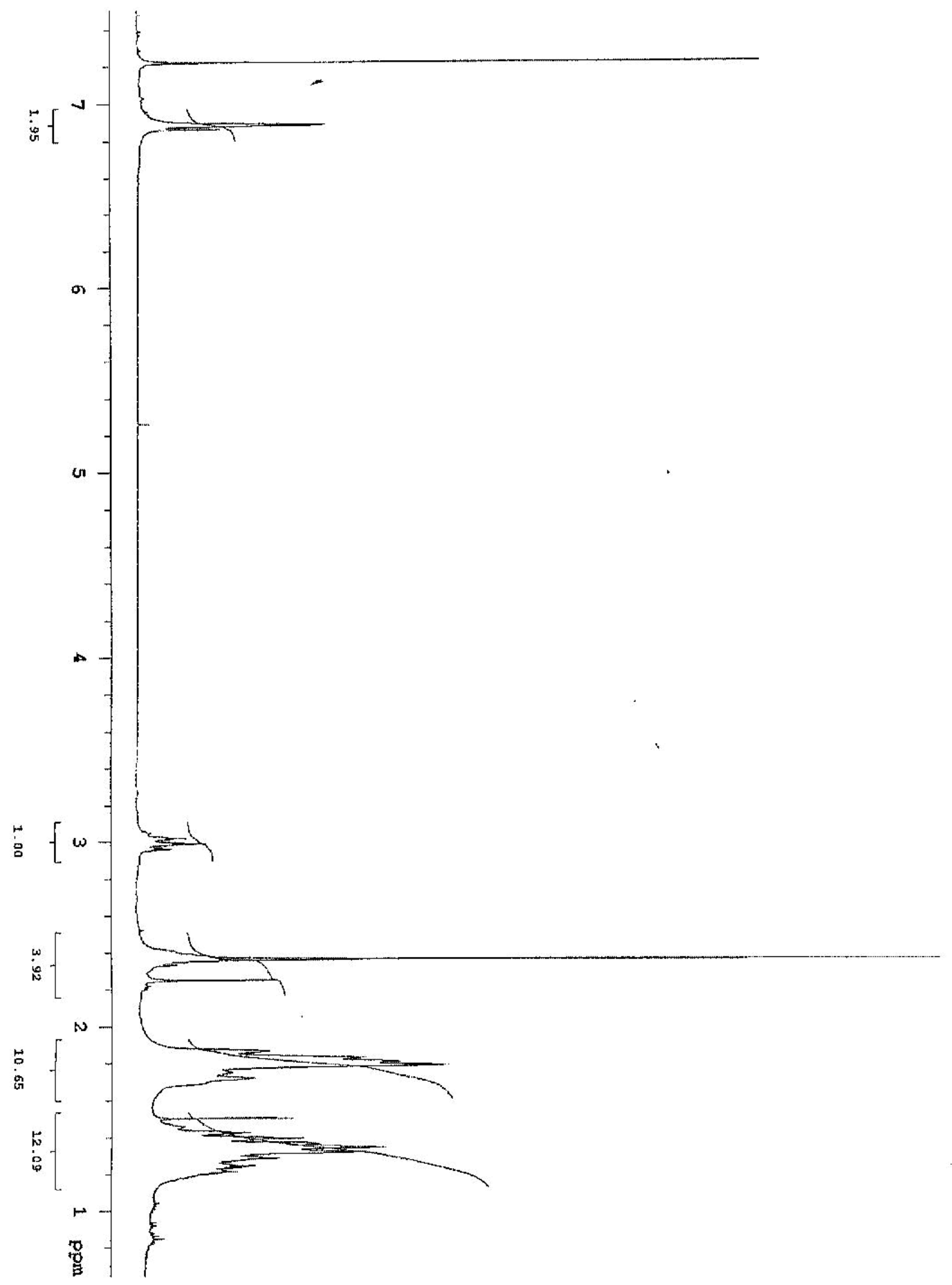


$1 R, 2 R-N^{1}, N^{2}$-bis(2,4-dicyclohexyl-6-methylphenyl)-1,2-diphenylethane-1,2-diamine

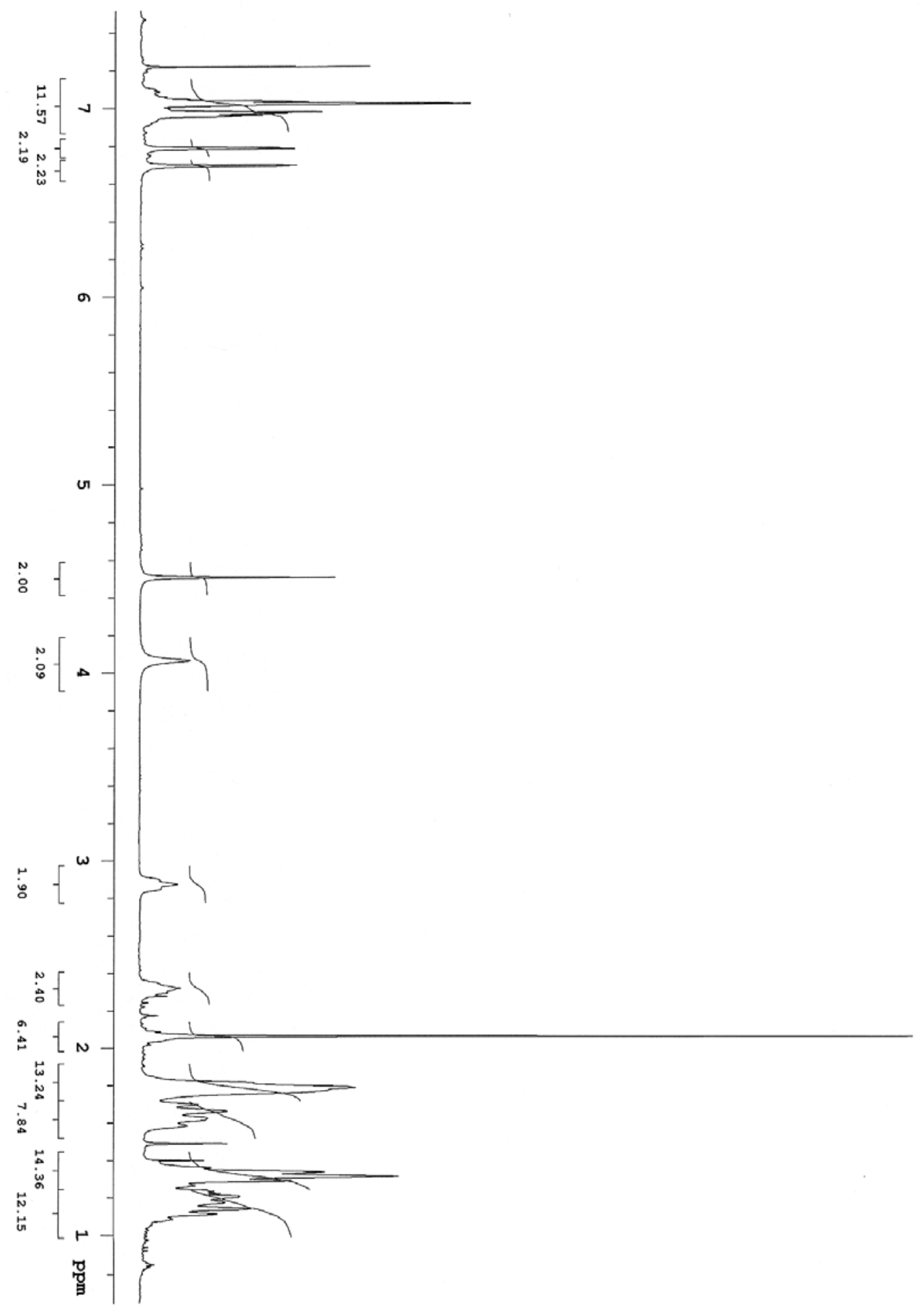


4R,5R-1,3-bis(2,4-dicyclohexyl-6-methylphenyl)-4,5-diphenyl-4,5-dihydro-1Himidazol-3-iumtetrafluoroborate (1f)

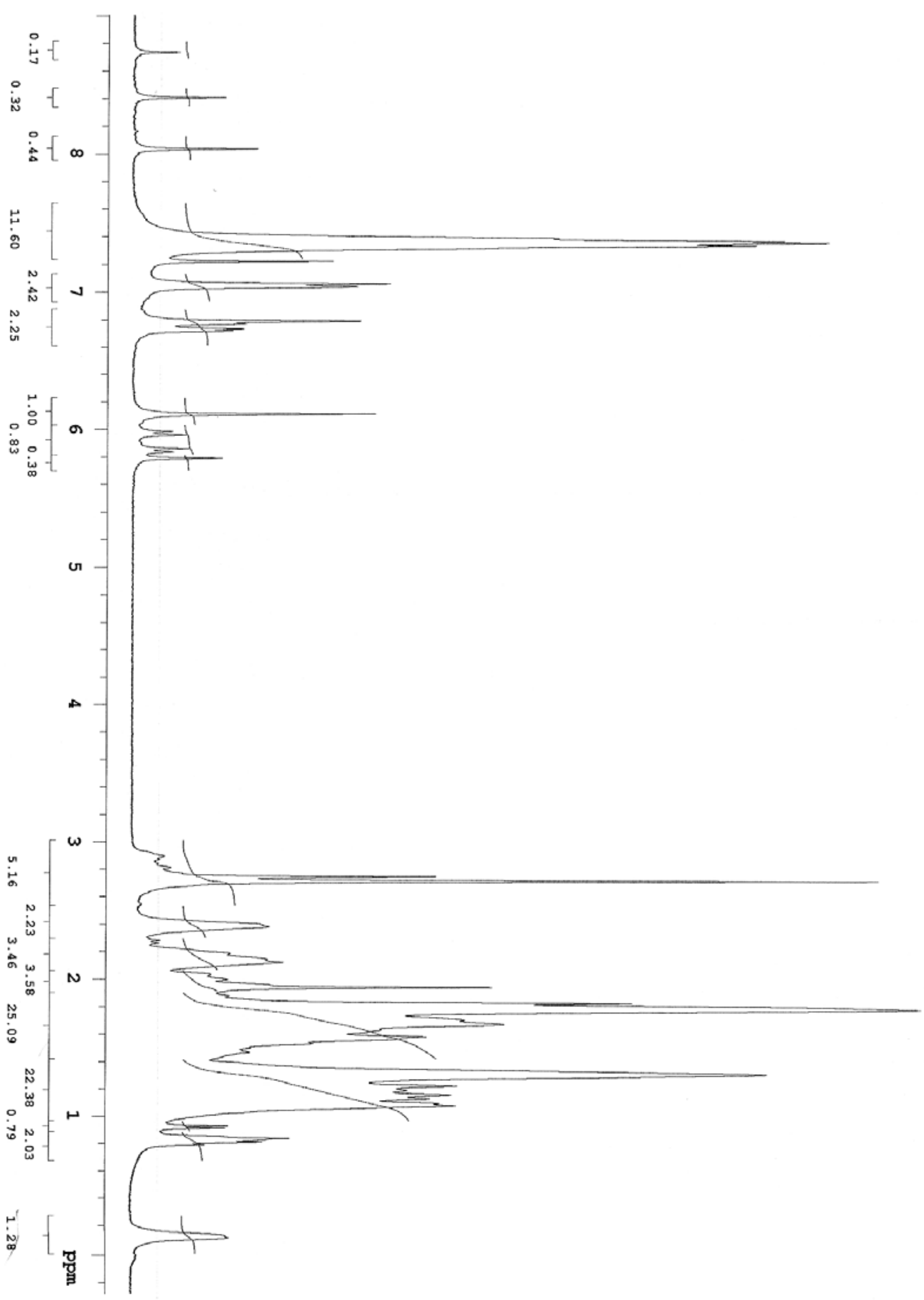




\section{R,E)-triethyl(2-methyl-1,3-diphenylallyloxy)silane (entry 1) (Chiral HPLC traces)}

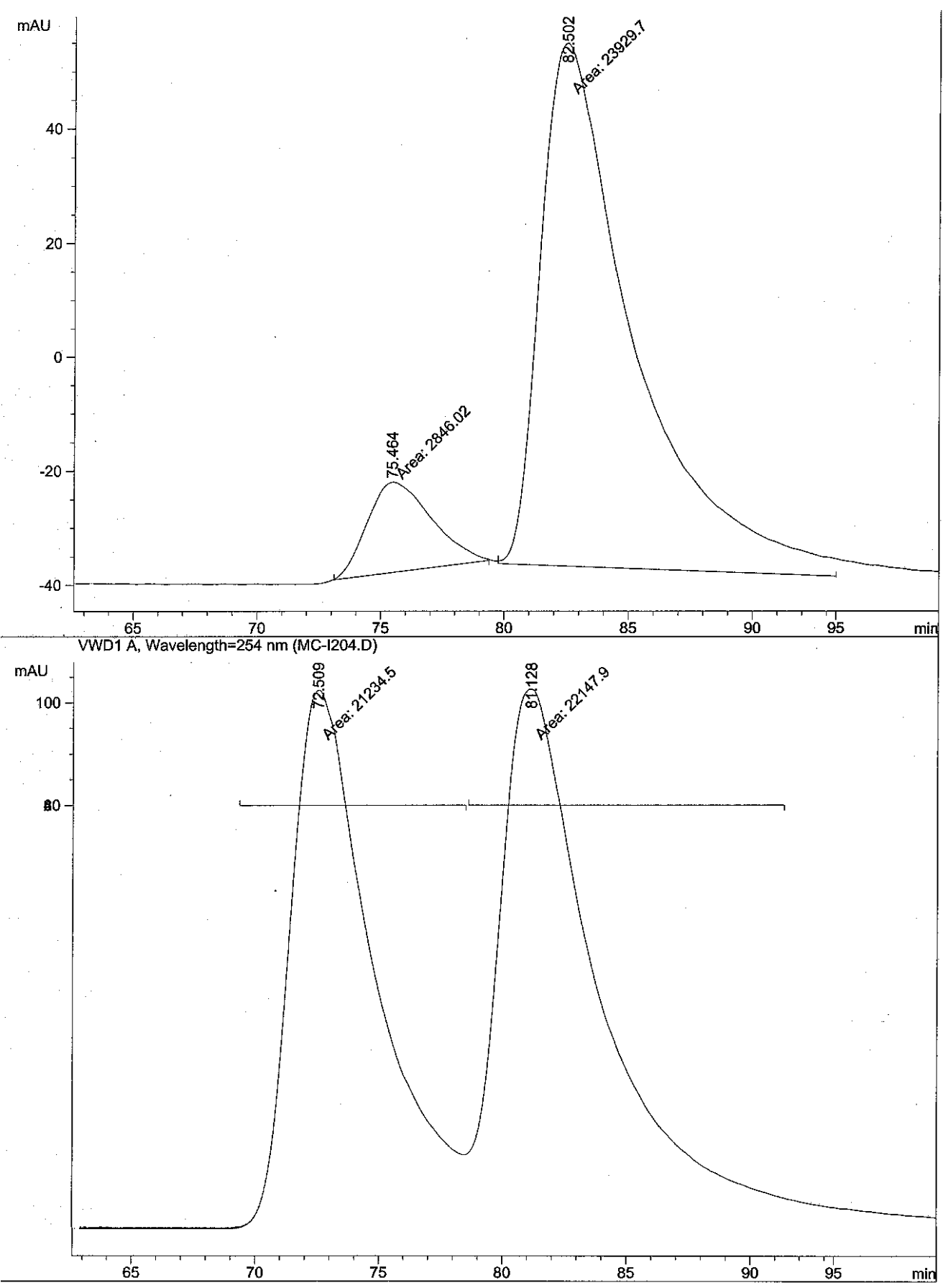


(R,E)-(2-ethyl-1-phenylpent-2-enyloxy)triethylsilane (entry 2)

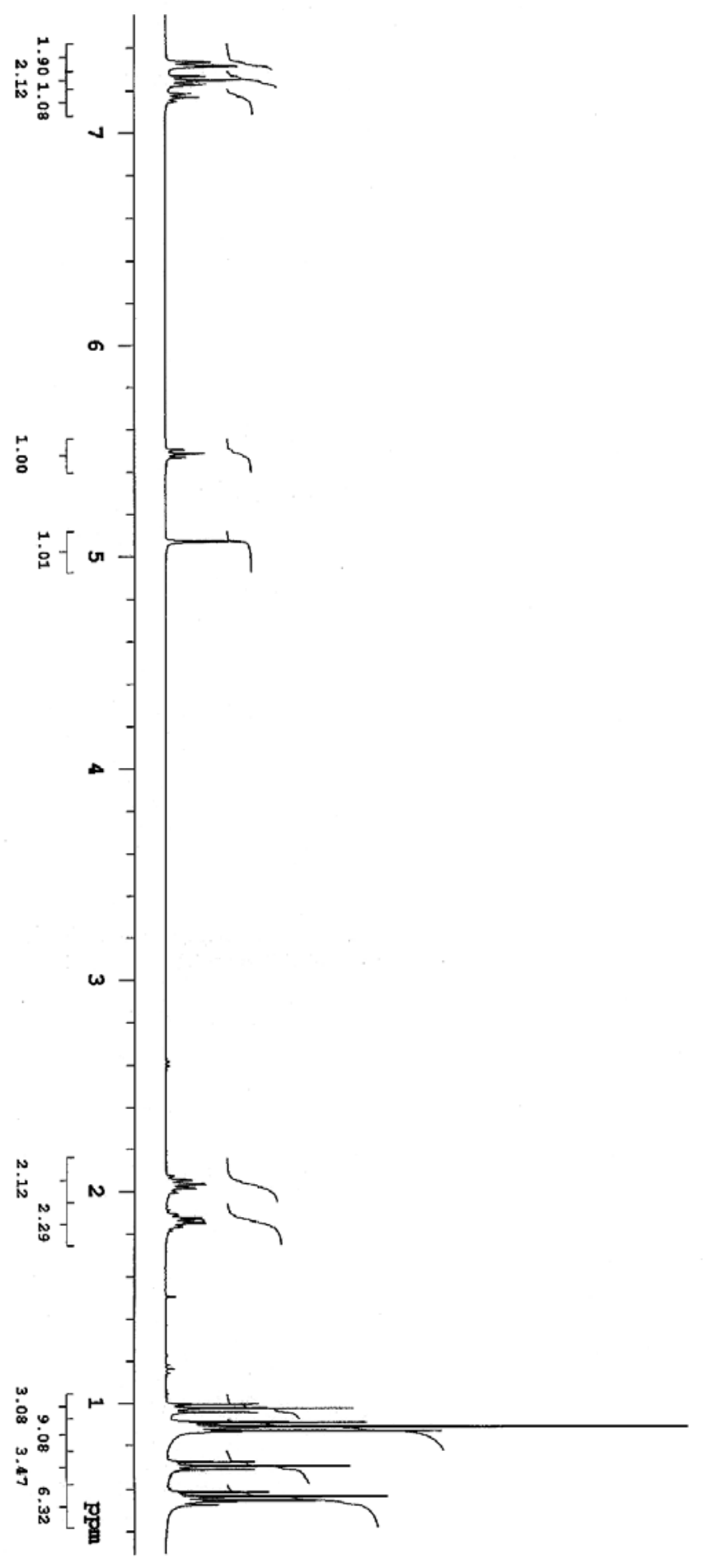




\section{(R,E)-(2-ethyl-1-phenylpent-2-enyloxy)triethylsilane (entry 2)}

${ }^{1} \mathrm{H}$ NMR spectra of $R$-Mosher ester
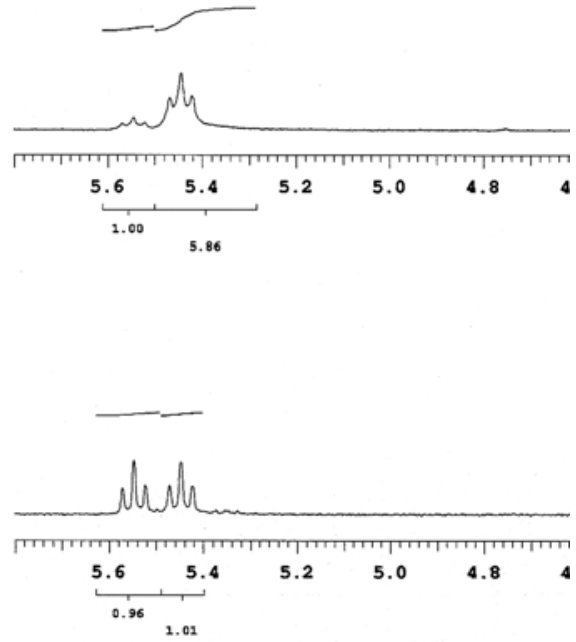

${ }^{19} \mathrm{~F}$ NMR spectra of $R$-Mosher ester

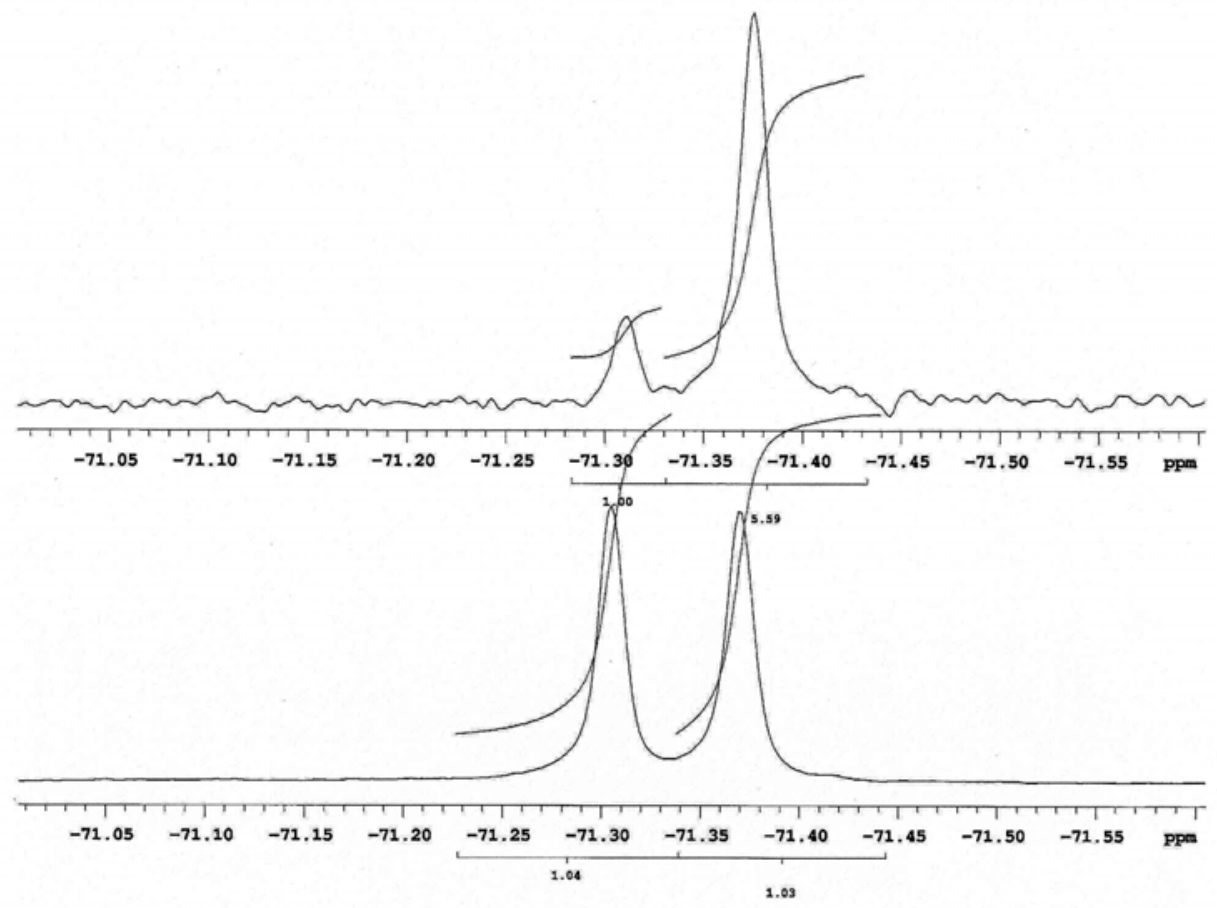


$(S, E)$-(2,4-Dimethyl-1-phenylpent-1-en-3-yloxy)triethylsilane (entry 3) ${ }^{1} \mathrm{H}$ NMR

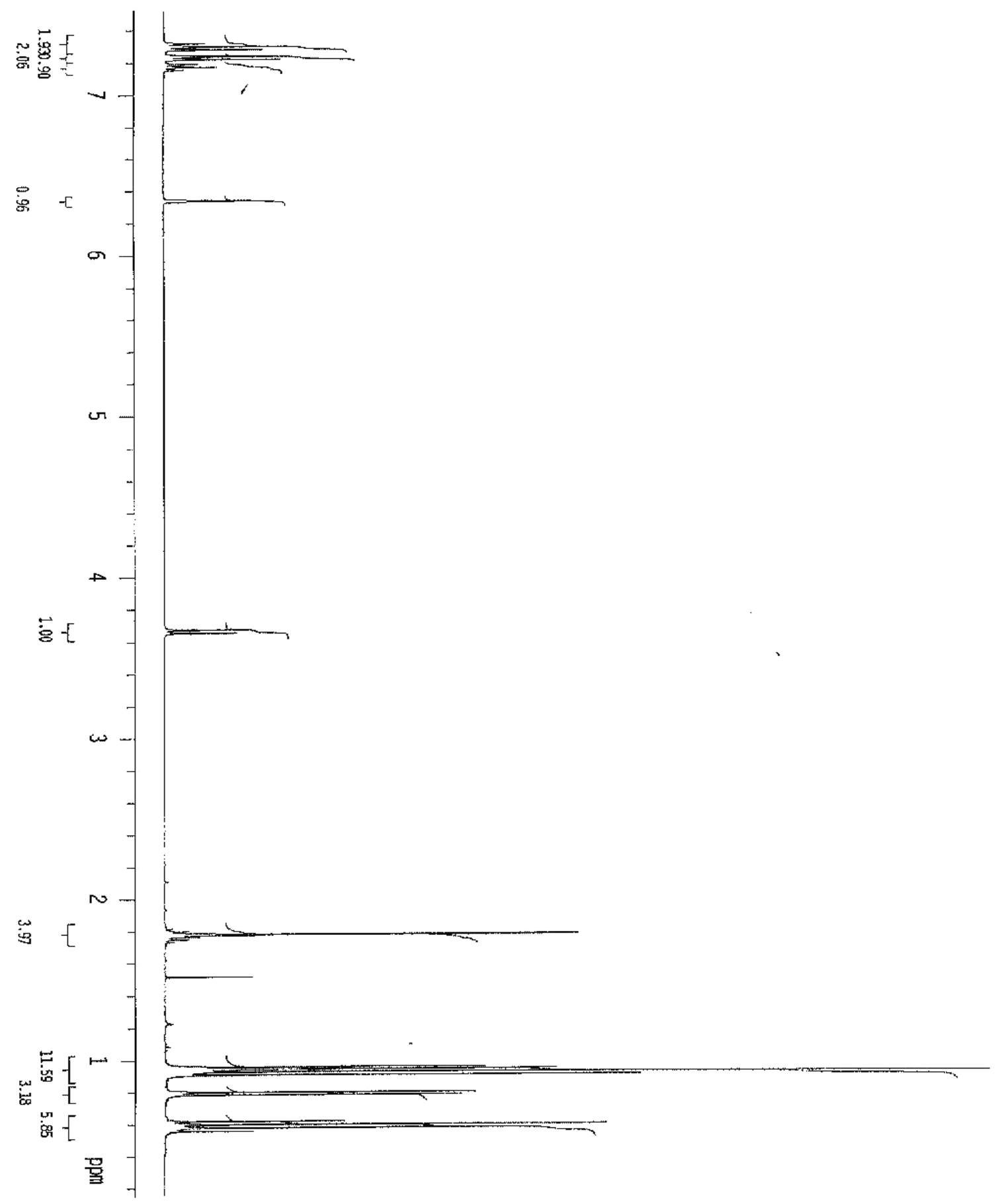


$(S, E)$-(2,4-Dimethyl-1-phenylpent-1-en-3-yloxy)triethylsilane (entry 3) (R)-Mosher's ester analysis ( ${ }^{1} \mathrm{NMR}$ and ${ }^{19} \mathrm{~F}$ NMR)
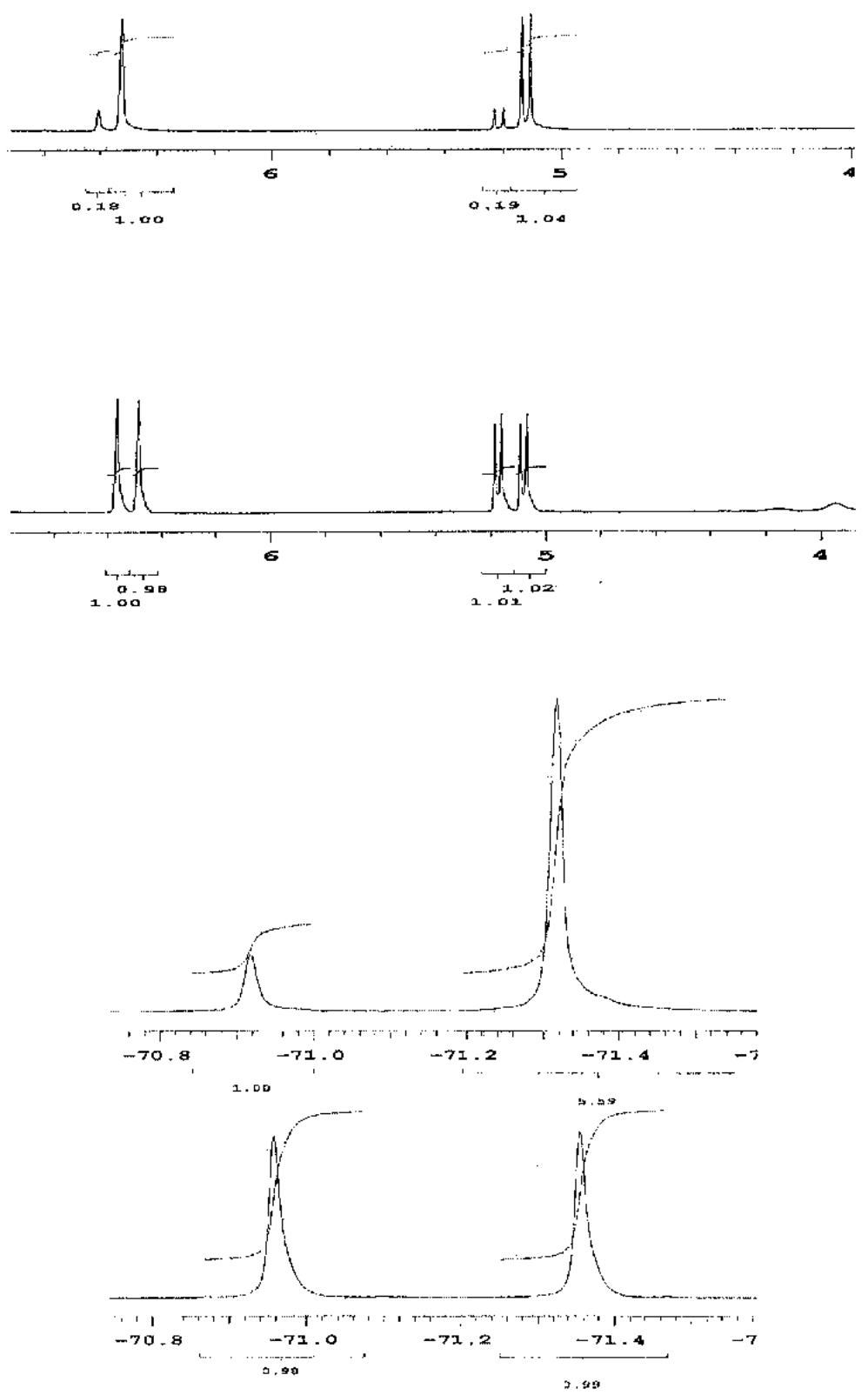
(S,E)-triethyl(4-ethylidene-2-methyl-7-phenylheptan-3-yloxy)silane (entry 4)

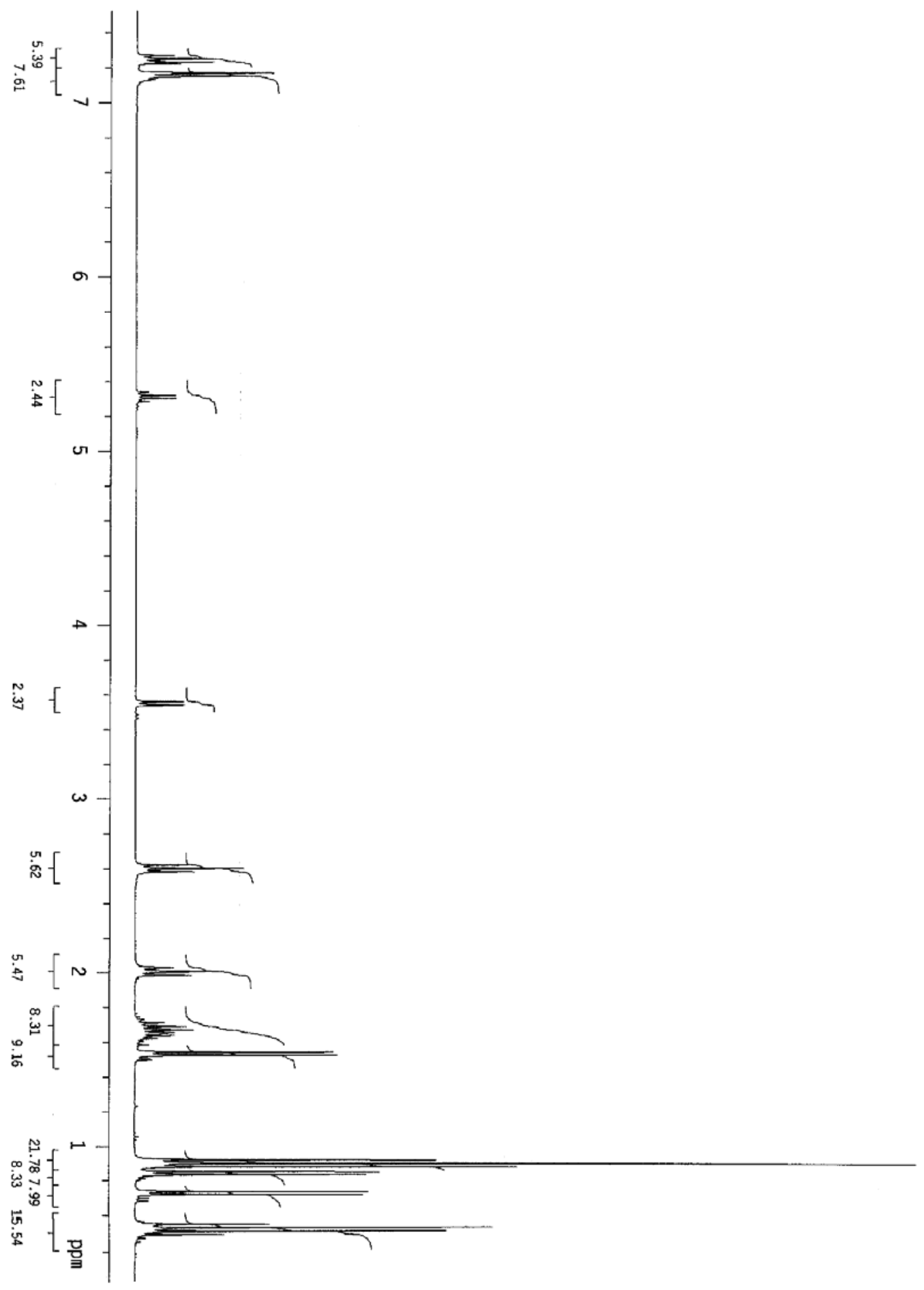




\section{(S,E)-triethyl(4-ethylidene-2-methyl-7-phenylheptan-3-yloxy)silane (entry 4) (Chiral}

\section{HPLC traces)}

Current Chromatogram (s)

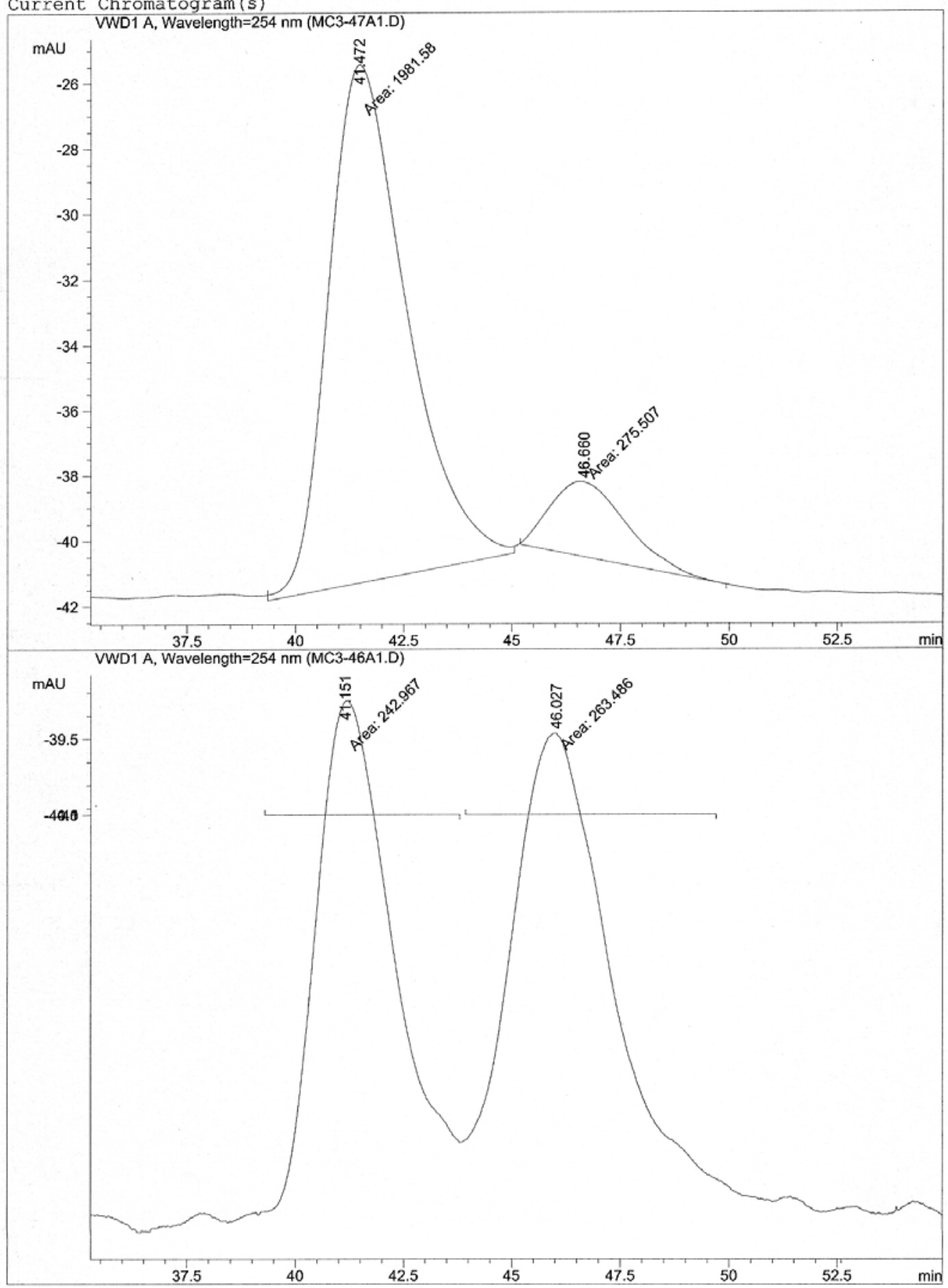


(R,E)-(1-cyclohexyl-2-ethylpent-2-enyloxy)triethylsilane (entry 5)

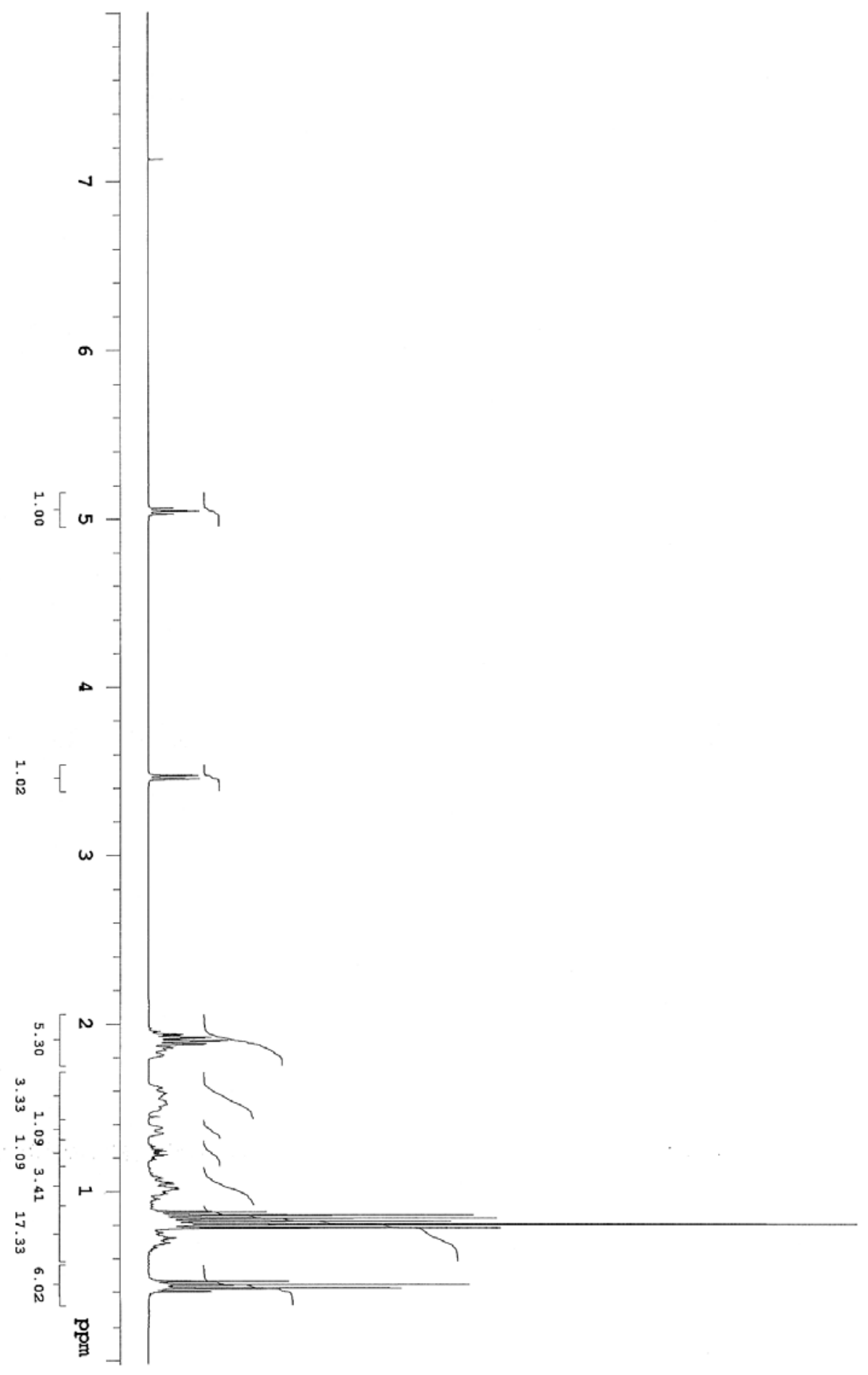




\section{(R,E)-(1-cyclohexyl-2-ethylpent-2-enyloxy)triethylsilane (entry 5)}

${ }^{1} \mathrm{H}-\mathrm{NMR}$ of $R$-Mosher ester

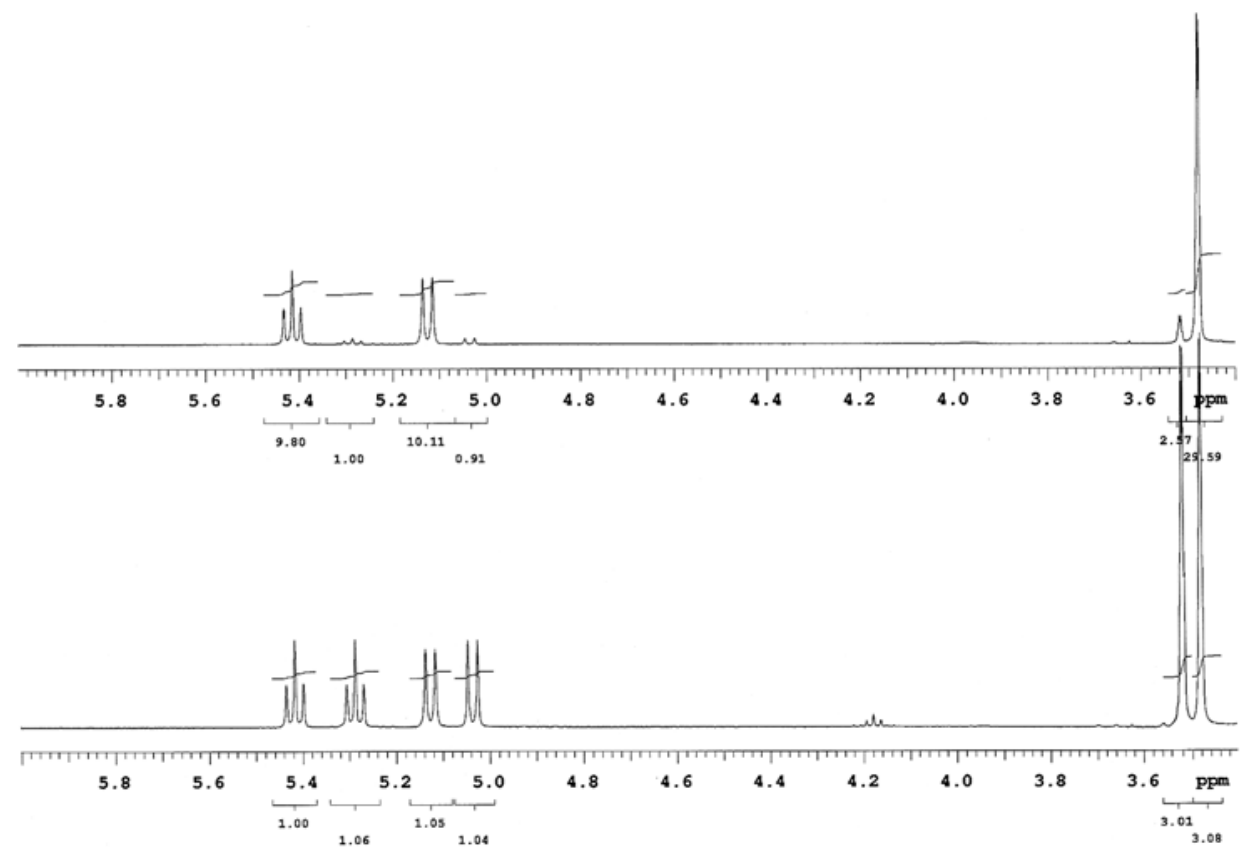

${ }^{19} \mathrm{~F}$ NMR of $R$-Mosher ester
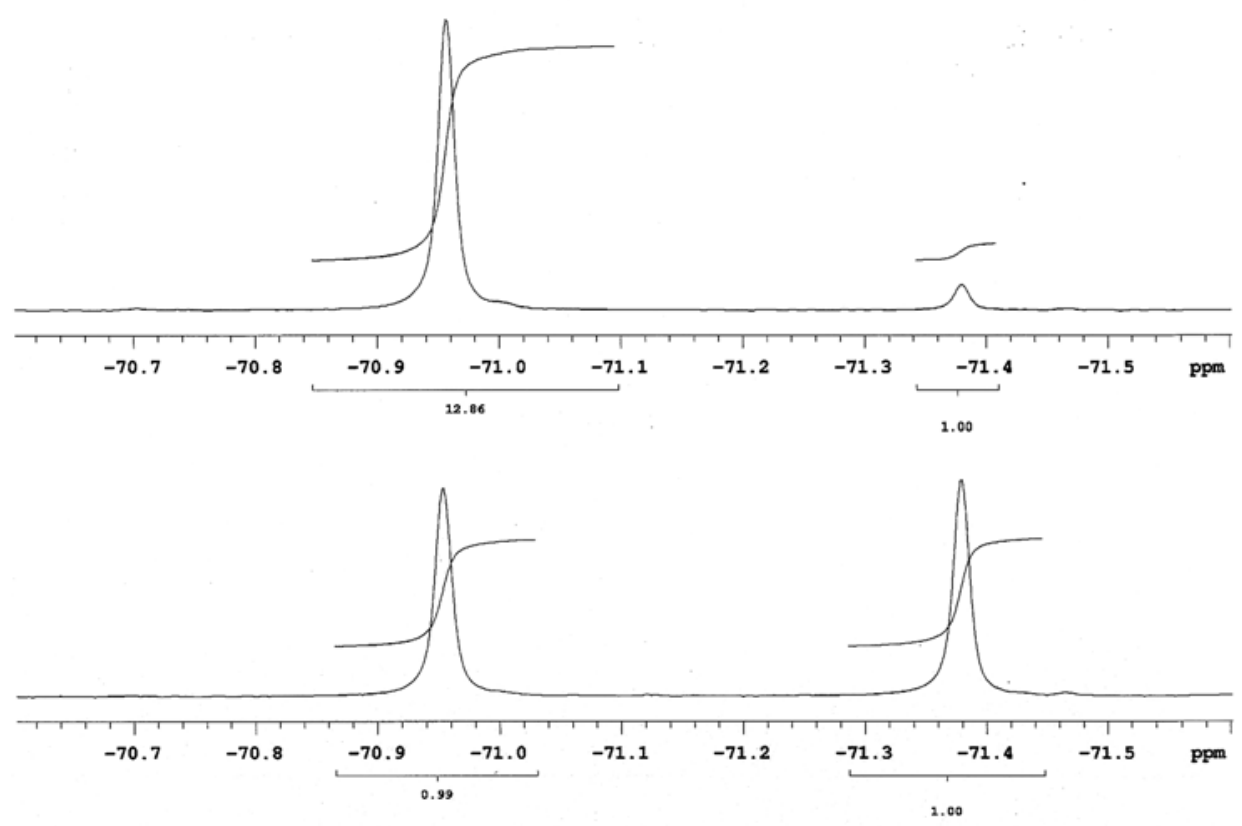
(S,E)-triethyl(4-ethyl-1-phenylhept-4-en-3-yloxy)silane (entry 6)

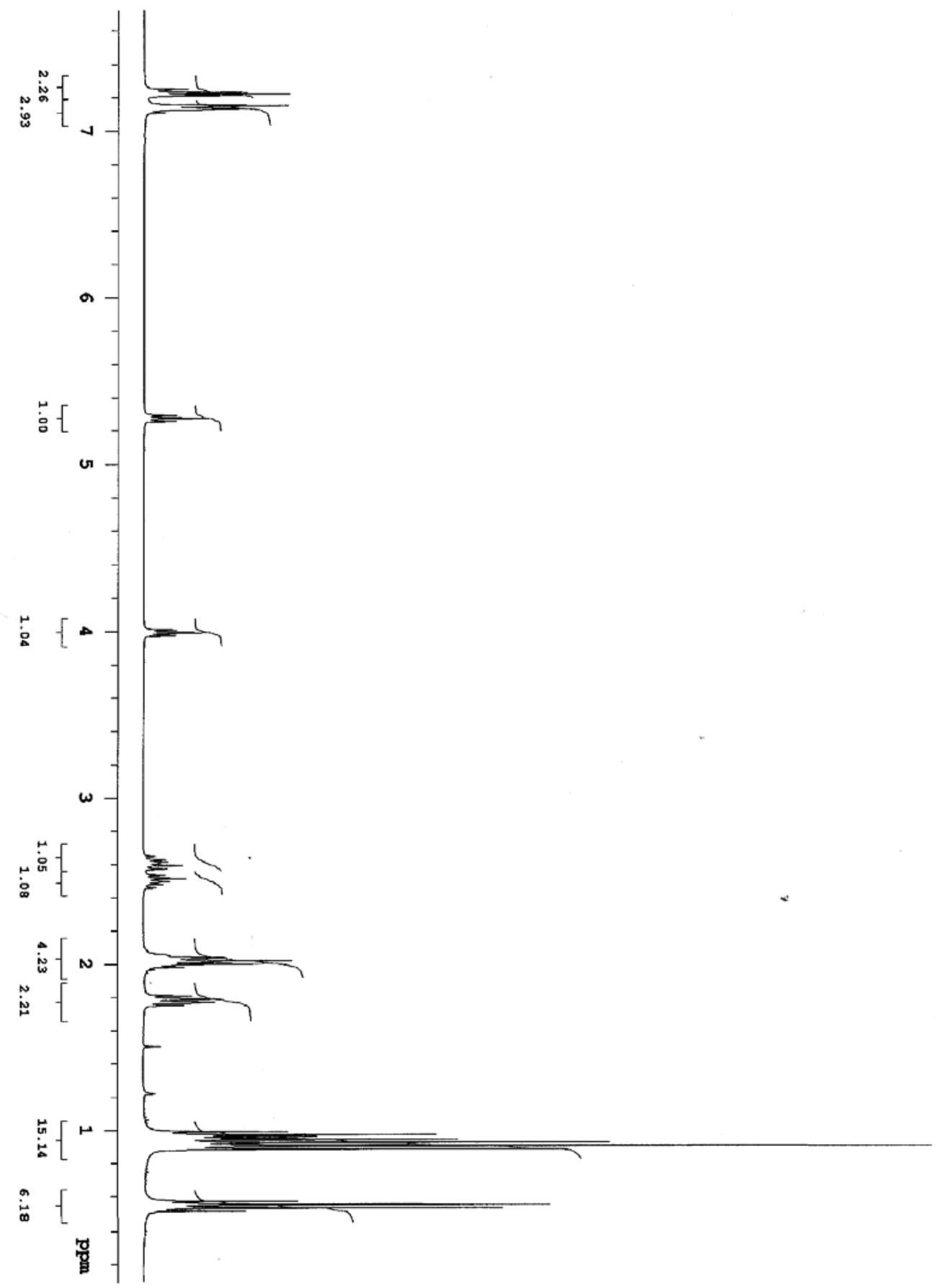




\section{(S,E)-triethyl(4-ethyl-1-phenylhept-4-en-3-yloxy)silane (entry 6)}

${ }^{1} \mathrm{H}-\mathrm{NMR}$ of $S$-Mosher ester
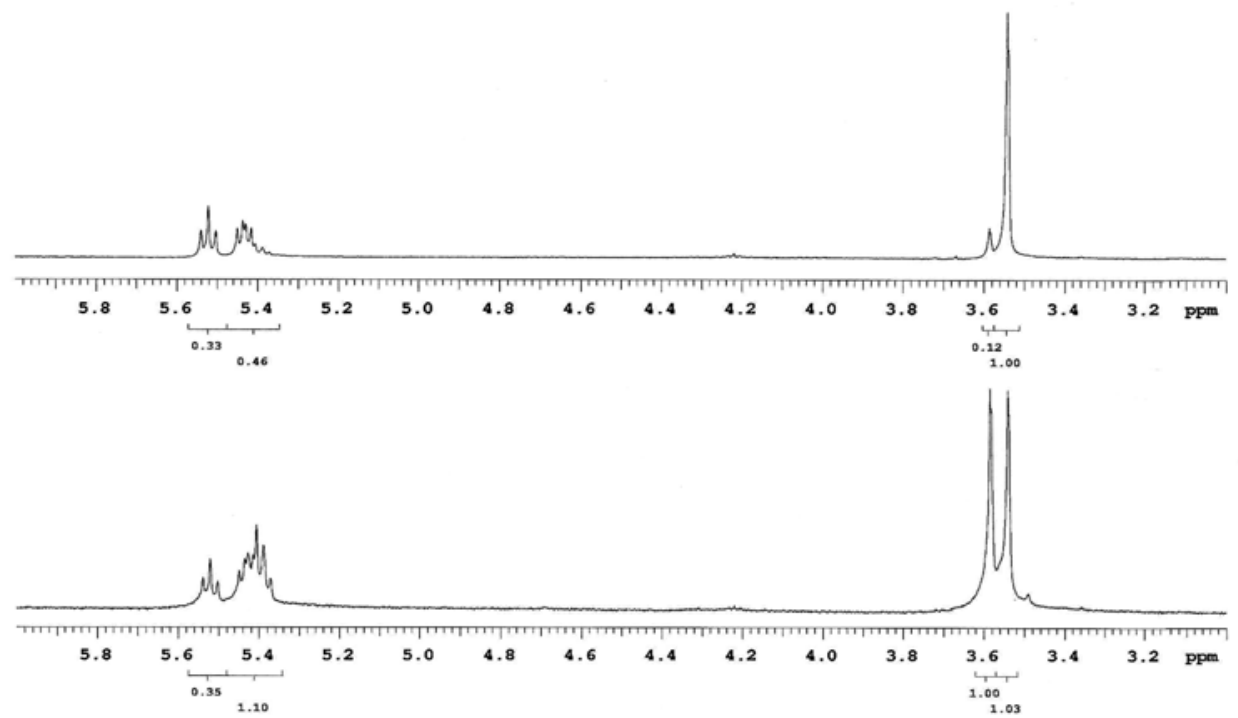

${ }^{19}$ F-NMR of S-Mosher ester

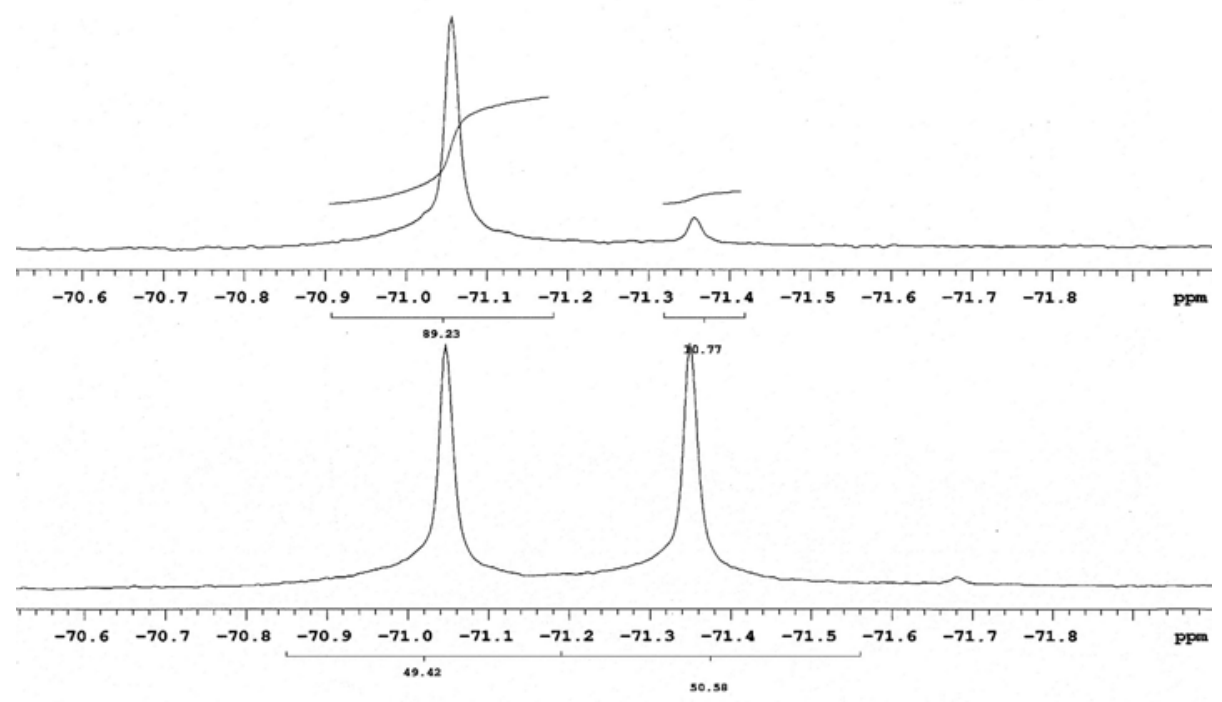




\section{(S,E)-(1-cyclohexyl-2-methyl-3-phenylallyloxy)triethylsilane (entry 7)}

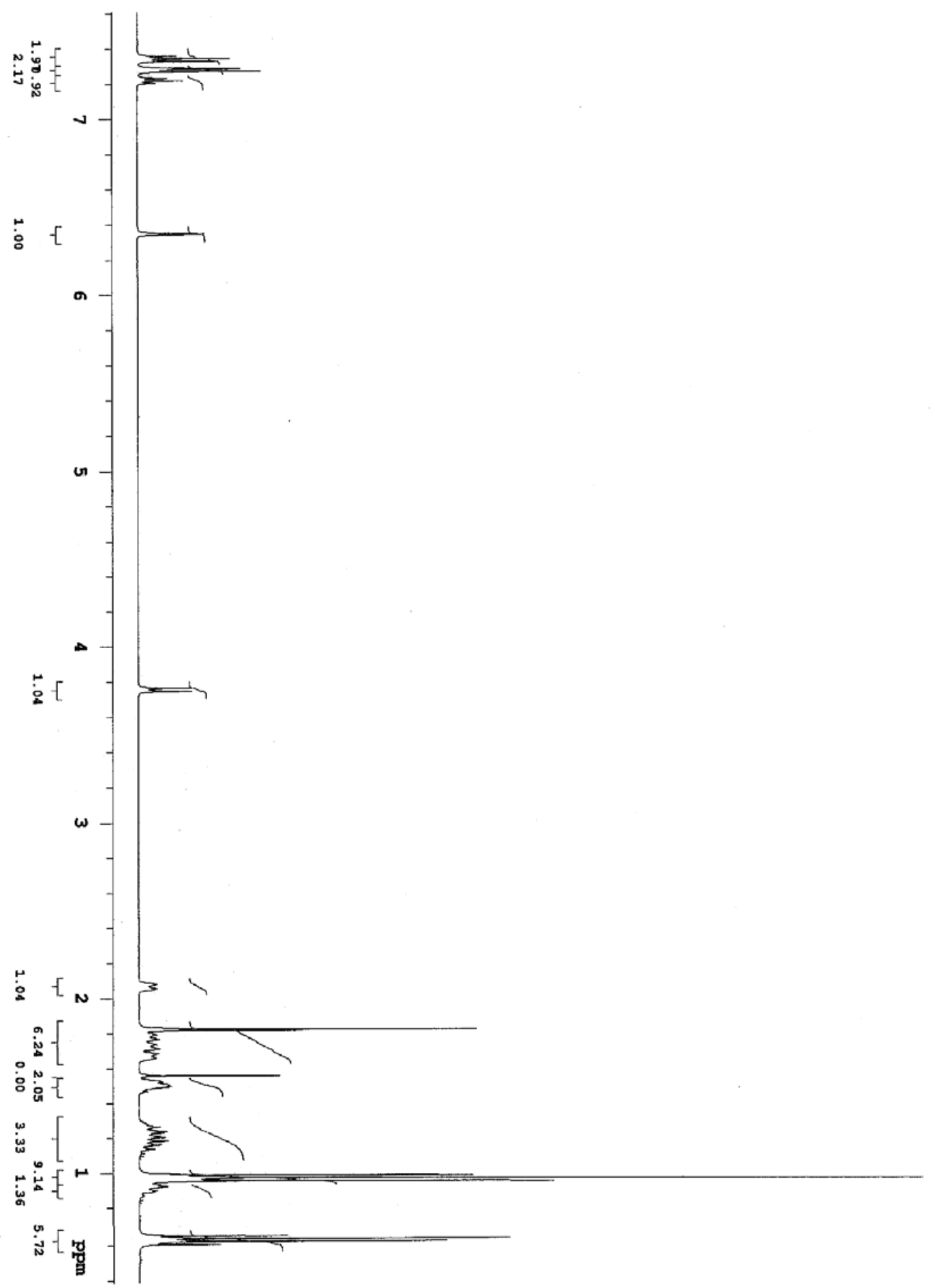




\section{(S,E)-(1-cyclohexyl-2-methyl-3-phenylallyloxy)triethylsilane (entry 7)}

${ }^{1} \mathrm{H}-\mathrm{NMR}$ of $R$-Mosher ester
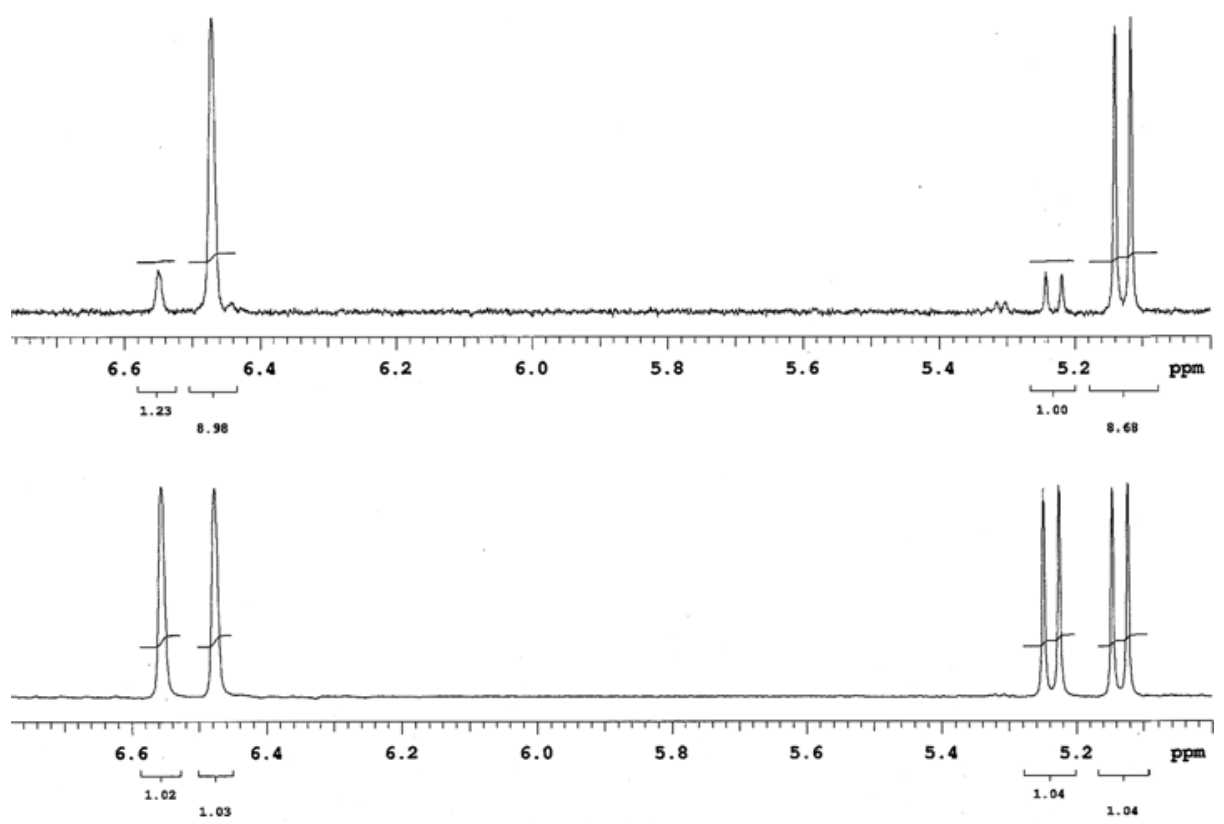

${ }^{19}$ F-NMR of $R$-Mosher ester

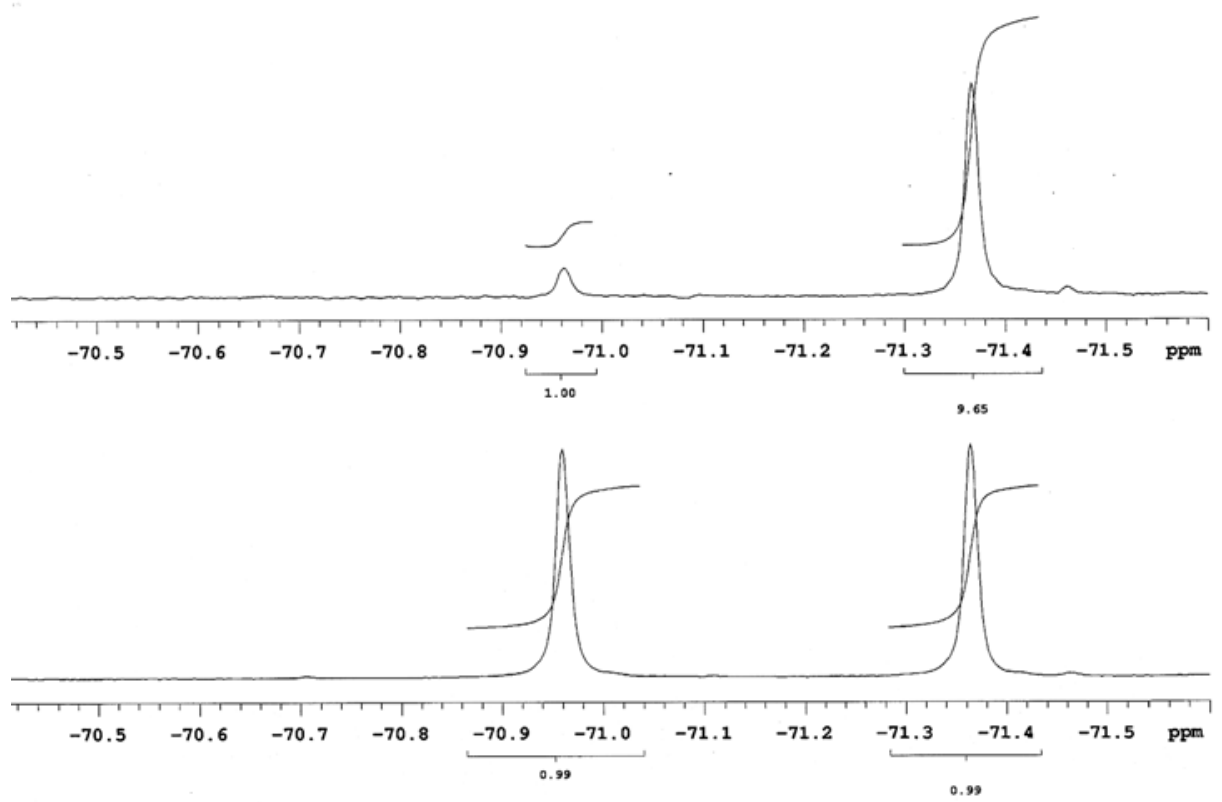


(S,E)-(1-cyclohexylnon-2-enyloxy)triethylsilane (entry 8)

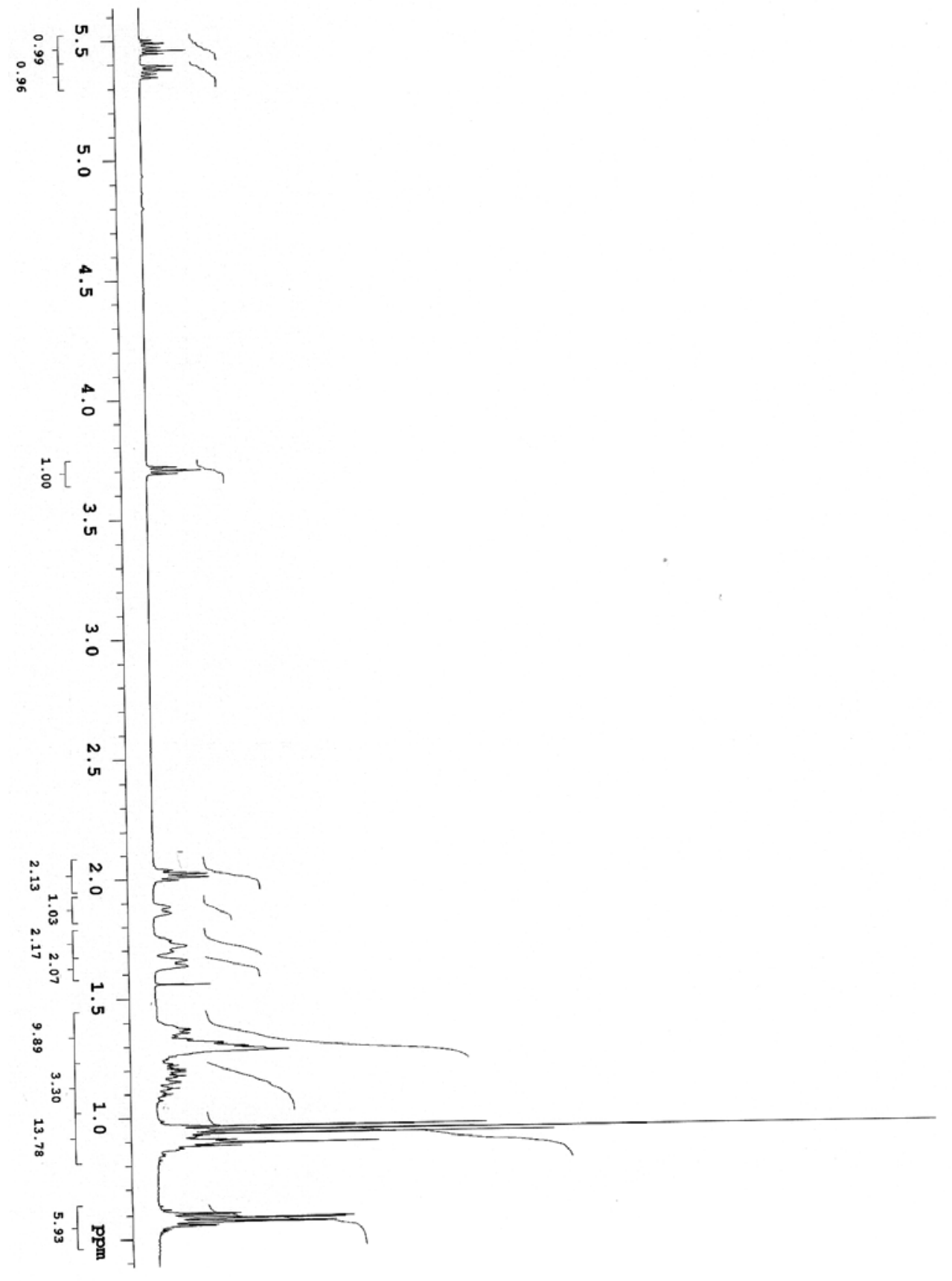




\section{(S,E)-(1-cyclohexylnon-2-enyloxy)triethylsilane (entry 8)}

${ }^{19}$ F-NMR of $R$-Mosher ester

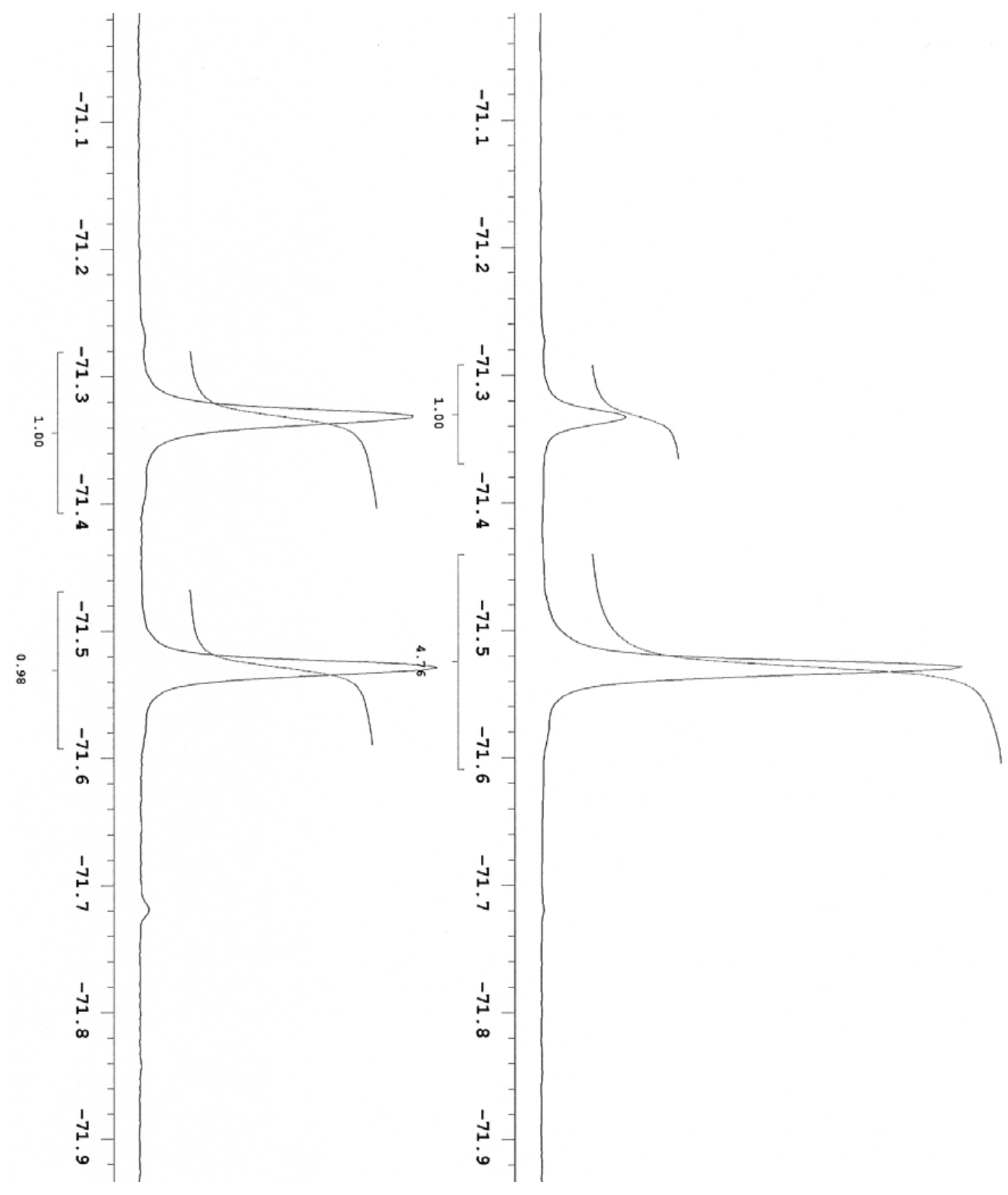


(S,E)-triethyl(2-methyl-1-phenylnon-1-en-3-yloxy)silane (entry 9)

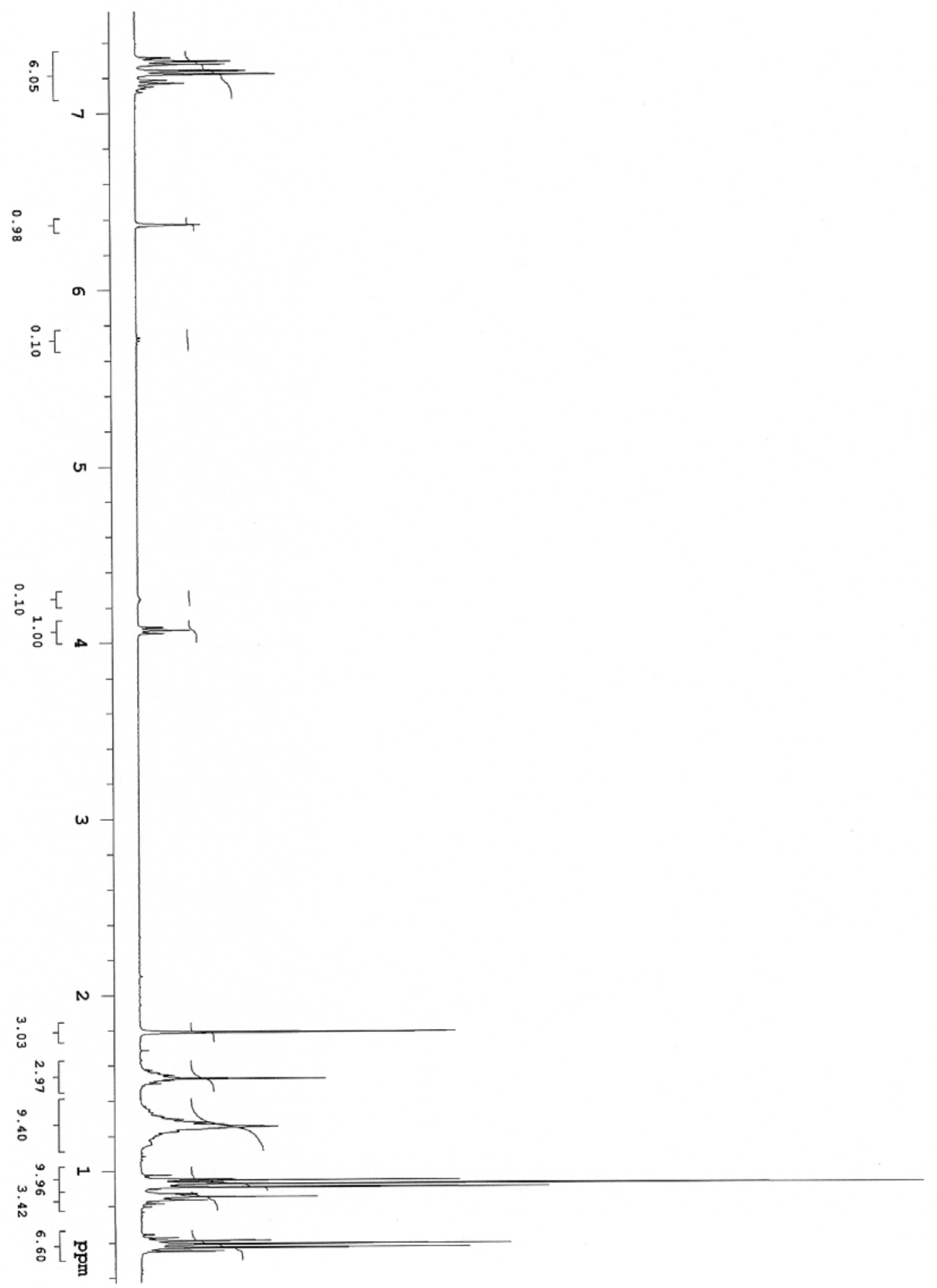




\section{(S,E)-triethyl(2-methyl-1-phenylnon-1-en-3-yloxy)silane (entry 9)}

${ }^{1} \mathrm{H}-\mathrm{NMR}$ of $R$-Mosher ester

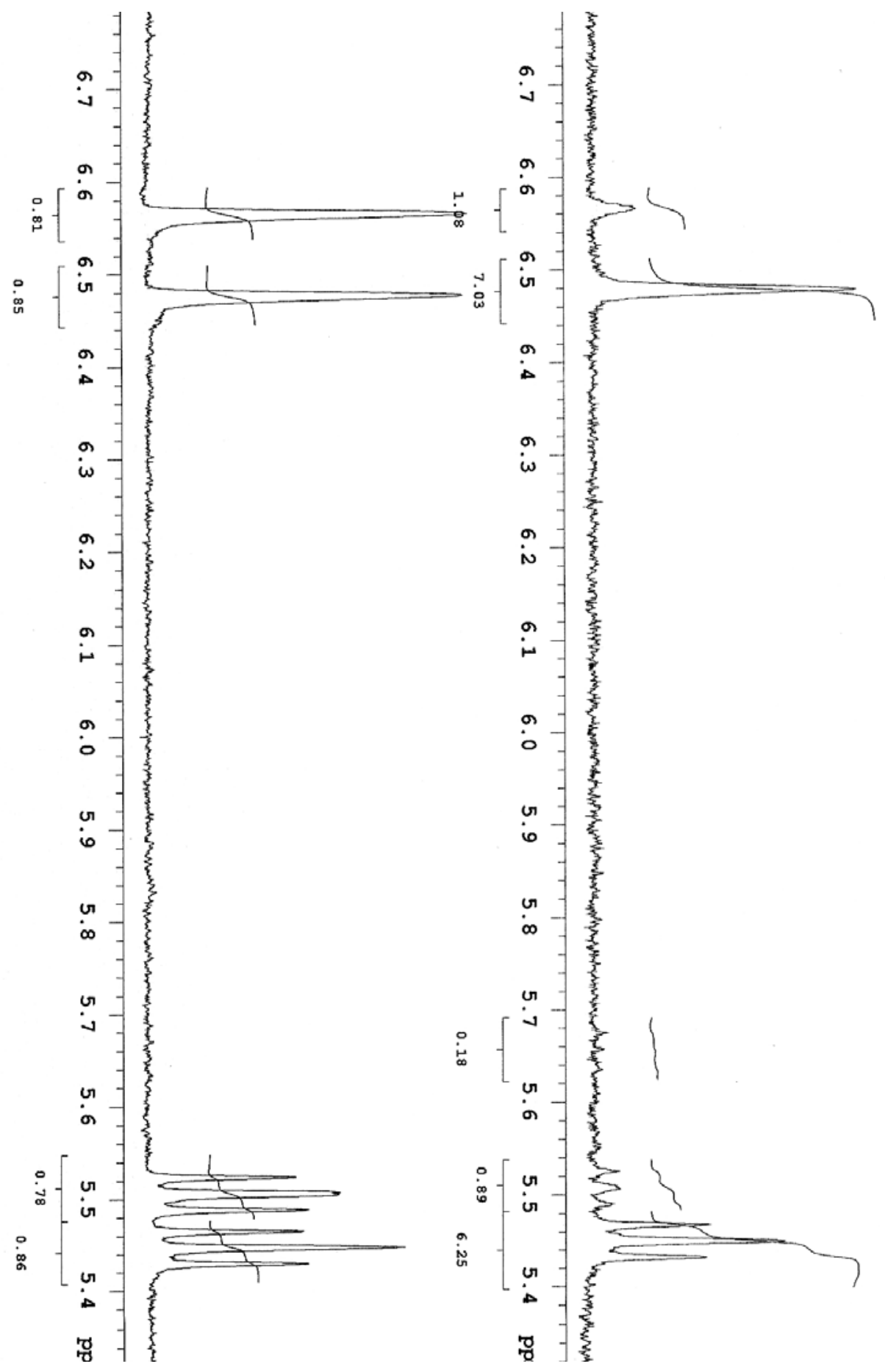


(S,E)-5-(cyclohexyl(triethylsiloxy)methylhex-5-en-1-ol (entry 10)

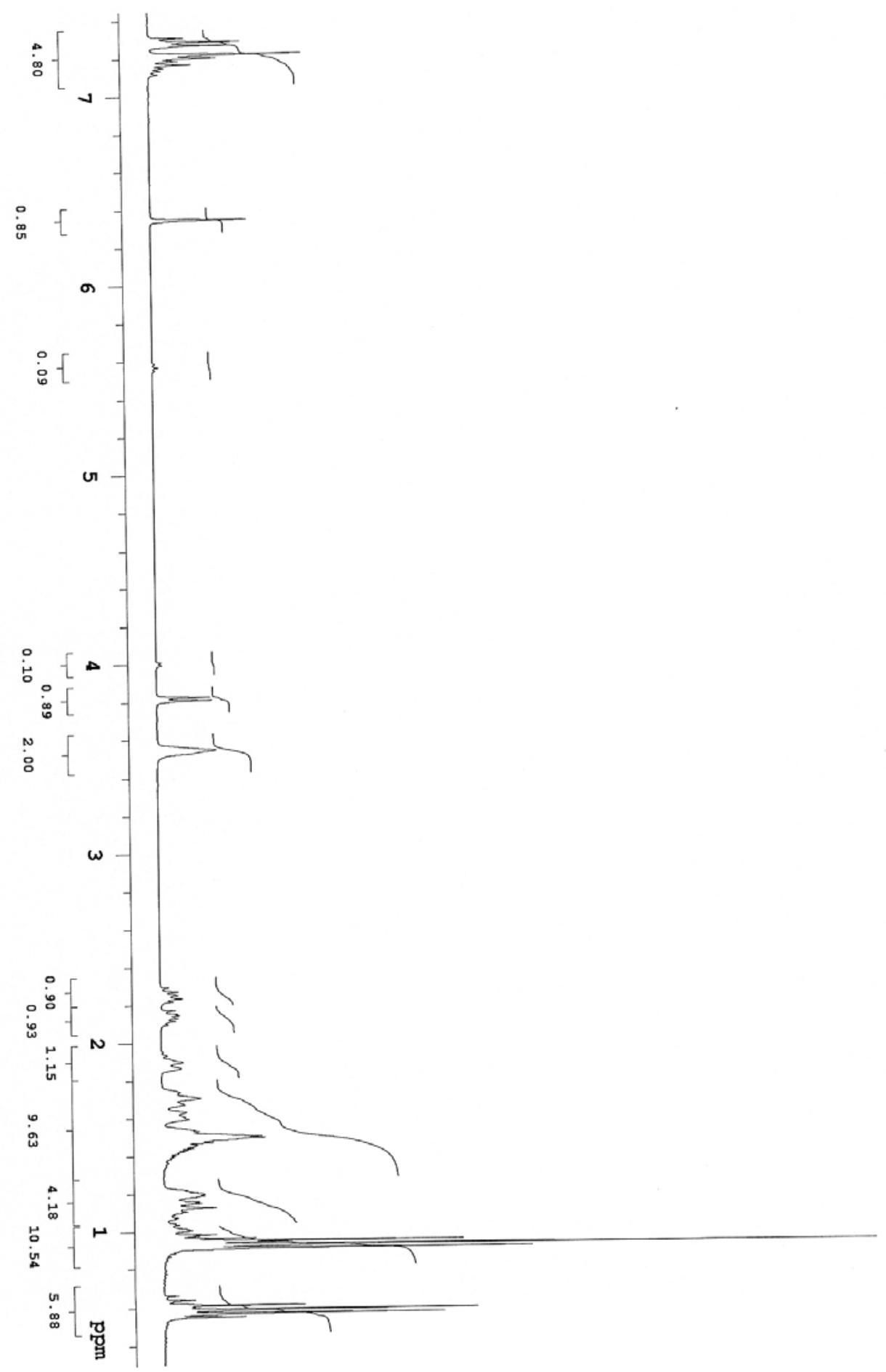




\section{(S,E)-5-(cyclohexyl(triethylsiloxy)methylhex-5-en-1-ol (entry 10)}

${ }^{1} \mathrm{H}$-NMR of $R$-Mosher ester
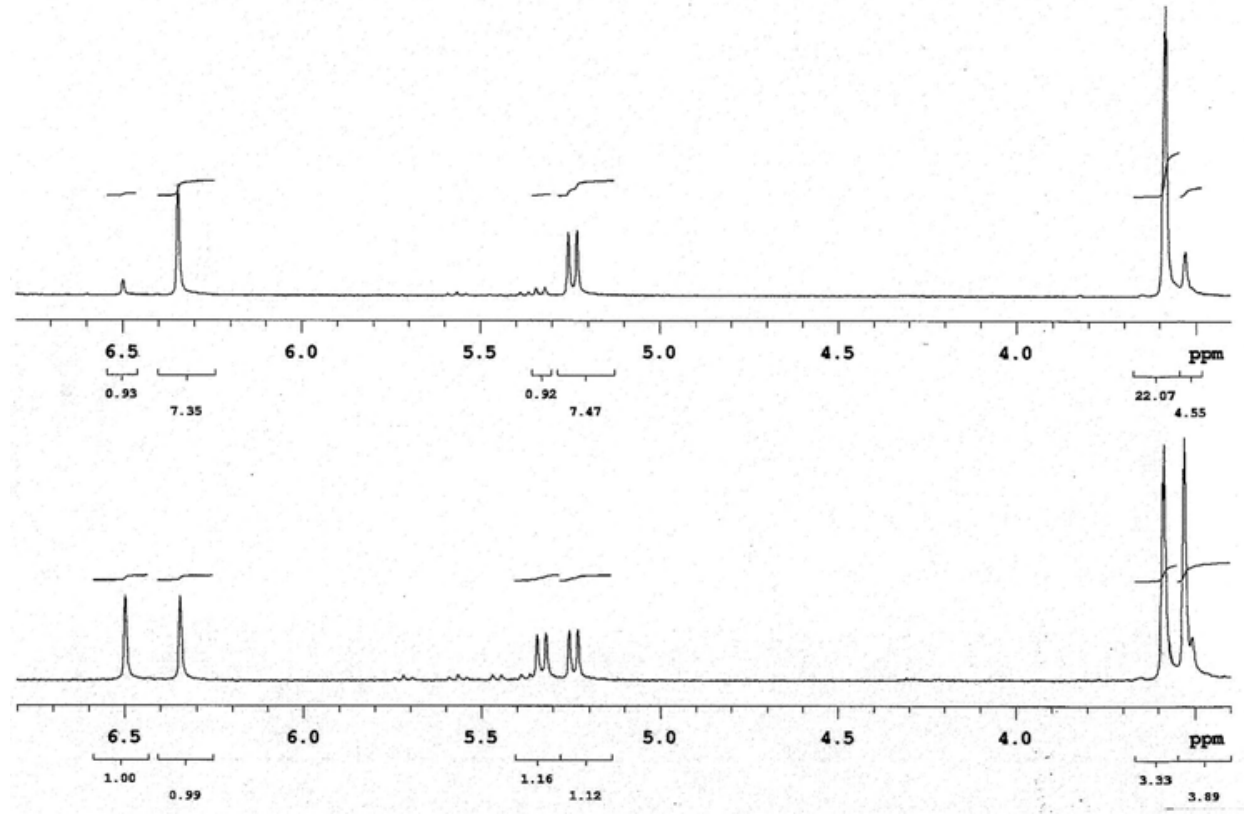

${ }^{19} \mathrm{~F}$-NMR of $R$-Mosher ester
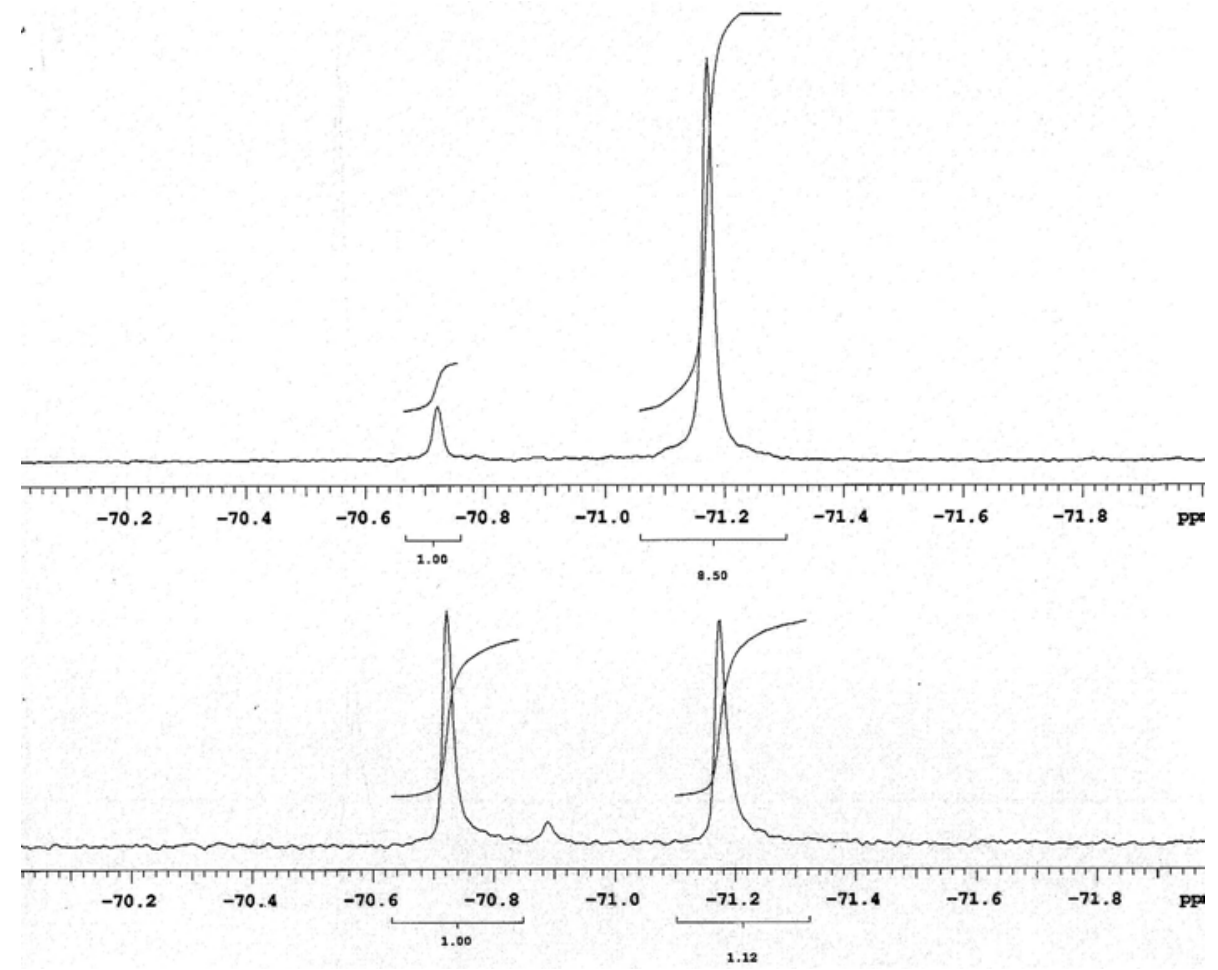
S,E)-(1-cyclohexyl-2-ethylideneheptyloxy)triethylsilane (entry 11)

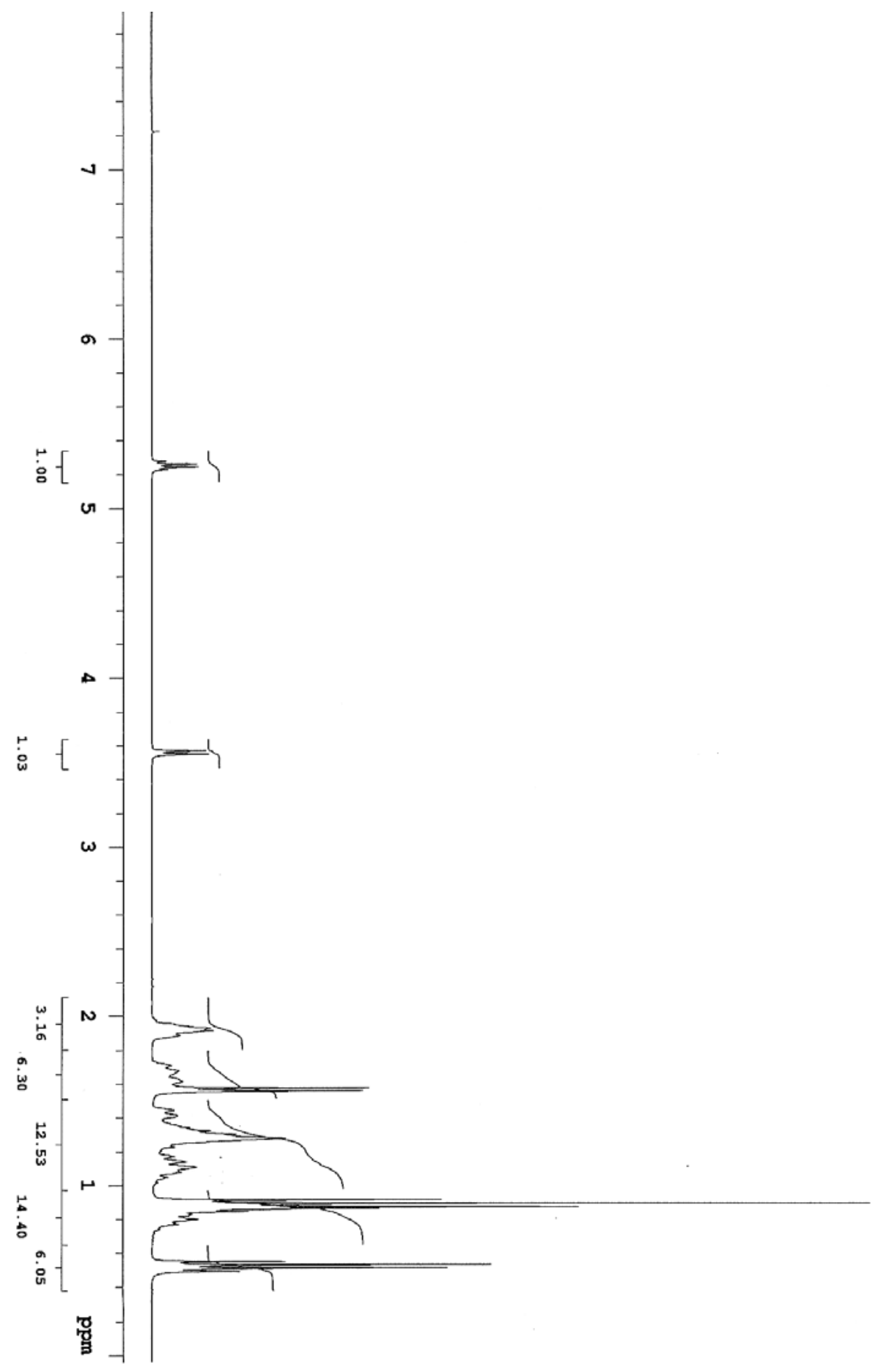




\section{S,E)-(1-cyclohexyl-2-ethylideneheptyloxy)triethylsilane (entry 11)}

${ }^{1} \mathrm{H}-\mathrm{NMR}$ of $R$-Mosher ester

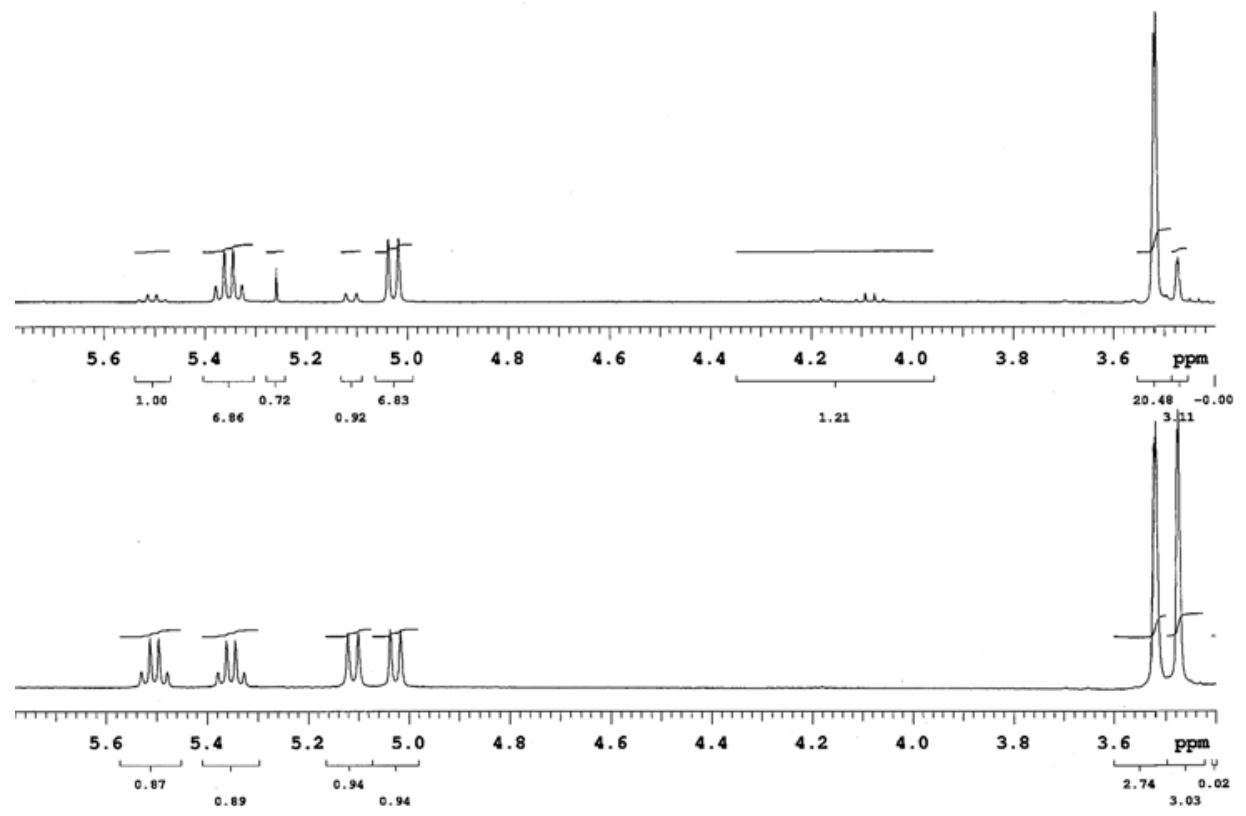

${ }^{19}$ F-NMR of $R$-Mosher ester

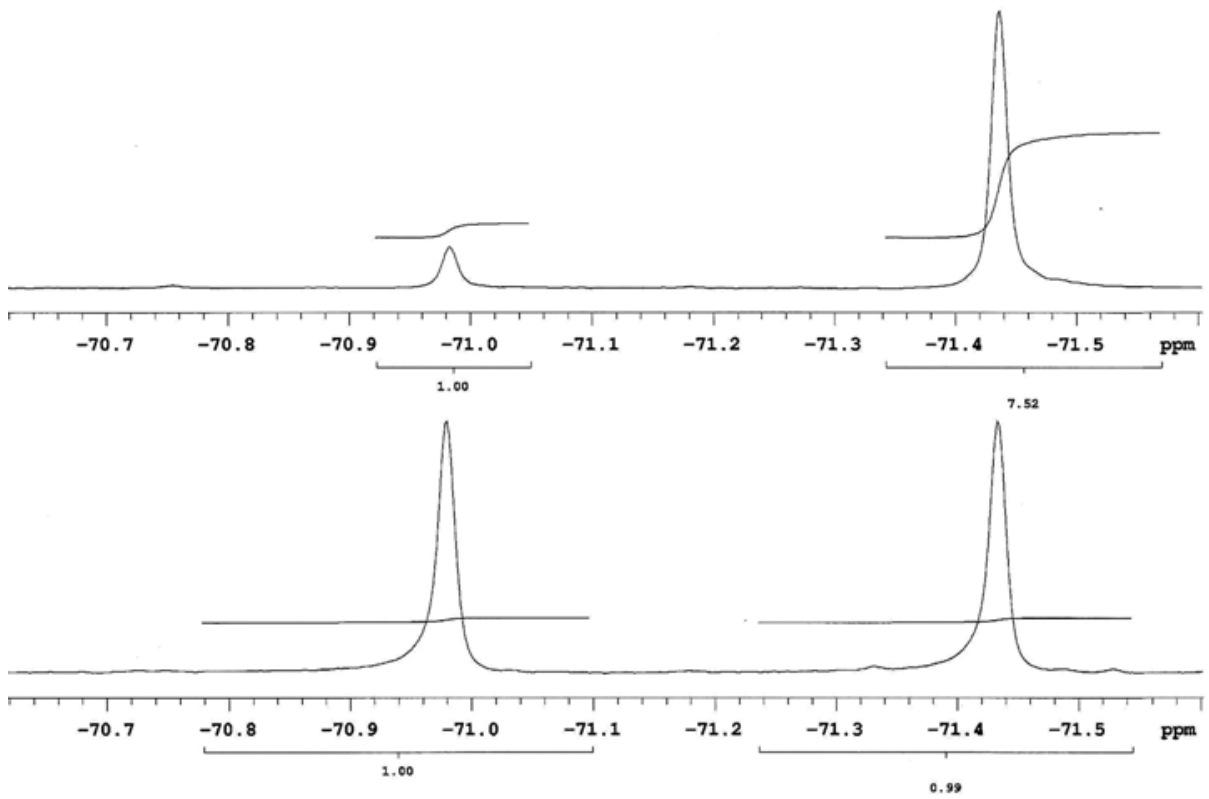




\section{(S,E)-(1-cyclohexyl-2-methyloct-2-enyloxy)triethylsilane (entry 12)}

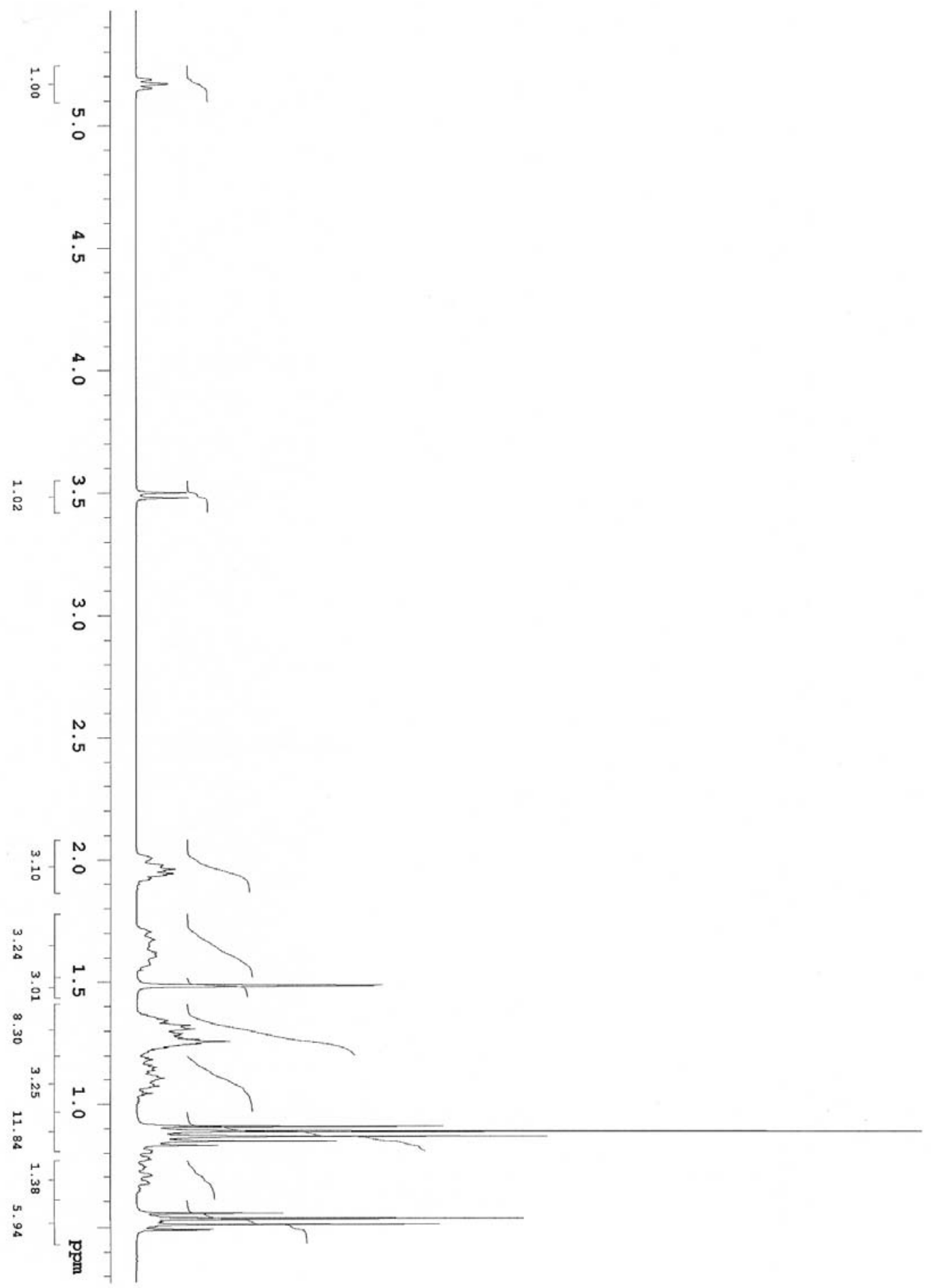




\section{(S,E)-(1-cyclohexyl-2-methyloct-2-enyloxy)triethylsilane (entry 12)}

${ }^{1} \mathrm{H}-\mathrm{NMR}$ of $R$-Mosher ester

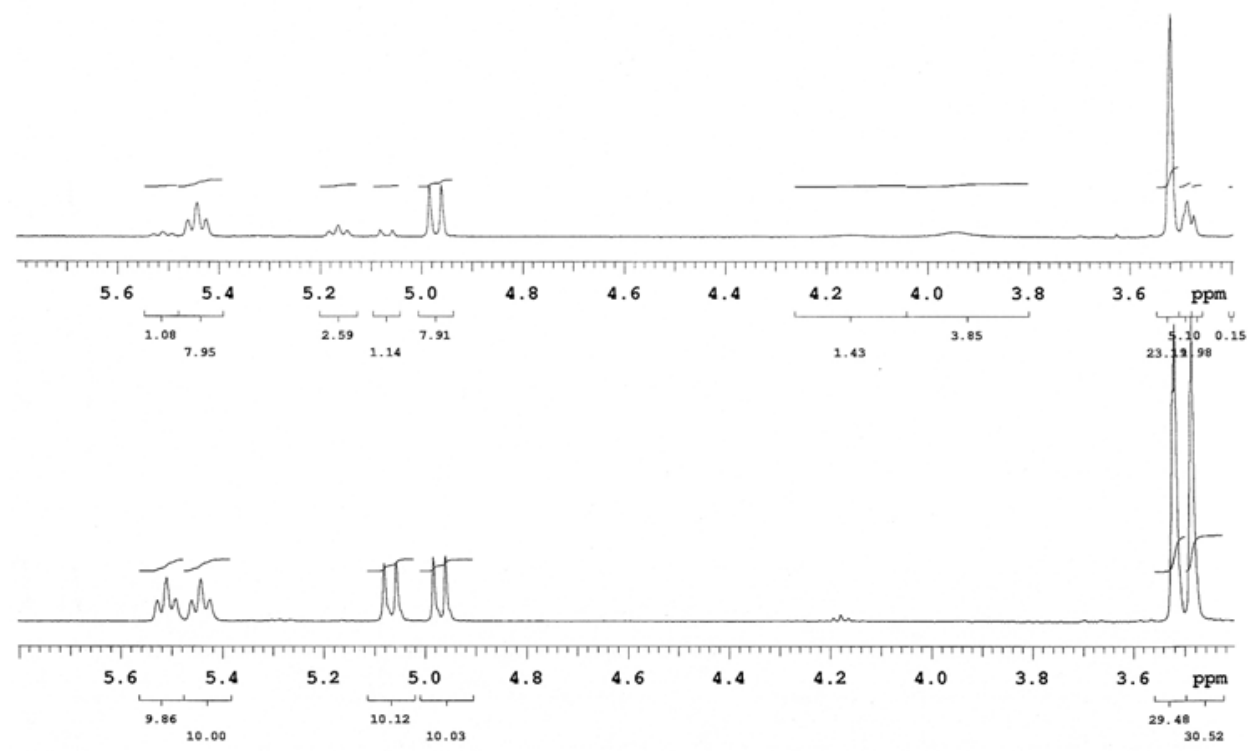

${ }^{19}$ F-NMR of $R$-Mosher ester

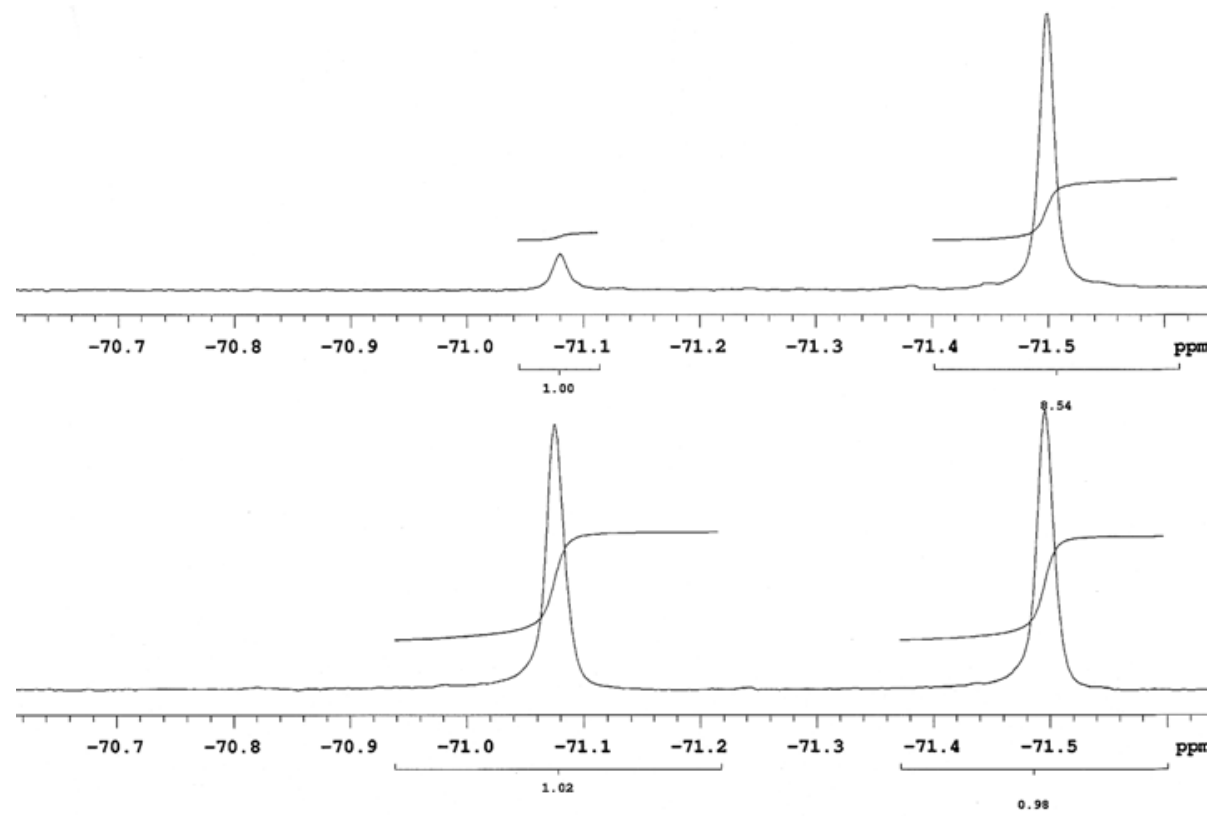


$(S, E)$-triethyl(2-methylcyclotetradec-2-enyloxy)silane and regioisomer (4a and 4b)

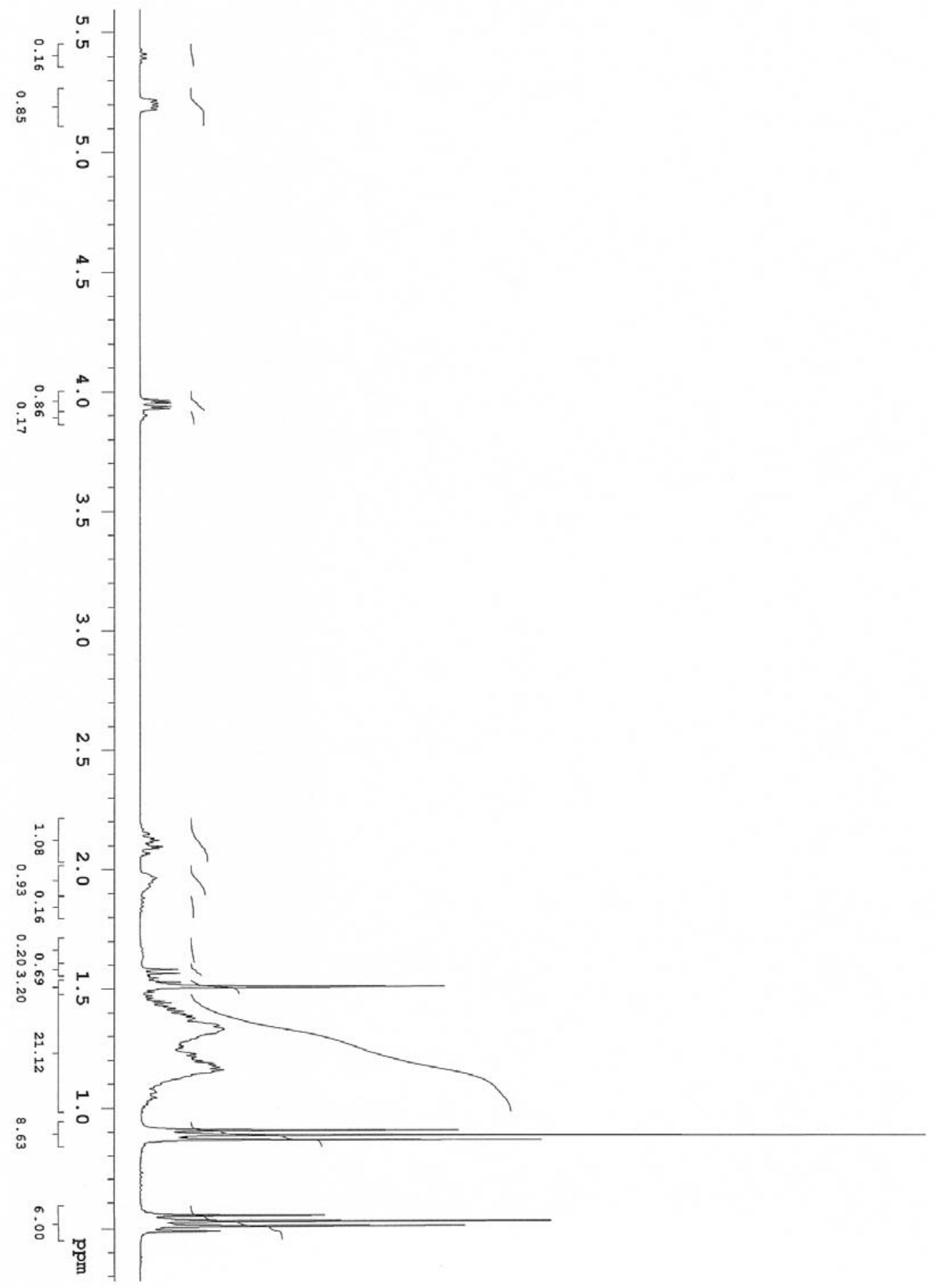




\section{(S,E)-triethyl(2-methylcyclotetradec-2-enyloxy)silane (4a)}

${ }^{1} \mathrm{H}-\mathrm{NMR}$ of $R$-Mosher ester

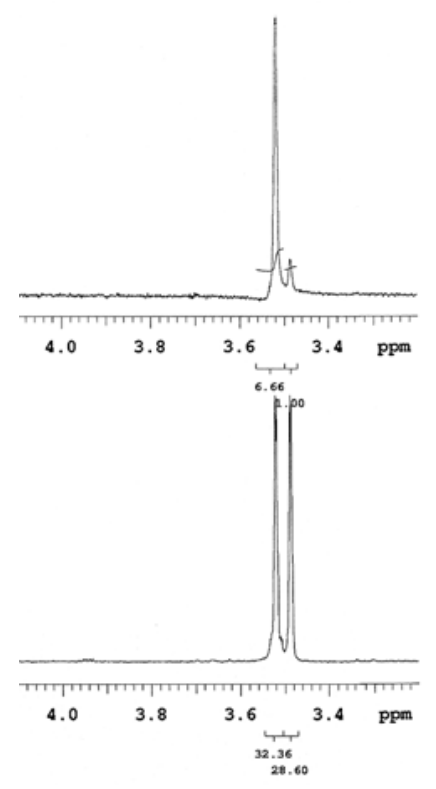

${ }^{19}$ F-NMR of $R$-Mosher ester

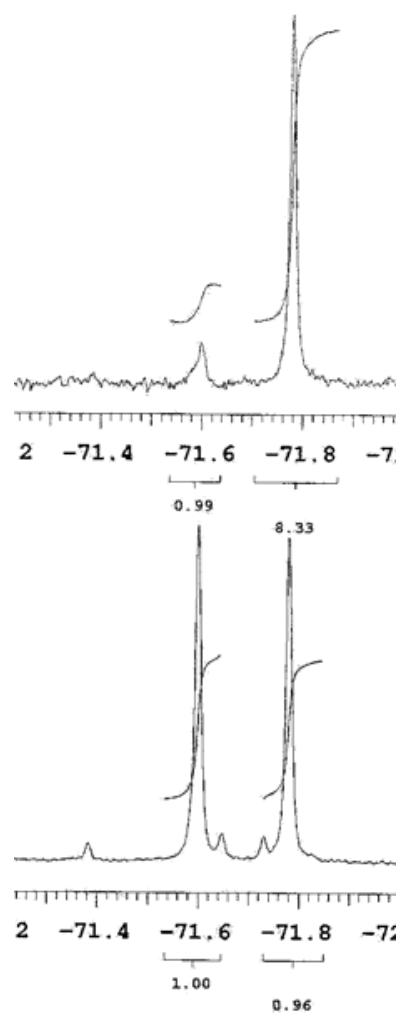


4a $\left({ }^{1} \mathrm{H}\right.$ NMR of $R$-Mosher Ester)

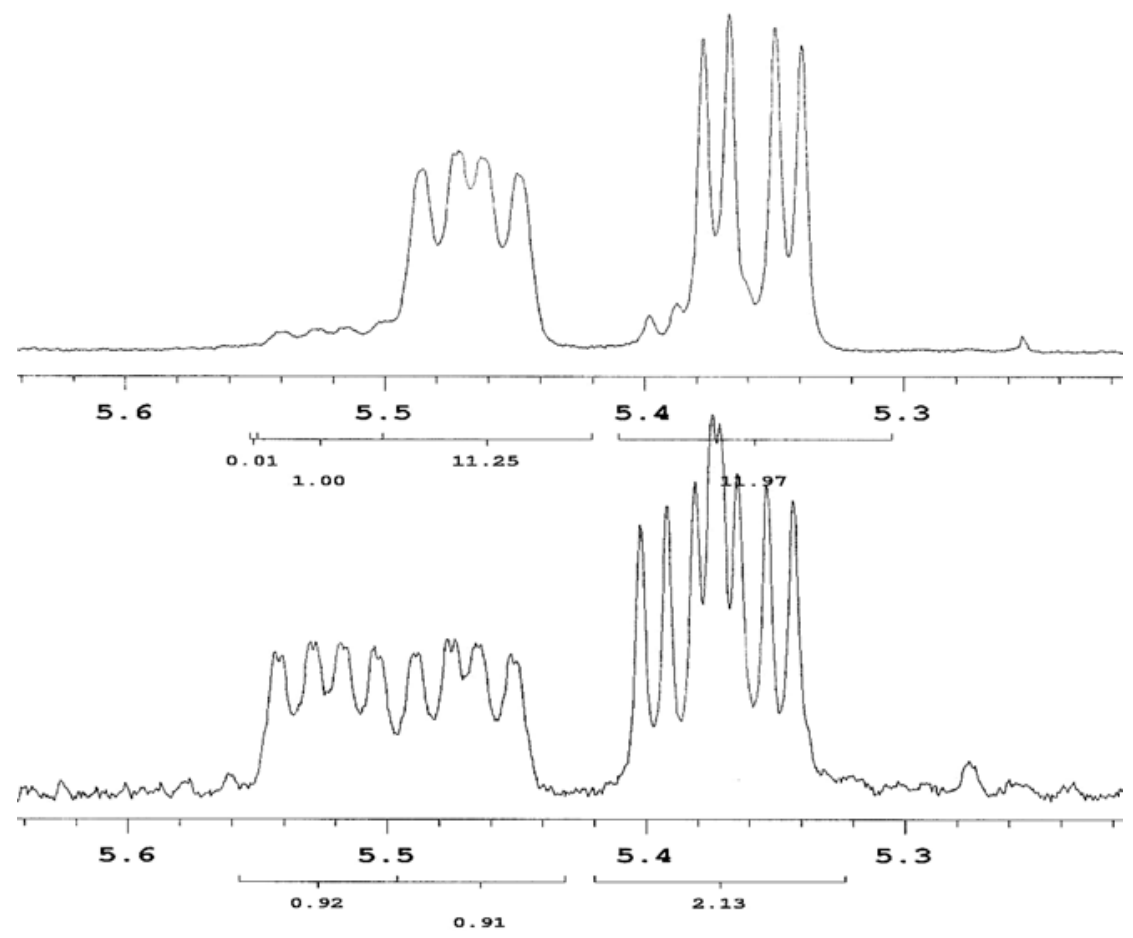

4b (Partially separated from 4a) $\left({ }^{1} \mathrm{H}\right.$ NMR of $R$-Mosher Ester)
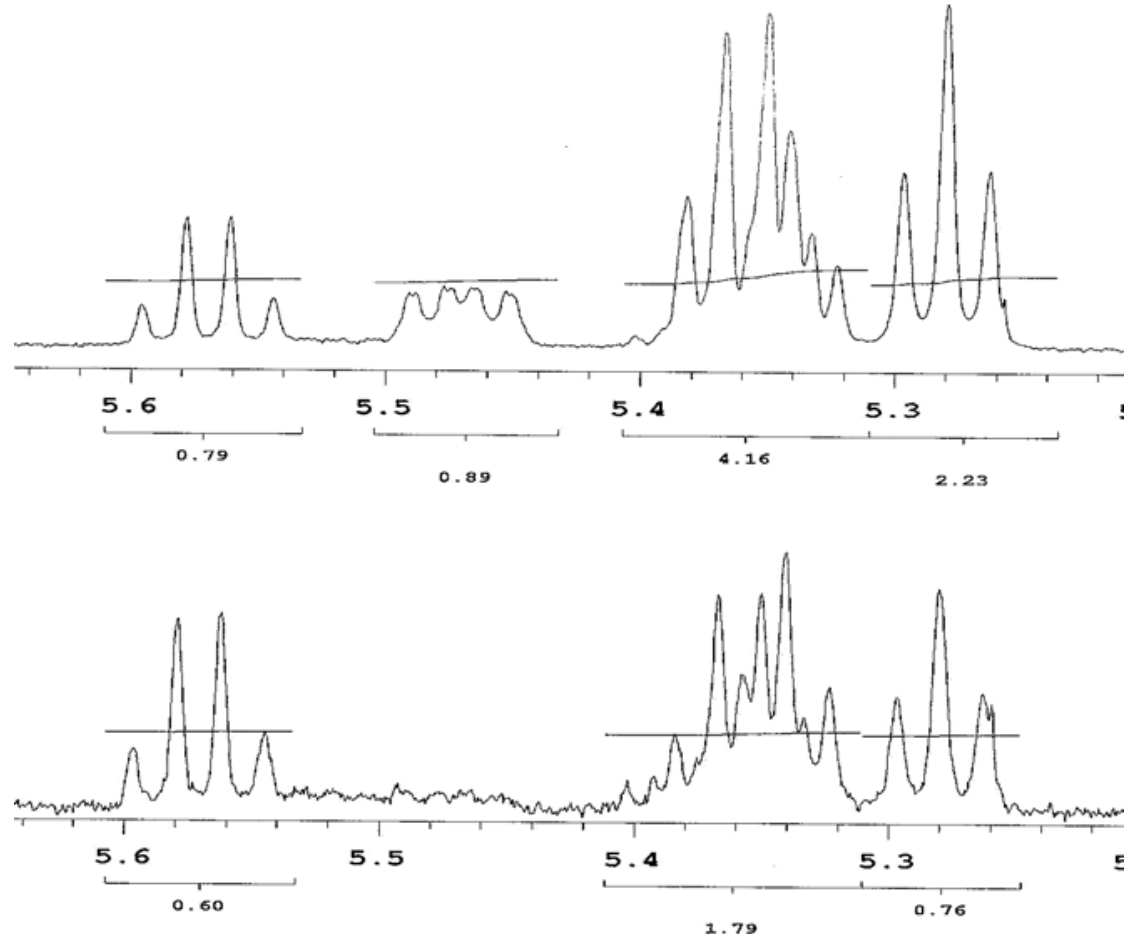